JOURNAL OF

SYMPLECTIC GEOMETRY

Volume 3, Number 4, 481-530, 2005

\title{
ENLARGING THE HAMILTONIAN GROUP ${ }^{\dagger}$
}

\author{
Dusa McDufF
}

This paper investigates ways to enlarge the Hamiltonian subgroup Ham of the symplectomorphism group Symp of a symplectic manifold $(M, \omega)$ to a group that both intersects every connected component of Symp and characterizes symplectic bundles with fiber $\boldsymbol{M}$ and closed connection form. As a consequence, it is shown that bundles with closed connection form are stable under appropriate small perturbations of the symplectic form. Further, the manifold $(\boldsymbol{M}, \boldsymbol{\omega})$ has the property that every symplectic $\boldsymbol{M}$-bundle has a closed connection form if and only if the flux group vanishes and the flux homomorphism extends to a crossed homomorphism defined on the whole group Symp. The latter condition is equivalent to saying that a connected component of the commutator subgroup [Symp, Symp] intersects the identity component of Symp only if it also intersects Ham. It is not yet clear when this condition is satisfied. We show that if the symplectic form vanishes on 2-tori, the flux homomorphism extends to the subgroup of Symp acting trivially on $\boldsymbol{\pi}_{\mathbf{1}}(\boldsymbol{M})$. We also give an explicit formula for the KotschickMorita extension of the flux homomorphism in the monotone case. The results in this paper belong to the realm of soft symplectic topology, but raise some questions that may need hard methods to answer.

\section{Contents}

\section{Introduction}

1.1. Statement of the problem 482

1.2. Extending the fiberwise symplectic form 484

1.3. Extending the flux homomorphism 487

Mathematics Subject Classification. 53C15, 53D35, 57R17.

${ }^{\dagger}$ This paper was first submitted before I saw Kȩdra et al. [6], although most of their paper was completed earlier than mine. In this revision, I have added a few remarks to clarify the relation of their work to mine. I have also reworked some arguments using insights from Gal-Kẹdra [3]. 
1.4. Further results and remarks

2. Definition and Properties of $\widehat{F}_{s} \quad 496$

3. Bundles with structural group $\mathrm{Ham}^{\mathrm{s}} \quad 502$

3.1. Groups with the extension property 502

3.2. The obstruction class 504

3.3. Stability 510

4. Further considerations $\quad 512$

4.1. The integral case 513

4.2. Lifting $\widehat{F}_{s} \quad 517$

4.3. The atoroidal case $\quad 523$

4.4. $c$-Hamiltonian bundles and covering groups $\quad 526$

$\begin{array}{ll}\text { References } & 529\end{array}$

\section{Introduction}

1.1. Statement of the problem. This paper studies what might be called the gross algebro-topological structure of the symplectomorphism group $\operatorname{Symp}(M, \omega)$ of a closed connected symplectic manifold $(M, \omega)$. It is very well known that the identity component $\operatorname{Symp}_{0}(M, \omega)$ of this group supports a continuous homomorphism called Flux that takes values in the quotient $H^{1}(M ; \mathbb{R}) / \Gamma$ of the first de Rham cohomology group with the flux group $\Gamma$. (Precise definitions are given in Section 1.3 below. By recent work of Ono [17], the group $\Gamma$, which is the image of $\pi_{1}$ (Symp) under the flux, is a discrete subgroup of $H^{1}(M ; \mathbb{R})$.)

The kernel of Flux is the Hamiltonian group $\operatorname{Ham}(M, \omega)$, whose elements are the time-1 maps of time-dependent Hamiltonian flows. Since $\operatorname{Ham}(M, \omega)$ is perfect [1], it supports no nontrivial homomorphisms. Hence the only proper normal subgroups of $\mathrm{Symp}_{0}$ lie between Ham and $\mathrm{Symp}_{0}$. One of the motivations for this paper is to understand the extent to which this structure in the identity component of Symp extends to the full group. What interesting normal (or approximately so) subgroups does Symp contain? We shall tacitly assume that $H^{1}(M ; \mathbb{R}) \neq 0$, since otherwise the whole discussion becomes trivial.

One can look at this question either algebraically or topologically. From an algebraic point of view, the most important feature here is the flux homomorphism. Kotschick and Morita pointed out in [9] that although Flux does not in general extend to a homomorphism defined on Symp, it does extend to a crossed homomorphism at least when $(M, \omega)$ is monotone, for example a Riemann surface of genus $>1$. Therefore, one interesting question is:

does Flux always extend to a crossed homomorphism from Symp to $H^{1}(M ; \mathbb{R}) / \Gamma$ ? 
So far, this is unresolved. However, Proposition 1.13 gives equivalent geometric conditions for such an extension to exist.

Kotschick and Morita's proof that Flux does extend in the monotone case is indirect. We explain it at the beginning of Section 4.2. Theorem 4.6 below gives an explicit construction for the extension, which is based on looking at lifts of the action of the elements of Symp to an appropriate line bundle over the universal cover of $M$. Much of our work was motivated by the search for similar explicit formulas. For example, we show in Section 2 that the composite of Flux : $\operatorname{Symp}_{0} \rightarrow H^{1}(M ; \mathbb{R}) / \Gamma$ with a suitable projection $H^{1}(M ; \mathbb{R}) / \Gamma \rightarrow H^{1}(M ; \mathbb{R}) / \mathcal{P}_{\omega}$ always extends.

From a topological/geometric point of view, it turns out that these questions are very closely connected to properties of locally trivial symplectic $M$-bundles $(M, \omega) \rightarrow P \rightarrow B$. A basic problem here is:

to understand when the fiberwise symplectic class $a:=[\omega]$ extends to a class $\widetilde{a} \in H^{*}(P ; \mathbb{R})$.

For example, if $B$ is simply connected, $\widetilde{a}$ exists if and only if the structural group of the bundle reduces to Ham. One reason to be interested in such extensions is that the class $\widetilde{a}$ may be used to define characteristic classes that carry interesting information: see Kȩdra-McDuff [7] and KotschickMorita [10]. Another is that by work of Lalonde et al. $[\mathbf{1 1}, \mathbf{1 2}]$, bundles with structural group Ham have interesting cohomological properties. For instance, for many bases $B$, the rational cohomology of $P$ splits as the tensor product of the cohomology of $B$ with that of $M$.

One would like to know to what extent such results remain true for more general symplectic bundles. More precisely, for which subgroups $\mathcal{H}$ of Symp could one hope to extend the above results to bundles with structural group $\mathcal{H}$ ? Thus, another motivating problem is:

to define a subgroup $\mathcal{H}$ of Symp with the property that the structural group of $P \rightarrow B$ reduces to $\mathcal{H}$ exactly when a extends to $\widetilde{a}$.

Such a group $\mathcal{H}$ can be thought of as an enlargement of Ham - whence the title of this paper. We shall see that suitable groups $\mathcal{H}$ always exist; in Section 2, we construct such $\mathcal{H}$ as kernels of extensions of homomorphisms related to the flux. Proposition 1.15 shows that the stability properties of the Hamiltonian group that were proved in $[\mathbf{1 2}]$ extend to $\mathcal{H}$.

The next question is to understand the obstruction to the reduction of the structural group to $\mathcal{H}$; equivalently, when does the classifying map $B \rightarrow B$ Symp of the bundle $P \rightarrow B$ lift to $B \mathcal{H}$ ? If the Flux group $\Gamma$ vanishes, then, as was pointed out in Kędra et al. [6], Kotschick and Morita's argument may be used to show that this obstruction vanishes if and only if the Flux homomorphism extends. Theorem 1.10 gives the general result, which holds even when $\Gamma \neq 0$. 
In the rest of this introduction, we discuss each of the main questions above in more detail. In Section 1.4, we explain some examples and also (in Remark 1.20) some of the special features that arise when $[\omega]$ is an integral class.

1.2. Extending the fiberwise symplectic form. Let $(M, \omega)$ be a closed connected symplectic manifold, and consider a locally trivial symplectic $M$-bundle

$$
(M, \omega) \rightarrow P \rightarrow B
$$

over a connected base $B$. We want to understand when the fiberwise symplectic class $a:=[\omega]$ extends to a class $\widetilde{a} \in H^{*}(P ; \mathbb{R})$. By Thurston's construction, this is equivalent to saying that the family of fiberwise symplectic forms $\omega_{b}, b \in B$, has a closed extension $\Omega$ to $P$. (Here we assume without loss of generality that $P \rightarrow B$ is smooth.) For short, we will often call the family $\omega_{b}$ simply the fiberwise symplectic form.

This topic was first studied by Gotay et al. [4] where they showed that each extension $\Omega$ of the fiberwise symplectic form gives rise to an Ehresmann connection on the bundle $P \rightarrow B$ whose horizontal spaces are the $\Omega$-orthogonals to the fibers. This connection has symplectic holonomy iff the restriction of $\Omega$ over the preimages of arcs in the base is closed and it has Hamiltonian holonomy round all contractible loops iff $\Omega$ is closed. Therefore, we shall call closed extensions of the fiberwise form closed connection forms.

If $H^{1}(M ; \mathbb{R})=0$, then the Guillemin-Lerman-Sternberg (GLS) construction provides a closed connection form on every symplectic bundle. In the general case, we are looking for a group homomorphism $\mathcal{H} \rightarrow \operatorname{Symp}(M, \omega)$ such that an $M$-bundle $P \rightarrow B$ over a connected finite simplicial complex has a closed connection form iff its classifying map $\phi: B \rightarrow B$ Symp lifts to $B \mathcal{H}$. For short, we shall say that such a homomorphism $\mathcal{H} \rightarrow \operatorname{Symp}(M, \omega)$ (or simply the group $\mathcal{H}$ ) has the extension property. In particular, the group Symp itself has the extension property iff every symplectic $M$-bundle has a closed connection form.

The GLS construction also implies that a symplectic bundle $P \rightarrow B$ over a simply connected base $B$ has a closed connection form if and only if its structural group can be reduced to the Hamiltonian group $\operatorname{Ham}(M, \omega)$. These fibrations are classified by maps $\phi: B \rightarrow B$ Symp $_{0}$ (where Symp $_{0}$ denotes the identity component of the group Symp), and in this restricted case we may take $\mathcal{H} \rightarrow \operatorname{Symp}_{0}(M, \omega)$ to be the inclusion Ham $\hookrightarrow \operatorname{Symp}_{0}$. Hence the desired group $\mathcal{H}$ should be understood as a generalization of the Hamiltonian group.

There are several natural candidates for $\mathcal{H}$. Perhaps the most elegant approach is due to Seidel [20], who considers a second topology on the symplectomorphism group called the Hamiltonian topology with basis 
consisting of the sets $g U$, for $g \in$ Symp and $U$ open in Ham. We write Symp $^{\text {Htop }}$ for the symplectomorphism group in this topology, reserving Symp to denote the same group but with its usual $C^{\infty}$-topology. The inclusion

$$
\text { Symp }{ }^{\text {Htop }} \rightarrow \text { Symp }
$$

is obviously continuous, but is not a homeomorphism when $H^{1}(M ; \mathbb{R}) \neq 0$. In particular, the identity component of Symp ${ }^{\text {Htop }}$ is the Hamiltonian group, not $\mathrm{Symp}_{0}$. The following result is implicit in [20] and holds by an easy application of the GLS construction: see Section 3.

Proposition 1.1. The inclusion $\mathrm{Symp} \mathrm{H}^{\mathrm{Htop}} \rightarrow \mathrm{Symp}$ has the extension property, i.e., a symplectic $M$-bundle has a closed connection form iff its classifying map lifts to $B$ Symp ${ }^{\text {Htop }}$.

The group Symp ${ }^{\text {Htop }}$ is natural but very large. For example, its intersection with $\mathrm{Symp}_{0}$ has uncountably many components when $H^{1}(M ; \mathbb{R}) \neq 0$. We define in Section 1.3 below a closed subgroup

$$
\mathrm{Ham}^{s}
$$

of Symp ${ }^{\text {Htop }}$ that still has the extension property, but has the homotopy type of a countable CW complex. ${ }^{1}$ Another advantage of this group is that it has an algebraic (rather than topological) relation to Symp, which makes it easier to understand the homotopy fiber of the induced map $B \mathrm{Ham}^{s} \rightarrow$ $B$ Symp.

The group $\mathrm{Ham}^{s}$ is a union of connected components of Symp ${ }^{\mathrm{Htop}}$. It intersects every component of Symp, and when $H^{1}(M ; \mathbb{R}) \neq 0$ intersects Symp $_{0}$ in a countably infinite number of components. Hence in general, this subgroup is not closed in Symp. However, as we point out in Remark 1.9, if $\Gamma \neq 0$, no closed subgroup of Symp can have the extension property.

One reason for this is that Ham (which is closed in Symp by Ono [17]) does not quite classify the set of Symp ${ }_{0}$-bundles for which $a$ extends. To explain this, it is useful to introduce the following definitions. We suppose as before that $M \rightarrow P \rightarrow B$ is a smooth bundle with base equal to a finite dimensional (possibly open) connected manifold with finite homotopy type and fiber a closed symplectic manifold. Recall also that a symplectic bundle has classifying map $\phi: B \rightarrow B$ Symp $_{0}$ iff it is symplectically trivial over the 1-skeleton $B_{1}$ of the base.

Definition 1.2. We shall say that a (possibly disconnected) subgroup $\mathcal{H}_{0}$ of Symp $_{0}$ has the restricted extension property if the following condition holds: a Symp $_{0}$-bundle $M \rightarrow P \rightarrow B$ has a closed connection form iff its classifying map $B \rightarrow B$ Symp $_{0}$ lifts to $B \mathcal{H}_{0}$. Similarly, a subgroup $\mathcal{H}$ of Symp has the modified extension property if the following condition

\footnotetext{
${ }^{1} \mathrm{~A}$ proof that Symp and Ham have the homotopy type of a countable CW complex is sketched in McDuff-Salamon [16, 9.5.6].
} 
holds: a Symp-bundle $M \rightarrow P \rightarrow B$ has a closed connection form iff the pullback of its classifying map $B \rightarrow B$ Symp over some finite cover $\rho: \widetilde{B} \rightarrow$ $B$ lifts to $B \mathcal{H}$.

Thus, to say that $\mathcal{H}_{0}$ has the modified restricted extension property means that a Symp $_{0}$-bundle $M \rightarrow P \rightarrow B$ has a closed connection form iff there is a homotopy commutative diagram

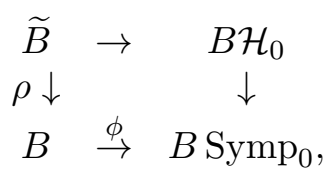

where $\rho: \widetilde{B} \rightarrow B$ is some finite covering map and $\phi: B \rightarrow B$ Symp $_{0}$ classifies $P \rightarrow B$.

One aim of this paper is to understand subgroups $\mathcal{H}$ of Symp that have the (possibly modified) extension property. Since every $M$-bundle $P \rightarrow S^{1}$ has a closed connection form, any such group $\mathcal{H}$ must intersect almost every component of Symp. The following proposition is proved in Section 3.2. We write $\operatorname{Im}\left(\pi_{0}(\mathcal{H})\right)$ for the image of $\pi_{0}(\mathcal{H})$ in $\pi_{0}(\operatorname{Symp})$.

Proposition 1.3. Let $\mathcal{H}$ be a subgroup of Symp with identity component equal to Ham. Then $\mathcal{H}$ has the modified extension property iff every finitely generated subgroup of $\pi_{0}(\mathrm{Symp})$ has finite image in the coset space $\pi_{0}(\operatorname{Symp}) / \operatorname{Im}\left(\pi_{0}(\mathcal{H})\right)$.

Lalonde-McDuff [11] and early reprints of McDuff-Salamon [15, Theorem 6.36] claim that the Hamiltonian group $\operatorname{Ham}(M, \omega)$ has the restricted extension property. But this is false: there are $\operatorname{Symp}_{0}$-bundles $P \rightarrow B$ that have a closed connection form but yet only acquire a Hamiltonian structure when pulled back over some finite covering $\widetilde{B} \rightarrow B$. (See later reprints of $[\mathbf{1 5}]$ and the erratum to [11].) The next proposition is proved in Section 3.2.

Proposition 1.4. The Hamiltonian group $\operatorname{Ham}(M, \omega)$ has the modified restricted extension property. It has the restricted extension property iff $\Gamma=0$.

The previous results prompt the following question.

Question 1.5. When does Symp have a subgroup $\mathcal{H}$ with the modified extension property and such that $\mathcal{H} \cap \mathrm{Symp}_{0}=\mathrm{Ham}$ ?

As is shown by Proposition 1.8 below, this is very closely related to questions about extending the Flux homomorphism.

The next problem is to understand the obstruction to the existence of a closed connection form. The following lemma is proved in Kȩdra-McDuff [7].

Lemma 1.6. A symplectic $M$-bundle $\pi: P \rightarrow B$ has a closed connection form iff the restriction of $\pi$ over the 2-skeleton of $B$ has such a form. 
This is mildly surprising: in order for the fiberwise symplectic class $[\omega]$ to extend to $H^{2}(P)$, it must lie in the kernel of the Leray-Serre differential $d_{3}$ as well as in ker $d_{2}$, and in principle, $d_{3}$ depends on the 3 -skeleton of $B$. However, Lemma 1.6 is a very general result that is valid in the cohomologically symplectic case, i.e., for pairs $(M, a)$ where $M$ is a closed oriented $2 n$-manifold and $a \in H^{2}(M)$ has $a^{n}>0$. To prove it, observe that if $a$ survives to $E_{3}^{0,2}$, then $d_{3}\left(a^{n+1}\right)=(n+1) d_{3}(a) \otimes a^{n}$ must vanish since $a^{n+1}=0$. But because tensoring with $a^{n}$ gives an isomorphism $E_{3}^{3,0} \rightarrow E_{3}^{3,2 n}$ and the cohomology groups have coefficients $\mathbb{R}$, this is possible only if $d_{3} a=0$.

Although the obstruction lies in such low dimensions, it is still not fully understood. It divides into two parts, one that depends on the "symplectic mapping class group" $\pi_{0}$ (Symp) and the other on the flux subgroup $\Gamma$. This is shown by the next proposition that formulates necessary and sufficient conditions for the obstruction to vanish.

Proposition 1.7. The following conditions are equivalent:

(i) Every symplectic $M$-bundle $P \rightarrow B$ has a closed connection form.

(ii) $\Gamma=0$ and every connected component of the commutator subgroup [Symp, Symp] that intersects Symp $_{0}$ also intersects Ham.

The second condition in (ii) is not yet well understood. We show below that it is equivalent to the existence of a suitable extension of the flux homomorphism; see Proposition 1.13 and Remark 1.14. However, we can prove that Flux extends only under very restrictive circumstances, for example, if $[\omega]$ vanishes on 2 -tori and $\pi_{0}$ (Symp) acts on $\pi_{1}(M)$ by inner automorphisms; see Proposition 1.18.

1.3. Extending the flux homomorphism. The flux is initially defined as a homomorphism from the universal cover $\widetilde{\operatorname{Symp}}_{0}$ of the identity component of the symplectomorphism group to the group $H^{1}(M ; \mathbb{R})$. For each element $\left\{g_{t}\right\} \in \widetilde{\operatorname{Symp}}_{0}$, the value of the class $\widetilde{\text { Flux }}\left(\left\{g_{t}\right\}\right) \in H^{1}(M ; \mathbb{R})$ on the 1-cycle $\gamma$ in $M$ is given by integrating $\omega$ over the 2-chain $(s, t) \mapsto g_{t}(\gamma(s))$. If we define the Flux group $\Gamma$ to be the image of $\pi_{1}(\operatorname{Symp}) \subset \widetilde{\text { Symp }_{0}}$ under $\widetilde{\text { Flux }}$, then $\widetilde{\text { Flux }}$ descends to a homomorphism

$$
\text { Flux : } \operatorname{Symp}_{0}(M, \omega) \rightarrow H^{1}(M ; \mathbb{R}) / \Gamma
$$

that we shall call the flux homomorphism. Its kernel is the Hamiltonian group Ham.

The problem of extending the Flux homomorphism to the whole group Symp arose (with rather different motivation) in the work of KotschickMorita [9] in the case when $M$ is a Riemann surface of genus $g>1$ or, more generally a monotone manifold, i.e., a manifold in which the symplectic class $[\omega]$ is a multiple of the first Chern class. They showed that in this case Flux 
extends to a crossed homomorphism

$$
F_{\mathrm{KM}}: \operatorname{Symp}(M, \omega) \rightarrow H^{1}(M ; \mathbb{R}),
$$

that is, a map $F:=F_{\mathrm{KM}}$ that instead of being a homomorphism, satisfies the identity

$$
F(g h)=F(h)+h^{*} F(g),
$$

where $h^{*}$ denotes the action of $h$ on $H^{1}(M ; \mathbb{R})$ via pullback. ${ }^{2}$

In general, one should look for an extension of Flux with values in $H^{1}(M ; \mathbb{R}) / \Gamma$. So far, it is unknown whether an extension must always exist: see Proposition 1.13. However, the following result shows that this question is very closely related to our earlier considerations.

Proposition 1.8. (i) If

$$
\widetilde{F}: \operatorname{Symp}(M, \omega) \rightarrow H^{1}(M ; \mathbb{R}) / \Gamma
$$

is a continuous crossed homomorphism that extends Flux, its kernel $\mathcal{H}$ intersects every component of Symp and has the modified extension property. Moreover, $\mathcal{H}$ has the extension property iff $\Gamma=0$.

(ii) Conversely, let $\mathcal{H}$ be a subgroup of Symp that intersects $\mathrm{Symp}_{0}$ in Ham and denote by $\operatorname{Symp}_{\mathcal{H}}$ the union of the components of Symp that intersect $\mathcal{H}$. Then there is a crossed homomorphism $\widetilde{F}: \operatorname{Symp}_{\mathcal{H}} \rightarrow H^{1}(M ; \mathbb{R}) / \Gamma$ that extends Flux.

Proof. Given $\widetilde{F}$, let $\mathcal{H}:=\mathcal{H}_{\widetilde{F}}$ be the kernel of $\widetilde{F}$. Then $\mathcal{H} \cap \mathrm{Symp}_{0}=$ Ham. Further given any $g \in$ Symp choose $h \in \operatorname{Symp}_{0}$ such that Flux $h=-\widetilde{F}(g) \in$ $H^{1}(M ; \mathbb{R}) / \Gamma$. Then $g$ is isotopic to the element $g h \in \mathcal{H}$. Hence $\mathcal{H}$ has the modified extension property by Proposition 1.3. If $\Gamma=0$, then the inclusion $B \mathcal{H} \rightarrow B$ Symp is a homotopy equivalence and every bundle has both a closed connection form and an $\mathcal{H}$-structure. If $\Gamma \neq 0$, one can construct bundles that have a closed connection form but no $\mathcal{H}$-structure as in the proof of Proposition 1.4.

To prove (ii), we define $\widetilde{F}$ on $\operatorname{Symp}_{\mathcal{H}}$ as follows: given $g \in \operatorname{Symp}_{\mathcal{H}}$, denote by $\sigma_{g}$ any element in $\mathcal{H}$ that is isotopic to $g$ and set $\widetilde{F}(g):=\operatorname{Flux}\left(\sigma_{g}^{-1} g\right)$. This is independent of the choice of $\sigma_{g}$. Further $\left(\sigma_{g} \sigma_{h}\right)^{-1} \sigma_{g h} \in \mathcal{H} \cap \operatorname{Symp}_{0}=$ Ham. Hence

$\widetilde{F}(g h)=\operatorname{Flux}\left(\left(\sigma_{g h}\right)^{-1} g h\right)=\operatorname{Flux}\left(\sigma_{h}^{-1}\left(\sigma_{g}^{-1} g\right) \sigma_{h}\right) \operatorname{Flux}\left(\sigma_{h}^{-1} h\right)=h^{*} \widetilde{F}(g)+\widetilde{F}(h)$.

Thus $\widetilde{F}$ satisfies (1.1) and so is a crossed homomorphism.

\footnotetext{
${ }^{2}$ This is the natural identity to use for a crossed homomorphism $G \rightarrow \mathcal{A}$ when the group $G$ acts contravariantly on the coefficients $\mathcal{A}$. Note also that when Symp acts nontrivially on $H^{1}(M ; \mathbb{R})$, it is not possible to extend Flux to a group homomorphism: see Remark 2.7.
} 
Remark 1.9. If Flux extends to $\widetilde{F}$ but $\Gamma \neq 0$ then by part (i) of the above proposition the kernel of $\widetilde{F}$ does not have the extension property. On the other hand, the kernel $\mathcal{H}_{\mathbb{Q}}$ of the composite map

$$
\widetilde{F}: \operatorname{Symp} \longrightarrow H^{1}(M ; \mathbb{R}) / \Gamma \longrightarrow H^{1}(M ; \mathbb{R}) /(\Gamma \otimes \mathbb{Q})
$$

does have the extension property by Corollary 3.6. It follows from the proof of Proposition 1.4 that this is the smallest group with this property. Note that it has countably many components in $\mathrm{Symp}_{0}$.

Although Flux may not always have an extension with values in $H^{1}(M ; \mathbb{R}) / \Gamma$, its composite with projection onto a suitable quotient group $H^{1}(M ; \mathbb{R}) / \Lambda$ always can be extended. Below we shall define a continuous crossed homomorphism

$$
\widehat{F}_{s}: \operatorname{Symp}(M, \omega) \longrightarrow H^{1}(M ; \mathbb{R}) / H^{1}\left(M ; \mathcal{P}_{\omega}\right)=: \mathcal{A},
$$

where $\mathcal{P}_{\omega}:=\mathcal{P}_{\omega}^{\mathbb{Q}}$ is the rational period group of $\omega$ (i.e., the values taken by $[\omega]$ on the rational 2-cycles). The map $\widehat{F}_{s}$ depends on the choice of a splitting $s$ of a certain exact sequence. (See the definitions in Section 2. The "topology" on $\mathcal{A}$ is explained in Remark 2.6.) However, its restriction to the identity component $\mathrm{Symp}_{0}$ is independent of this choice and equals the composite

$$
\operatorname{Symp}_{0} \stackrel{\text { Flux }}{\longrightarrow} H^{1}(M ; \mathbb{R}) / \Gamma \longrightarrow H^{1}(M ; \mathbb{R}) / H^{1}\left(M ; \mathcal{P}_{\omega}\right) .
$$

Recall that if a group $G$ acts continuously on an $R$-module $\mathcal{A}$ (for suitable ground ring $R$ ), then the continuous group cohomology ${ }^{3}$

$$
H_{c \mathrm{EM}}^{1}(G ; \mathcal{A})
$$

(defined using continuous Eilenberg-MacLane cochains) is the quotient of the module of all continuous crossed homomorphisms $G \rightarrow \mathcal{A}$ by the submodule consisting of the coboundaries $h \mapsto h \cdot \alpha-\alpha, \alpha \in \mathcal{A}$. Therefore, $\widehat{F}_{s}$ defines an element

$$
\left[\widehat{F}_{s}\right] \in H_{c \mathrm{EM}}^{1}(\operatorname{Symp} ; \mathcal{A}) .
$$

Although there is no canonical choice for $\widehat{F}_{s}$, it turns out that the cohomology class $[\widehat{F}]:=\left[\widehat{F}_{s}\right]$ is independent of the choice of $s$.

We now define

$$
\operatorname{Ham}^{s}(M, \omega):=\operatorname{ker} \widehat{F}_{s} .
$$

\footnotetext{
${ }^{3}$ The group cohomology of a discrete group $G^{\delta}$ is defined algebraically using a cochain complex that in older literature is called the Eilenberg-MacLane complex. This cohomology is isomorphic to the singular cohomology of the classifying space $B G^{\delta}$. If $G$ is a topological group, then one can also consider the cohomology groups defined by restricting to continuous cochains. Since the resulting cohomology groups are completely different from the singular cohomology of $B G$, we will for the sake of clarity denote the group cohomology by $H_{\mathrm{EM}}^{*}$, adding a $c$ wherever appropriate to emphasize continuity.
} 
These groups depend on the representative $\widehat{F}_{s}$ chosen for the class $\left[\widehat{F}_{s}\right]$, but they are all conjugate via elements of $\operatorname{Symp}_{0}$. Moreover their intersection with the subgroup $\operatorname{Symp}_{H}$ of Symp that acts trivially on $H^{1}(M ; \mathbb{R})$ is independent of $s$ (see Lemma 2.5.). This holds because any crossed homomorphism $\widehat{F}$ : Symp $\rightarrow \mathcal{A}$ restricts to a homomorphism on Symp $_{H}$ that depends only on the class represented by $\widehat{F}$ in $H_{c \mathrm{EM}}^{1}(\operatorname{Symp} ; \mathcal{A})$.

Because $\mathrm{Ham}^{s}$ is the kernel of a continuous crossed homomorphism, it follows from standard theory that one can use this homomorphism to define a class

$$
\mathcal{O}^{M} \in H^{2}\left(B \text { Symp; } H_{\mathbb{Q}}\right)
$$

that measures the obstruction to lifting a map $\phi: B \rightarrow B$ Symp to $B \mathrm{Ham}^{s}$. Here one should think of $H_{\mathbb{Q}}:=H^{1}\left(M ; \mathcal{P}_{\omega}\right)$ as $\pi_{1}(\mathcal{A})$, where $\mathcal{A}:=H^{1}(M ; \mathbb{R}) / H^{1}\left(M ; \mathcal{P}_{\omega}\right)$.

The next result explains the role of this class $\mathcal{O}^{M}$. The statement in (i) below arose from some remarks in Gal-Kędra [3].

Theorem 1.10. (i) The obstruction class $\mathcal{O}^{M} \in H^{2}\left(B\right.$ Symp; $\left.H_{\mathbb{Q}}\right)$ equals the image $d_{2}^{\omega}([\omega])$ of $[\omega] \in H^{2}\left(M ; \mathcal{P}_{\omega}\right)$ under the differential $d_{2}^{\omega}$ in the LeraySerre spectral sequence for the cohomology of the universal $M$-bundle over $B$ Symp with coefficients $\mathcal{P}_{\omega}$.

(ii) There is a crossed homomorphism $\widetilde{F}:$ Symp $\rightarrow H^{1}(M ; \mathbb{R}) / \Gamma$ that extends Flux if and only if $\mathcal{O}^{M}$ lies in the image of $H^{2}(B$ Symp; $\Gamma)$ in $H^{2}\left(B\right.$ Symp; $\left.H_{\mathbb{Q}}\right)$.

Corollary 1.11. Ham ${ }^{s}$ has the extension property.

Proof. By Lemma 1.6 there is a closed extension of $\omega$ iff $d_{2}([\omega])=0$, where $d_{2}$ denotes the differential in the spectral sequence for real cohomology. But because $\mathcal{P}_{\omega}$ is divisible, this vanishes iff $d_{2}^{\omega}([\omega])=0$.

Corollary 1.12. The following conditions are equivalent:

(i) Every symplectic $M$-bundle has a closed connection form.

(ii) $\Gamma=0$ and there is a crossed homomorphism $\widetilde{F}: \operatorname{Symp} \rightarrow H^{1}(M ; \mathbb{R})$ extending Flux.

For example, when $(M, \omega)$ is monotone, the first Chern class of the vertical tangent bundle of $P \rightarrow B$ provides an extension of $[\omega]$. Therefore, the obstruction class $\mathcal{O}^{M}$ must vanish. This is consistent with the corollary since the Kotschick-Morita homomorphism $F_{\mathrm{KM}}$ extends Flux.

The next result clarifies the conditions under which Flux can be extended. In Kotschick-Morita [9, Section 6.3], the obstruction to the existence of an extension of Flux is described as a certain class $\delta([F]) \in H_{\mathrm{EM}}^{2}\left(\pi_{0}(\mathrm{Symp})\right.$; $\left.H^{1}(M ; \mathbb{R}) / \Gamma\right)$. Thus, the next proposition can be interpreted as giving geometric explanations of what it means for this class to vanish. 
Proposition 1.13. The following conditions are equivalent:

(i) Flux extends to a crossed homomorphism $\widetilde{F}: \operatorname{Symp}(M, \omega) \rightarrow H^{1}(M ; \mathbb{R}) / \Gamma$.

(ii) For every closed Riemann surface $\Sigma$, every representation of $\pi_{1}(\Sigma)$ in $\pi_{0}$ (Symp) lifts to a representation into the group Symp / Ham.

(iii) For every product of commutators $\left[u_{1}, u_{2}\right] \cdots\left[u_{2 p-1}, u_{2 p}\right], u_{i} \in$ Symp, that lies in $\mathrm{Symp}_{0}$, there are elements $g_{1}, \ldots, g_{2 p} \in \mathrm{Symp}_{0}$ such that

$$
\left[u_{1} g_{1}, u_{2} g_{2}\right] \cdots\left[u_{2 p-1} g_{2 p-1}, u_{2 p} g_{2 p}\right] \in \text { Ham . }
$$

(iv) For every symplectic $M$-bundle $P \rightarrow \Sigma$ there is a bundle $Q \rightarrow S^{2}$ such that the fiberwise connect sum $P \# Q \rightarrow \Sigma \# S^{2}=\Sigma$ has a closed connection form.

Remark 1.14. If we restrict to the subgroup $\operatorname{Symp}_{H}$ of Symp that acts trivially on $H^{1}(M ; \mathbb{R})$, then (iii) is equivalent to saying that

$$
\left[\operatorname{Symp}_{H}, \operatorname{Symp}_{H}\right] \cap \operatorname{Symp}_{0}=\operatorname{Ham} .
$$

We show in Corollary 4.11 that equation (1.3) holds when $[\omega]$ vanishes on tori and lies in the subring of $H^{*}(M)$ generated by $H^{1}$. However, it is so far unknown whether it always holds. If not, then $\widetilde{F}$ cannot always exist. On the other hand, there are indications that (1.3) might always hold. It seems that a large part of $\pi_{0}$ (Symp) can be generated by Dehn twists about Lagrangian spheres: cf. Seidel $[\mathbf{2 0}, 1.7]$. In dimensions $>2$, these are well defined up to Hamiltonian isotopy and act trivially on $H^{1}(M)$, and so one might be able to take $\mathcal{H} \cap \operatorname{Symp}_{H}$ to be the group generated by Dehn twists. In any case, it does not seem that the methods used in this paper are sufficiently deep to resolve this question.

1.4. Further results and remarks. After discussing stability, we describe a few cases where it is possible to extend the Flux homomorphism. We end by discussing the integral case and the question of uniqueness.

Stability under perturbations of $\omega$. It was shown in LalondeMcDuff [11] that Hamiltonian bundles are stable under small perturbations of $\omega$. One cannot expect general symplectic bundles to be stable under arbitrary small perturbations of $\omega$ since $\pi_{1}(B)$ may act nontrivially on $H^{2}(M ; \mathbb{R})$. Given a symplectic bundle $(M, \omega) \rightarrow P \rightarrow B$, let us denote by $V_{2}(P)$ the subspace of $H_{2}(M ; \mathbb{Q})$ generated by the elements $g_{*}(C)-C$, where $C \in H_{2}(M ; \mathbb{Z})$ and $g$ is any symplectomorphism of $M$ that occurs as the holonomy of a symplectic connection on $P \rightarrow B$ around some loop in $B$. (Since $g_{*}(C)$ depends only on the smooth isotopy class of $g$, it does not matter which connection we use.) The subspace $H^{2}(M ; \mathbb{R})^{\operatorname{inv}(P)}$, consisting of classes $a \in H^{2}(M ; \mathbb{R})$ that are fixed by all such $g$, is the annihilator of $V_{2}(P)$. The most one can expect is that the existence of a symplectic structure on $P \rightarrow B$ is stable under perturbations of $[\omega]$ in this subspace. For example, if 
$\omega$ is generic in the sense that it gives an injective map $H_{2}(M ; \mathbb{Z}) /$ Tor $\rightarrow \mathbb{R}$, then $V_{2}(P)$ is torsion and $H^{2}(M ; \mathbb{R})^{\operatorname{inv}(P)}=H^{2}(M ; \mathbb{R})$.

Proposition 1.15. Let $(M, \omega) \rightarrow P \rightarrow B$ be a symplectic $M$-bundle over a finite simplicial complex $B$. Then there is a neighborhood $\mathcal{N}(\omega)$ of $\omega$ in the space of all closed 2 -forms on $M$ that represent a class in $H^{2}(M ; \mathbb{R})^{\operatorname{inv}(P)}$ such that for all $\omega^{\prime} \in \mathcal{N}(\omega)$ :

(i) $P \rightarrow B$ has the structure of an $\omega^{\prime}$-symplectic bundle, and

(ii) if there is a closed extension of $\omega$, then the same is true for $\omega^{\prime}$.

Part (i) of this proposition follows by the arguments in [11, Corollary 2.5]. Part (ii) was also proved in $[\mathbf{1 1}]$ in the case when $B$ is classified by a map into $B \operatorname{Symp}_{0}(M, \omega)$. The proof of the general case is given at the end of Section 3. The next corollary is an immediate consequence of (ii).

Corollary 1.16. If $P \rightarrow B$ has a $\mathrm{Ham}^{s}$-structure, then the image of the restriction map $H^{2}(P ; \mathbb{R}) \rightarrow H^{2}(M ; \mathbb{R})$ is the subspace $H^{2}(M ; \mathbb{R})^{\operatorname{inv}(P)}$ of $H^{2}(M ; \mathbb{R})$ that is invariant under the action of $\pi_{1}(B)$.

This result implies that the differentials $d_{2}^{2,0}$ and $d_{3}^{2,0}$ in the Leray-Serre spectral sequence for the real cohomology of $P \rightarrow B$ vanish and so is a partial generalization of the vanishing results in $[\mathbf{1 1}]$.

Remark 1.17. Proposition 1.15 is proved using the Moser homotopy argument and so works only over compact pieces of Symp. This is enough to give stability for bundles over bases $B$ of finite homotopy type but is not enough to allow one to make any statements about properties that involve the full group Symp. Hence, even if Flux extends to $\widetilde{F}: \operatorname{Symp} \rightarrow H^{1}(M ; \mathbb{R}) / \Gamma$ for the manifold $(M, \omega)$, it is not clear that it also extends for sufficiently close forms $\omega^{\prime}$ whose cohomology class is invariant under $\operatorname{Symp}(M, \omega)$. For one thing, however close $\omega^{\prime}$ is, there may be new components of $\operatorname{Symp}\left(M, \omega^{\prime}\right)$ containing elements that are far from those in $\operatorname{Symp}(M, \omega)$.

Manifolds with $\Gamma=0$. One expects that for most manifolds, $\Gamma=0$. Whether $\mathcal{O}^{M}$ then vanishes is still not clear. We now discuss some special cases in which $\Gamma=0$ and Flux extends to a crossed homomorphism defined either on the whole group Symp or on some large subgroup.

The first case is when $(M, \omega)$ is (strongly) monotone, i.e., the symplectic class $[\omega]$ is a multiple of the first Chern class. In this case $\mathcal{O}^{M}=0$ since one can always choose a closed connection form in the class of a multiple of the vertical first Chern class. Kotschick-Morita [9] observed that Flux always extends. We shall give an explicit formula for $\widetilde{F}$ in Theorem 4.6. As noted in Remark 4.7 (ii), the argument in fact applies whenever [ $\omega]$ is integral and Symp has the integral extension property, i.e., there is a complex line bundle over the universal $M$-bundle $M_{\text {Symp }} \rightarrow B$ Symp whose first Chern class restricts to $[\omega]$ on the fiber. 
Another somewhat tractable case is when $(M, \omega)$ is atoroidal, i.e., $\int_{\mathbb{T}^{2}} \psi^{*} \omega=0$ for all smooth maps $\psi: \mathbb{T}^{2} \rightarrow M$. Note that $\Gamma=0$ for such manifolds because for each loop $\left\{f_{t}\right\}$ in $\operatorname{Symp}_{0}$, the value of the class $\operatorname{Flux}\left(\left\{f_{t}\right\}\right)$ on the 1-cycle $\gamma$ is obtained by integrating $\omega$ over the torus $\cup_{t} f_{t}(\gamma)$. In the next proposition, we denote by $\operatorname{Symp}_{\pi}$ the subgroup of Symp consisting of elements that are isotopic to a symplectomorphism that fixes the basepoint $x_{0}$ of $M$ and induces the identity map $\pi_{1}\left(M, x_{0}\right) \rightarrow \pi_{1}\left(M, x_{0}\right){ }^{4}$

Proposition 1.18. If $(M, \omega)$ is atoroidal, then $\Gamma=0$ and Flux extends to a homomorphism $\widetilde{F}: \operatorname{Symp}_{\pi} \rightarrow H^{1}(M ; \mathbb{R})$.

We shall see in Section 4.3 that in the above situation $\widetilde{F}$ can be extended to a crossed homomorphism defined on the whole of Symp but at the cost of enlarging the target group.

Proposition 1.18 gives a partial answer to Kȩdra-Kotschick-Morita's question [6] of whether the usual flux homomorphism $\operatorname{Symp}_{0} \rightarrow H^{1}(M ; \mathbb{R})$ extends to the full group Symp when $[\omega]$ is a bounded class. This condition means that $[\omega]$ may be represented by a singular cocycle that is uniformly bounded on the set of all singular 2 -simplices. ${ }^{5}$ If $[\omega]$ is bounded, then $(M, \omega)$ is atoroidal since an arbitrary multiple of a toric class $C$ can be represented by the sum of just two singular 2-simplices. Another interesting atoroidal case is that of symplectically hyperbolic manifolds. There are various possible definitions here. We shall use Polterovich's definition from [18] in which $(M, \omega)$ is called symplectically hyperbolic if the pullback $\widetilde{\omega}$ of $\omega$ to the universal cover $\widetilde{M}$ of $M$ has bounded primitive, i.e., $\widetilde{\omega}=d \beta$ for some 1-form $\beta$ that is bounded with respect to any metric on $\widetilde{M}$ that is pulled back from $M$. For example $(M, \omega)$ might be a product of Riemann surfaces of genus $>1$ with a product symplectic form. Because in the covering $\mathbb{R}^{2} \rightarrow \mathbb{T}^{2}$, the boundary of a square of side $N$ encloses $N^{2}$ fundamental domains, it is easy to check that any 2 -form on $\mathbb{T}^{2}$ whose pullback to $\mathbb{R}^{2}$ has bounded primitive must have zero integral over $\mathbb{T}^{2}$. Hence we find:

Lemma 1.19. Proposition 1.18 applies both when $[\omega]$ is bounded and when $(M, \omega)$ is symplectically hyperbolic.

Section 4.3 contains a few other similar results that are valid in special cases, for example, when $\omega$ vanishes on $\pi_{2}(M)$.

We end the introduction with some general remarks.

Remark 1.20. (The integral case). There is an analogous group $\operatorname{Ham}^{s \mathbb{Z}}$ which is the kernel of a crossed homomorphism $\widehat{F}_{s}^{\mathbb{Z}}$ with values

\footnotetext{
${ }^{4}$ One can check that $g \in \operatorname{Symp}_{\pi}$ iff for any path $\gamma$ in $M$ from $x_{0}$ to $g\left(x_{0}\right)$, the induced maps $\gamma_{*}, g_{*}: \pi_{1}\left(M, x_{0}\right) \rightarrow \pi_{1}\left(M, g x_{0}\right)$ differ by an inner automorphism. Thus, loosely speaking, $\operatorname{Symp}_{\pi}$ consists of all symplectomorphisms that act trivially on $\pi_{1}(M)$.

${ }^{5}$ The (smooth) cocycle represented by integrating $\omega$ can never be bounded because bounded cocycles vanish on cylinders as well as tori.
} 
in $H^{1}\left(M ; \mathbb{R} / \mathcal{P}_{\omega}^{\mathbb{Z}}\right)$ where $\mathcal{P}_{\omega}^{\mathbb{Z}}$ denotes the set of values of $[\omega]$ on the integral 2-cycles $H_{2}(M ; \mathbb{Z})$. In many respects, the properties of this group are similar to those of $\mathrm{Ham}^{s}$. However, there are some interesting differences. If Tor denotes the torsion subgroup of $H_{1}(M ; \mathbb{Z})$, then the analog of the group $\mathcal{A}$ occurring in equation (1.2) is

$$
\mathcal{A}^{\mathbb{Z}}:=H^{1}\left(M ; \mathbb{R} / \mathcal{P}_{\omega}^{\mathbb{Z}}\right) \cong \operatorname{Hom}\left(\operatorname{Tor}, \mathbb{R} / \mathcal{P}_{\omega}^{\mathbb{Z}}\right) \oplus H^{1}(M ; \mathbb{R}) / H^{1}\left(M ; \mathcal{P}_{\omega}^{\mathbb{Z}}\right) .
$$

Hence Theorem 1.10 (i) does not immediately generalize; the proof of Lemma 3.4 shows that the obstruction to the existence of a Ham ${ }^{s \mathbb{Z}}$-structure is two-fold, the first coming from the finite group $\pi_{0}\left(\mathcal{A}^{\mathbb{Z}}\right)$ (see Lemma 4.1) and the second an obstruction cocycle similar to $\mathcal{O}^{M}$ coming from $\pi_{1}\left(\mathcal{A}^{\mathbb{Z}}\right)$ (see Lemma 3.5). Nevertheless, since every $\mathrm{Ham}^{\mathrm{s}}$-bundle over a compact base $B$ has a finite cover with a $\operatorname{Ham}^{s \mathbb{Z}}$-structure, the latter group has the modified extension property.

The group $\operatorname{Ham}^{s \mathbb{Z}}$ is most interesting in the case when $\mathcal{P}_{\omega}^{\mathbb{Z}}=\mathbb{Z}$, i.e., when $[\omega]$ is a primitive integral class. In this situation, one might expect $B \mathrm{Ham}^{s \mathbb{Z}}$ to classify bundles $P \rightarrow M$ that have a closed and integral connection form. Even in the case when $\pi_{0}(\mathrm{Symp})$ acts trivially on $H_{1}(M ; \mathbb{Z})$; this is not quite true. Gal-Kędra [3] point out that there is a further torsion obstruction in $H^{3}(B ; \mathbb{Z})$ which measures whether the symplectic class $[\omega]$ has an integral rather than rational extension: cf. Proposition 3.3 below and Example 4.2.

If $\pi_{0}$ (Symp) acts nontrivially on Tor $\subset H_{1}(M ; \mathbb{Z})$, then the groups $\operatorname{Ham}^{s \mathbb{Z}}$ are not all conjugate. We show in Lemma 4.4 that up to homotopy the choice of splitting $s$ is equivalent to the choice of an integral lift $\tau$ of $[\omega]$, i.e., of a prequantum line bundle $L_{\tau}$. Moreover, $s$ itself is determined by a unitary connection $\alpha$ on $L$, and the group $\mathrm{Ham}^{s \mathbb{Z}}$ consists of all symplectomorphisms $\phi$ that preserve the monodromy of $\alpha$, i.e., for all closed loops $\gamma$ in $M$, the $\alpha$-monodromy $m_{\alpha}(\gamma)$ round $\gamma$ equals that round $\phi(\gamma)$. Thus, $\operatorname{Ham}^{s \mathbb{Z}}$ is the same as the group $D_{\ell}$ considered by Kostant in [8]. (It is also homotopy equivalent to the covering group of Symp considered in Gal-Kędra [3]; see Proposition 4.5 below.) Hence one can think of the monodromy $m_{\alpha}$ of $\alpha$ as a "Hamiltonian structure" on $M$, i.e., this function on the space $\mathcal{L} M$ of closed loops in $M$ is the structure on $M$ that is preserved by the elements of $\operatorname{Ham}^{s \mathbb{Z}}{ }^{6}$ Thus an $M$-bundle $P \rightarrow B$ with structure group $\mathrm{Ham}^{s \mathbb{Z}}$ has such monodromy functions on each fiber, but (just as in the case of the fiberwise

\footnotetext{
${ }^{6}$ The function $m_{\alpha}: \mathcal{L} M \rightarrow \mathbb{R} / \mathbb{Z}$ is characterized by the following two properties: (i) $m_{\alpha}(\beta * \gamma)=m_{\alpha}(\beta)+m_{\alpha}(\gamma)$, where $\beta * \gamma$ is the concatenation of two loops with the same base point; and (ii) if $\gamma$ is the boundary of a 2 -chain $W$, then $m_{\alpha}(\gamma)=\omega(W) \bmod \mathbb{Z}$. Hence it contains the same information as the splitting $s^{\mathbb{Z}}$. For general $[\omega]$, one can think of a Hamiltonian structure as the marking defined by the splitting $s$; cf. the discussion in the appendix of $[\mathbf{1 1}]$.
} 
symplectic form) these do not need to be induced by a global monodromy function coming from a line bundle over $P$. Such global structures are called integral configurations in Gal-Kȩdra [3], where the problem of classifying them is discussed.

Remark 1.21. (Issues of uniqueness) (i) Because we are interested in the algebraic and geometric properties of the symplectomorphism group, we restricted ourselves above to the case when $\mathcal{H}$ is a subgroup of Symp. However, from a homotopy theoretic point of view, it would be more natural to look for a group $\mathcal{K}$ that classifies pairs consisting of a symplectic $M$-bundle $\pi: P \rightarrow B$ together with an extension $\widetilde{a} \in H^{2}(P ; \mathbb{R})$ of the fiberwise symplectic class $[\omega]$. Here we should either normalize $\widetilde{a}$ by requiring $\pi_{!}\left(\widetilde{a}^{n+1}\right)=0$ (where $\pi$ ! denotes integration over the fiber) or consider $\widetilde{a}$ to be well defined modulo elements in $\pi^{*} H^{2}(B)$. Then the homotopy class of $B \mathcal{K}$ would be well defined and there would be a forgetful map $\psi: B \mathcal{K} \rightarrow B$ Symp which is well defined up to homotopy (assuming that we are working in the category of spaces with the homotopy type of a CW complex). In general, $\psi$ would not be a homotopy equivalence since the extension class $\widetilde{a} \in H^{2}(P ; \mathbb{R})$ could vary by an element in $H^{1}\left(B ; H^{1}(M ; \mathbb{R})\right)$. Further, in this scenario, $\mathcal{K}$ need not be a subgroup of Symp. (Cf. the discussion in Lalonde-McDuff [11] of the classification of Hamiltonian structures.)

(ii) If we insist that $\mathcal{K}$ be a subgroup of Symp, then there are several possible notions of equivalence, the most natural of which is perhaps given by conjugation by an element in Symp $_{0}$. With this definition equivalent groups would be isomorphic. We show in Section 2 that the groups $\mathrm{Ham}^{s}$ are equivalent in this sense, though when $H_{1}(M ; \mathbb{Z})$ has torsion, the integer versions $\operatorname{Ham}^{s \mathbb{Z}}$ may not be. It is also not clear whether any two groups $\mathcal{H}_{1}, \mathcal{H}_{2}$ that intersect each component of Symp and satisfy $\mathcal{H}_{1} \cap$ Symp $_{0}=\mathcal{H}_{2} \cap$ Symp $_{0}=$ Ham must be isomorphic as abstract groups, although any such group must be isomorphic to an extension of $\pi_{0}$ (Symp) by Ham. Moreover, there is no immediate reason why they should be conjugate. For example, suppose that the group $\pi_{0}$ (Symp) is isomorphic to $\mathbb{Z}$, generated by the component $\operatorname{Symp}_{\alpha}$ of Symp. Then because Ham is a normal subgroup of Symp, the subgroup $\mathcal{H}_{g}$ of Symp, generated by Ham together with any element $g \in \operatorname{Symp}_{\alpha}$, intersects $\mathrm{Symp}_{0}$ in Ham and therefore has the required properties. Any two such groups $\mathcal{H}_{g_{i}}, i=1,2$, are isomorphic, though they are conjugate only if there is $h \in$ Symp such that $g_{1} h g_{2}^{-1} h^{-1} \in$ Ham. On the other hand, because $g_{1}$ and $g_{2}$ can be joined by an isotopy, there is a smooth family of injective group homomorphisms $\iota_{t}: \mathcal{H}_{g_{1}} \rightarrow$ Symp, $t \in[1,2]$, that starts with the inclusion and ends with an isomorphism onto $\mathcal{H}_{g_{2}}$. Thus the homotopy properties of the inclusions $\mathcal{H}_{g_{i}} \rightarrow$ Symp are the same.

(iii) Instead of looking for subgroups of Symp with the extension property one could look for covering groups $\mathcal{H} \rightarrow$ Symp with this property. Notice 
that if $\Lambda$ is a discrete subgroup of an Abelian topological group $\mathcal{A}$ and if the continuous crossed homomorphism $F: G \rightarrow \mathcal{A} / \Lambda$ extends the composite $F_{0}: G_{0} \rightarrow \mathcal{A} \rightarrow \mathcal{A} / \Lambda$, where $f: G_{0} \rightarrow \mathcal{A}$ is a homomorphism defined on the identity component of $G_{0}$, then the fiber product

$$
\widetilde{G}:=\{(g, a) \in G \times \mathcal{A} \mid F(g)=a+\Lambda\}
$$

of $G$ and $\mathcal{A}$ over $\mathcal{A} / \Lambda$ is a covering group of $G$ that contains a copy of $G_{0}$, namely the graph of $f$. Moreover, the obvious projection $\widetilde{G} \rightarrow \mathcal{A}$ lifts $F_{0}$. This approach is particularly relevant in the integral case mentioned in Remark 1.20 above, as well as the cohomologically symplectic case, where the analog of the Hamiltonian group is already a covering group of Diff $_{0}$. For further discussion, see Section 4.4 and Gal-Kȩdra [3].

\section{Definition and Properties of $\widehat{F}_{s}$}

Define $\mathcal{P}_{\omega}^{\mathbb{Z}}$ (resp. $\left.\mathcal{P}_{\omega}:=\mathcal{P}_{\omega}^{\mathbb{Q}}\right)$ to be the set of values taken by $[\omega]$ on the elements of $H_{2}(M ; \mathbb{Z})$ (resp. $H_{2}(M ; \mathbb{Q})$ ). To define $\widehat{F}_{s}$ we follow a suggestion of Polterovich (explained in Lalonde-McDuff [11]). Define the homology group

$$
S H_{1}(M, \omega ; \mathbb{Z})
$$

to be the quotient of the space of integral 1-cycles in $M$ by the image under the boundary map $\partial$ of the integral 2-chains with zero symplectic area. Then there is a projection $\pi_{\mathbb{Z}}: S H_{1}(M, \omega ; \mathbb{Z}) \rightarrow H_{1}(M ; \mathbb{Z})$ and we set

$$
S H_{1}(M, \omega):=S H_{1}(M, \omega ; \mathbb{Z}) \otimes \mathbb{Q} .
$$

We shall consider $S H_{1}(M, \omega)$ and $\mathcal{P}_{\omega}^{\mathbb{Q}}$ as $\mathbb{Q}$-vector spaces. Given a loop (or integral 1 -cycle) $\ell$ in $M$, we denote its image in $H_{1}(M ; \mathbb{Z})$ or $H_{1}(M ; \mathbb{Q})$ by $[\ell]$ and its image in $S H_{1}(M, \omega ; \mathbb{Z})$ or $S H_{1}(M, \omega)$ by $\langle\ell\rangle$. We usually work over the rationals and shall omit the label $\mathbb{Q}$ unless there is a possibility of confusion.

Lemma 2.1. There are split exact sequences

$$
0 \longrightarrow \mathbb{R} / \mathcal{P}_{\omega}^{\mathbb{Z}} \longrightarrow S H_{1}(M, \omega ; \mathbb{Z}) \stackrel{\pi_{\mathbb{Z}}}{\longrightarrow} H_{1}(M ; \mathbb{Z}) \longrightarrow 0,
$$

and

$$
0 \longrightarrow \mathbb{R} / \mathcal{P}_{\omega}^{\mathbb{Q}} \longrightarrow S H_{1}(M, \omega) \stackrel{\pi}{\longrightarrow} H_{1}(M ; \mathbb{Q}) \longrightarrow 0 .
$$

Proof. Choose a continuous family of integral 2-chains $f_{t}: D \rightarrow M$ for $t \in \mathbb{R}$ with $\int_{D} f_{t}^{*} \omega=t$. If $\gamma_{t}:=\left.f_{t}\right|_{\partial D}$ denotes the boundary of $f_{t}$, then the elements

$$
\left\langle\gamma_{t}\right\rangle, \quad t \in \mathbb{R}
$$


generate the kernel of the projection $\pi_{\mathbb{Z}}: S H_{1}(M, \omega ; \mathbb{Z}) \rightarrow H_{1}(M, \mathbb{Z})$. Moreover, they represent different classes in $S H_{1}(M, \omega ; \mathbb{Z})$ if and only if $t-t^{\prime} \notin \mathcal{P} \underset{\omega}{\mathbb{Z}}$. Hence, the sequence

$$
0 \longrightarrow \mathbb{R} / \mathcal{P}_{\omega}^{\mathbb{Z}} \longrightarrow S H_{1}(M, \omega ; \mathbb{Z}) \stackrel{\pi_{\mathbb{Z}}}{\longrightarrow} H_{1}(M ; \mathbb{Z}) \rightarrow 0
$$

is exact. To see that it splits, we just need to check that each element $\lambda=[\ell]$ of finite order $N$ in $H_{1}(M ; \mathbb{Z})$ is the image of some element of order $N$ in $S H_{1}(M ; \mathbb{Z})$. But if $W$ is an integral 2-chain such that $\partial W=N \ell$ and if $\mu:=\int_{W} \omega$, then

$$
N\left(\langle\ell\rangle-\left\langle\gamma_{\mu / N}\right\rangle\right)=0 \quad \text { and } \quad \pi_{\mathbb{Z}}\left(\langle\ell\rangle-\left\langle\gamma_{\mu / N}\right\rangle\right)=[\ell]
$$

In fact every element of order $N$ in the coset $\pi_{\mathbb{Z}}^{-1}([\ell])$ has the form $\langle\ell\rangle-\left\langle\gamma_{\nu}\right\rangle$, where $N \nu \in \mu+\mathcal{P}_{\omega}^{\mathbb{Z}}$. The proof for (2.2) is similar.

We explain in Section 4.1 a natural way to understand splittings of $\pi_{\mathbb{Z}}$ in the case when $\mathcal{P}_{\omega}^{\mathbb{Z}}=\mathbb{Z}$ : cf. Definition 4.3. Note also that in the previous lemma, there is no need for $\omega$ to be nondegenerate; it suffices for it to be closed. However, if it were an arbitrary closed form, it would not have many isometries, and so the next lemmas would have little interest.

Lemma 2.2. The group $\operatorname{Symp}(M, \omega)$ acts on $S H_{1}(M, \omega ; \mathbb{Z})$ and $S H_{1}(M, \omega)$. The induced action of $\mathrm{Symp}_{0}$ on the set of splittings of $\pi$ is transitive. When $H_{1}(M ; \mathbb{Z})$ has no torsion Symp also acts transitively on the splittings of $\pi_{\mathbb{Z}}$.

Proof. Again, we shall work with the sequence over $\mathbb{Z}$. The group $\operatorname{Symp}(M, \omega)$ acts on these spaces because it preserves $\omega$. To prove the transitivity statement, note first that any splitting $s$ of $\pi_{\mathbb{Z}}$ has the form $s \lambda_{i}=\left\langle\ell_{i}\right\rangle$, where $\ell_{1}, \ldots, \ell_{k}$ are loops (i.e., integral 1-cycles) in $M$ that project to the basis $\lambda_{1}, \ldots, \lambda_{k}$ of $H_{1}(M ; \mathbb{Z})$. Suppose given two such splittings $s, s^{\prime}$ corresponding to different sets $L, L^{\prime}$ of representing 1-cycles for the $\lambda_{i}$. Suppose also that $\operatorname{dim} M>2$. Since Hamiltonian isotopies have zero flux, we may move the loops in $L$ and $L^{\prime}$ by such isotopies, without affecting their images in $S H_{1}(M, \omega ; \mathbb{Z})$ and so that no two intersect. Now choose $T_{1}, \ldots, T_{k} \in \mathbb{R}^{+}$ such that

$$
\left\langle\ell_{i}^{\prime}\right\rangle=\left\langle\ell_{i}\right\rangle+\left\langle\gamma_{T_{i}}\right\rangle, \quad 1 \leq i \leq k,
$$

where the $\gamma_{t}$ are as in Lemma 2.1. For each $i$, there is a symplectic isotopy $h_{i, t}$ such that for all $t \in\left[0, T_{i}\right]$,

$$
\left.h_{i, t}\right|_{\ell_{j}}=\mathrm{id}, \quad j<i,\left.\quad h_{i, t}\right|_{h_{j, T_{j}} \ell_{j}}=\mathrm{id}, j>i, \quad \int_{W_{i}} \omega=T_{i},
$$

where $W_{i}:=\cup_{0 \leq t \leq T_{i}} h_{i, t}\left(\ell_{i}\right)$. (Take the $h_{i, t}$ to be generated by closed 1-forms $\alpha_{i}$ that vanish near the appropriate loops and are such that $\int_{\ell_{i}} \alpha_{i} \neq 0$. Here, we are using the fact that $\left[\ell_{i}\right]$ is not a torsion class.) Then $h:=$ $h_{1, T_{1}} \circ \cdots \circ h_{k, T_{k}}$ takes $s$ to $s^{\prime}$. 
To extend this argument to the case $\operatorname{dim} M=2$, it is convenient to describe the splitting by its effect on a standard basis $\lambda_{i}$ of $H_{1}(M ; \mathbb{Z})$. Thus we may assume that $\ell_{i}$ and $\ell_{j}$ are disjoint unless $(i, j)=(2 k-1,2 k)$ in which case they intersect in a single point. If $s_{0}$ is the splitting defined by these loops, it suffices to show that for any numbers $T_{i}$, there are representatives $\ell_{i}^{\prime}$ for the $\left[\ell_{i}\right]$ such that for each $i$ there is a cylinder of area $T_{i}$ with boundary $\ell_{i}^{\prime}-\ell_{i}$. One achieves this by first isotoping the $\ell_{i}$ for $i$ odd (fixing the other loops) and then adjusting the $\ell_{i}$ for even $i$.

Choose a splitting $s$ for $\pi_{\mathbb{Z}}$. If $h \in \operatorname{Symp}$ and $\lambda \in H_{1}(M ; \mathbb{Z})$, then the element $h_{*}(s \lambda)-s\left(h_{*} \lambda\right)$ lies in the kernel of $\pi_{\mathbb{Z}}: S H_{1}(M, \omega) \rightarrow H_{1}(M ; \mathbb{Z})$ and one can define a map

$$
\widehat{F}_{s}^{\mathbb{Z}}: \operatorname{Symp}(M) \rightarrow \mathcal{A}^{\mathbb{Z}}:=\operatorname{Hom}\left(H_{1}(M ; \mathbb{Z}), \mathbb{R} / \mathcal{P}_{\omega}^{\mathbb{Z}}\right)
$$

by setting

$$
\widehat{F}_{s}^{\mathbb{Z}}(h)(\lambda):=h_{*}(s \lambda)-s\left(h_{*} \lambda\right) \in \mathbb{R} / \mathcal{P}_{\omega}^{\mathbb{Z}}, \quad \lambda \in H_{1}(M ; \mathbb{Z}) .
$$

Explicitly, if we denote by $\bar{\lambda}$ the image $s(\lambda)$ of $\lambda \in H_{1}(M)$, then

$$
\widehat{F}_{s}(h)(\lambda)=a(h \bar{\lambda}-\overline{h \lambda}),
$$

where $a\left(\left\langle\ell^{\prime}\right\rangle-\langle\ell\rangle\right)$ is the symplectic area of any cycle with boundary $\ell^{\prime}-\ell$. Similarly, for each splitting $s$ of (2.2), we define

$$
\widehat{F}_{s}: \operatorname{Symp}(M) \longrightarrow \mathcal{A}:=\operatorname{Hom}\left(H_{1}(M), \mathbb{R} / \mathcal{P}_{\omega}^{\mathbb{Q}}\right)=H^{1}(M ; \mathbb{R}) / H^{1}\left(M ; \mathcal{P}_{\omega}^{\mathbb{Q}}\right)
$$

by

$$
\widehat{F}_{s}(h)(\lambda):=h_{*}(s \lambda)-s\left(h_{*} \lambda\right) \in \mathbb{R} / \mathcal{P}_{\omega}^{\mathbb{Q}}, \quad \lambda \in H_{1}(M ; \mathbb{Q}) .
$$

Proposition 2.3. (i) $\widehat{F}_{s}^{\mathbb{Z}}$ is a crossed homomorphism that equals the composite

$$
\operatorname{Symp}_{0} \stackrel{\text { Flux }}{\longrightarrow} H^{1}(M ; \mathbb{R}) / \Gamma \longrightarrow \mathcal{A}^{\mathbb{Z}}:=\operatorname{Hom}\left(H_{1}(M ; \mathbb{Z}), \mathbb{R} / \mathcal{P}_{\omega}^{\mathbb{Z}}\right)
$$

on $\mathrm{Symp}_{0}$. Moreover, the class

$$
\left[\widehat{F}^{\mathbb{Z}}\right]:=\left[\widehat{F}_{s}^{\mathbb{Z}}\right] \in H_{c \mathrm{EM}}^{1}\left(\operatorname{Symp}, \mathcal{A}^{\mathbb{Z}}\right)
$$

is independent of the choice of $s$.

(ii) The analogous statements hold for $\widehat{F}_{s}$. 
Proof. $\widehat{F}_{s}^{\mathbb{Z}}$ is a crossed homomorphism because for all $g, h \in$ Symp

$$
\begin{aligned}
\widehat{F}_{s}^{\mathbb{Z}}(g h)(\lambda) & =a(g h \bar{\lambda}-\overline{g h \lambda}) \\
& =a(g h \bar{\lambda}-g \overline{h \lambda})+a(g \overline{h \lambda}-\overline{g h \lambda}) \\
& =a(h \bar{\lambda}-\overline{h \lambda})+a(g \overline{h \lambda}-\overline{g h \lambda}) \\
& =\widehat{F}_{s}^{\mathbb{Z}}(h)(\lambda)+\widehat{F}_{s}^{\mathbb{Z}}(g)(h \lambda) \\
& =\widehat{F}_{s}^{\mathbb{Z}}(h)(\lambda)+h^{*} \widehat{F}_{s}^{\mathbb{Z}}(g)(\lambda) .
\end{aligned}
$$

The rest of the first statement in (i) is immediate from the definition.

To prove the second statement in (i) observe that two choices of splitting $s, s^{\prime}$ differ by the element $\alpha \in \mathcal{A}^{\mathbb{Z}}:=\operatorname{Hom}\left(H_{1}(M ; \mathbb{Z}), \mathbb{R} / \mathcal{P}_{\omega}^{\mathbb{Z}}\right)$ given by

$$
\alpha(\lambda):=s^{\prime}(\lambda)-s(\lambda) \in \mathbb{R} / \mathcal{P}_{\omega}^{\mathbb{Z}}, \quad \lambda \in H_{1}(M ; \mathbb{Z}) .
$$

It follows easily that

$$
\widehat{F}_{s^{\prime}}^{\mathbb{Z}}(h)-\widehat{F}_{s}^{\mathbb{Z}}(h)=\alpha-h^{*} \alpha,
$$

and so is a coboundary.

The proof of (ii) is similar.

Definition 2.4. Given a splitting $s: H_{1}(M ; \mathbb{Q}) \rightarrow S H_{1}(M, \omega)$, we define the enlarged Hamiltonian group $\operatorname{Ham}^{s}(M, \omega)$ to be the kernel of $\widehat{F}_{s}$. Similarly, we define $\operatorname{Ham}^{s \mathbb{Z}}(M, \omega)$ to be the kernel of the integral crossed homomorphism $\widehat{F}_{s}^{\mathbb{Z}}$.

Lemma 2.5. Let $s, s^{\prime}$ be two splittings and define $\operatorname{Symp}_{H}$ to be the subgroup of Symp that acts trivially on rational homology.

(i) $\operatorname{Symp}_{H} \cap \operatorname{Ham}^{s}=\operatorname{Symp}_{H} \cap \operatorname{Ham}^{s^{\prime}}$.

(ii) The map $\pi_{0}\left(\mathrm{Ham}^{s}\right) \rightarrow \pi_{0}$ (Symp) is surjective.

(iii) The subgroups $\mathrm{Ham}^{s}$ and $\mathrm{Ham}^{s^{\prime}}$ are conjugate in Symp by an element in Symp $_{0}$.

(iv) When topologized as a subspace of Symp, the path component of $\mathrm{Ham}^{s}$ containing the identity element is $\operatorname{Ham}(M, \omega)$.

Proof. (i) is an immediate consequence of the identity (2.5). (iii) follows from the fact that $\mathrm{Symp}_{0}$ acts transitively on the set of splittings and the description of $\mathrm{Ham}^{s}$ as the subgroup of Symp whose action on $S H_{1}(M, \omega)$ preserves the image of $s$. To prove (ii), we must show that any element $h \in$ Symp is homotopic to an element in Hams ${ }^{s}$. This holds because the splittings $s^{\prime}=h_{*}(s)$ and $s$ are conjugate by an element in $\mathrm{Symp}_{0}$. To prove (iv), consider a continuous path $h_{t} \in$ Symp that starts at the identity and is such that $\widehat{F}_{s}\left(h_{t}\right)(\lambda)=0 \in \mathbb{R} / \mathcal{P}_{\omega}$ for all $t$. By Proposition 2.3, the path $t \mapsto \widehat{F}_{s}\left(h_{t}\right)(\lambda) \in \mathbb{R} / \mathcal{P}_{\omega}$ has the continuous lift $t \mapsto \operatorname{Flux}\left(h_{t}\right)(\lambda) \in \mathbb{R}$. Since 
$\mathcal{P}_{\omega}$ is totally disconnected this lift must be identically zero; in other words, the path $h_{t}$ is a Hamiltonian isotopy.

Part (iv) of Lemma 2.5 holds for the group $\operatorname{Ham}^{s \mathbb{Z}}$, and (i) holds if one replaces $\operatorname{Symp}_{H}$ by the group that acts trivially on $H_{1}(M ; \mathbb{Z})$. However, one must take care with the other two statements. For further details see Section 4.1 .

Remark 2.6. (Topologies on $\mathrm{Ham}^{s}$ and $\mathbb{R} / \mathcal{P}_{\omega}$.) The intersection $\mathrm{Ham}^{s} \cap \mathrm{Symp}_{0}$ is disconnected. In fact, it is everywhere dense in Symp $\mathbf{P}_{0}$. Hence, the subspace topology $\tau_{s}$ on $\mathrm{Ham}^{s}$ is rather counterintuitive and it is better to give $\mathrm{Ham}^{s}$ a finer topology in which its path components are closed. Therefore, although we give the group Symp the usual $C^{\infty}$-topology (which is the subspace topology it inherits from the diffeomorphism group), we give $\mathrm{Ham}^{s}$ the topology $\tau_{c}$ that it inherits from the Hamiltonian topology on Symp. Then the identity map $\left(\mathrm{Ham}^{s}, \tau_{c}\right) \rightarrow\left(\mathrm{Ham}^{s}, \tau_{s}\right)$ is continuous and is a weak homotopy equivalence. Thus this change in topology does not affect the homotopy or (co)homology of the space.

Correspondingly we shall always think of $\mathcal{P}_{\omega}$ as a discrete group. Further we think of quotients such as $\mathbb{R} / \mathcal{P}_{\omega}$ (or $\mathcal{A}$ ) as quasitopological spaces, ${ }^{7}$ i.e., we specify which maps $f: X \rightarrow \mathbb{R} / \mathcal{P}_{\omega}$ are continuous, where $X$ is a finite simplicial complex. This gives enough structure for $\mathbb{R} / \mathcal{P}_{\omega}$ to have well defined homotopy groups and hence a well defined weak homotopy type. In the present situation, we say that $f$ is continuous iff $X$ has a triangulation $X^{\prime}$ such that the restriction of $f$ to each simplex in $X^{\prime}$ has a continuous lift to $\mathbb{R}$. Hence

$$
\pi_{1}\left(\mathbb{R} / \mathcal{P}_{\omega}\right) \cong \mathcal{P}_{\omega}, \quad \pi_{j}\left(\mathbb{R} / \mathcal{P}_{\omega}\right)=0, \quad j>1
$$

Thus, $\mathbb{R} / \mathcal{P}_{\omega}$ is (weakly homotopic to) the Eilenberg-MacLane space $K\left(\mathcal{P}_{\omega}, 1\right)$. In some situations (such as Lemma 3.4 below), it is useful to replace the quasitopological space $\mathbb{R} / \mathcal{P}_{\omega}$ by its homotopy quotient $\mathbb{R} / / \mathcal{P}_{\omega}:=$ $\mathbb{R} \times \mathcal{P}_{\omega} E \mathcal{P}_{\omega}$. Here $E \mathcal{P}_{\omega}$ denotes a contractible space on which the group $\mathcal{P}_{\omega}$ acts freely and the notation $/ /$ is taken from Segal [19]. Since there is a homotopy fibration $\mathcal{P}_{\omega} \rightarrow E \mathcal{P}_{\omega} \rightarrow \mathbb{R} / / \mathcal{P}_{\omega}, \mathbb{R} / / \mathcal{P}_{\omega}$ is a $K\left(\mathcal{P}_{\omega}, 1\right)$. Hence, the map $\mathbb{R} / / \mathcal{P}_{\omega} \rightarrow \mathbb{R} / \mathcal{P}_{\omega}$ induced by collapsing $E \mathcal{P}_{\omega}$ to a point is a weak homotopy equivalence.

Remark 2.7. If $g \in$ Symp and $h \in \operatorname{Symp}_{0}$, then it is easy to check that Flux $\left(g^{-1} h g\right)=g^{*}($ Flux $h)$. Hence, if $\operatorname{Symp}_{H}$ denotes the subgroup of Symp acting trivially on $H^{1}(M ; \mathbb{R})$, then

$$
\left[\operatorname{Symp}_{0}, \operatorname{Symp}_{H}\right]=\text { Ham . }
$$

\footnotetext{
${ }^{7}$ This meaning of the word "quasitopological" seems to be obsolete, though it is very convenient. Its definition is analogous to that of a diffeology; cf. Iglesias et al. [5].
} 
On the other hand $\left[\operatorname{Symp}_{0}\right.$, Symp $]=$ Ham only if Symp $=\operatorname{Symp}_{H}$. Hence when Symp $\neq \operatorname{Symp}_{H}$, the flux homomorphism does not extend to a homomorphism Symp $\rightarrow H^{1}(M) / \Gamma$.

There is another relevant subgroup, namely $\operatorname{Symp}_{H^{\mathbb{Z}}}$, consisting of elements that act trivially on $H_{1}(M ; \mathbb{Z})$. Note that $\left[\operatorname{Symp}_{H^{\mathbb{Z}}}, \operatorname{Symp}_{H^{\mathbb{Z}}}\right] \cap \operatorname{Symp}_{0}$ lies in $\operatorname{Ham}^{s \mathbb{Z}}$ because $\widehat{F}_{s}^{\mathbb{Z}}$ restricts to a homomorphism on $\operatorname{Symp}_{H^{\mathbb{Z}}}$ and so

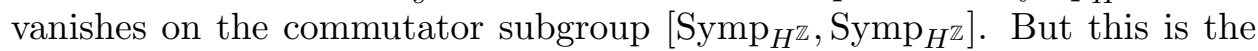
best we can say; in particular, it is not clear whether $\left[\operatorname{Symp}_{H}, \operatorname{Symp}_{H}\right] \cap$ Symp $_{0}$ must always equal Ham.

Lemma 2.8. The following statements are equivalent.

(i) $\left[\operatorname{Symp}_{H}, \operatorname{Symp}_{H}\right] \cap \mathrm{Symp}_{0}=\mathrm{Ham}$;

(ii) For every product of commutators $y:=\left[u_{1}, u_{2}\right] \cdots\left[u_{2 p-1}, u_{2 p}\right], u_{i} \in$ Symp $_{H}$, that lies in Symp $_{0}$, there are elements $g_{1}, \ldots, g_{2 p} \in$ Symp $_{0}$ such that

$$
f:=\left[u_{1} g_{1}, u_{2} g_{2}\right] \cdots\left[u_{2 p-1} g_{2 p-1}, u_{2 p} g_{2 p}\right] \in \text { Ham . }
$$

(iii) The flux homomorphism Flux : $\operatorname{Symp}_{0} \rightarrow H^{1}(M ; \mathbb{R}) / \Gamma$ extends to a continuous homomorphism $F: \operatorname{Symp}_{H} \rightarrow H^{1}(M ; \mathbb{R}) / \Gamma$.

Proof. Clearly (iii) implies (i), which in turn implies (ii). To see that (ii) implies (i), note the identity

$$
[u g, v h]=g^{u} h^{u v}\left(g^{-1}\right)^{u v}\left(h^{-1}\right)^{u v u^{-1}}[u, v],
$$

where $g^{a}:=a g a^{-1}$. It follows that $f y^{-1}$ may be written as a product of terms of the form $g_{2 i-1}^{\prime} g_{2 i}^{\prime}\left(g_{2 i-1}^{\prime \prime}\right)^{-1}\left(g_{2 i}^{\prime \prime}\right)^{-1}$, where $g_{j}^{\prime}$ and $g_{j}^{\prime \prime}$ are conjugate to $g_{j}$ by products of the $u_{i}$. Since the $u_{i}$ lie in $\operatorname{Symp}_{H}, \operatorname{Flux}\left(g_{j}^{\prime}\right)=\operatorname{Flux}\left(g_{j}^{\prime \prime}\right)=$ Flux $g_{j}$. Hence Flux $y=$ Flux $f=0$, and $y \in$ Ham.

It remains to show that (i) implies (iii). As in the proof of Proposition 1.8 given in Section 1, it suffices to find a section

$$
\sigma: \pi_{0}\left(\operatorname{Symp}_{H}\right) \longrightarrow \operatorname{Symp}_{H}, \quad \alpha \longmapsto \sigma_{\alpha} \in \operatorname{Symp}_{\alpha},
$$

such that

$$
\sigma_{\alpha \beta} \sigma_{\beta}^{-1} \sigma_{\alpha}^{-1} \in \operatorname{Ham}, \quad \alpha, \beta \in \pi_{0} \text { (Symp). }
$$

We first define $\sigma$ on the commutator subgroup $\left[\pi_{0}\left(\operatorname{Symp}_{H}\right), \pi_{0}\left(\operatorname{Symp}_{H}\right)\right]$. When $\alpha$ lies in this group, then the component $\operatorname{Symp}_{\alpha}$ contains elements that are products of commutators. We define $\sigma_{\alpha}$ to be such an element. Then $\sigma_{\alpha}$ is well defined modulo an element in Ham because $\left[\operatorname{Symp}_{H}, \operatorname{Symp}_{H}\right] \cap \mathrm{Symp}_{0}=$ Ham by assumption. Hence (2.6) holds for these $\alpha$. Now we extend by hand, defining a lift on the Abelian group $\pi_{0}\left(\operatorname{Symp}_{H}\right) /\left[\pi_{0}\left(\operatorname{Symp}_{H}\right), \pi_{0}\left(\operatorname{Symp}_{H}\right)\right]$. This is easy to do on the free part, and on the torsion part one uses the divisibility of $H^{1}(M ; \mathbb{R}) / \Gamma$. Note that $F$ is necessarily continuous since it is continuous on $\operatorname{Symp}_{0}$. 


\section{Bundles with structural group $\mathrm{Ham}^{\mathrm{s}}$}

This section contains the proofs of the main results about the group $\mathrm{Ham}^{s}$ and the obstruction class. In Section 3.1, we give a simple proof that Symp ${ }^{\text {Htop }}$ has the extension property (Proposition 1.1). Because $\mathrm{Ham}^{s}$ is geometrically defined, a similar argument shows that $\mathrm{Ham}^{s}$ has the extension property when restricted to bundles $P \rightarrow B$, where $\pi_{1}(B)$ acts trivially on $H_{1}(M ; \mathbb{Q})$. However, in the case when $[\omega]$ is integral, the analogous statement for $\mathrm{Ham}^{s \mathbb{Z}}$ holds only under additional hypotheses: see Proposition 3.3.

We start Section 3.2 by defining the obstruction cocycle $\mathcal{O}^{M}$ and proving Theorem 1.10. (As noted by Tsemo [21], this is a special case of a more general theory that can be nicely expressed in the language of gerbes.) We then prove Propositions 1.13, 1.3 and 1.4. The section ends with a proof of the stability result Proposition 1.15.

3.1. Groups with the extension property. We begin by proving Proposition 1.1 which states that the group Symp ${ }^{\text {Htop }}$ has the extension property.

Proof of Proposition 1.1. Suppose first that a smooth $M$-bundle $P \rightarrow B$ has a closed connection form $\Omega$. Because the holonomy of the corresponding connection is Hamiltonian round all contractible loops, it defines a continuous map from the space of based loops in $B$ to the group Symp ${ }^{\text {Htop }}$. This deloops to a lift $B \rightarrow B$ Symp $^{\text {Htop }}$ of the classifying map for $P \rightarrow B$. Therefore, the classifying map of any bundle with a closed connection form does lift to $B$ Symp ${ }^{\text {Htop }}$.

Conversely, consider the universal $M$-bundle

$$
M_{\text {Symp }}{ }^{\mathrm{Htop}} \rightarrow B \text { Symp }^{\mathrm{Htop}} \text {. }
$$

It suffices to show that the fiberwise symplectic class $a=[\omega]$ extends to a class $\widetilde{a} \in H^{2}\left(M_{\mathrm{Symp}}{ }_{\mathrm{Htop}} ; \mathbb{R}\right)$. If not, there is a map of a finite $\mathrm{CW}$ complex $X \rightarrow B$ Symp $^{\text {Htop }}$ such that the fiberwise symplectic class in the pullback bundle $M \rightarrow P_{X} \rightarrow X$ does not extend to $P_{X}$. By embedding $X$ in Euclidean space and replacing it by a small open neighborhood, we may assume that $X$ is a smooth (open) manifold. Hence, we may suppose that $M \rightarrow P_{X} \rightarrow X$ is smooth. Since the structural group is Symp ${ }^{\text {Htop }}$, this bundle has a symplectic connection with holonomy in Symp ${ }^{\text {Htop }}$. The holonomy round contractible loops lies in the identity component of Symp ${ }^{\text {Htop }}$ and hence is Hamiltonian. Therefore, the GLS construction provides a closed connection form $\tau$ on $P_{X}$ that defines this connection: see $[\mathbf{1 5}$, Theorem 6.21]. Since $[\tau] \in H^{2}\left(P_{X}\right)$ extends $[\omega]$, this contradicts our initial assumption.

Corollary 3.1. Let $\mathcal{H}$ be any subgroup of Symp whose identity component is contained in Ham. Consider the universal M-bundle

$$
M \longrightarrow M_{\mathcal{H}} \longrightarrow B \mathcal{H} \text {. }
$$


Then the fiberwise symplectic class $a:=[\omega]$ extends to $\widetilde{a} \in H^{2}\left(M_{\mathcal{H}} ; \mathbb{R}\right)$.

Proof. The hypothesis on $\mathcal{H}$ implies that the inclusion $\mathcal{H} \rightarrow$ Symp factors continuously through Symp ${ }^{\text {Htop }}$. Therefore, the class $\widetilde{a} \in H^{2}\left(M_{\mathrm{Symp}}{ }^{\mathrm{Htop}} ; \mathbb{R}\right)$ constructed above pulls back to $H^{2}\left(M_{\mathcal{H}}\right)$.

Lemma 3.2. If $\mathcal{P}_{\omega}^{\mathbb{Z}}=\mathbb{Z}$, then the universal $M$-bundle over $B \mathrm{Ham}^{\text {s }} \mathbb{Z}$ carries an extension of $[\omega]$ that takes integral values on all cycles lying over the 1-skeleton of the base.

Proof. The universal $M$-bundle over $B \mathrm{Ham}^{s \mathbb{Z}}$ carries a connection with holonomy in $\operatorname{Ham}^{s \mathbb{Z}}$. Since this has Hamiltonian holonomy round closed loops, the GLS construction shows that it is given by a closed connection form $\Omega$. We claim that $[\Omega]$ takes integral values on all cycles in $\pi^{-1}\left(B_{1}\right)$, where $B_{1}$ is the 1 -skeleton of $B \mathrm{Ham}^{s \mathbb{Z}}$.

Since $[\omega]$ is assumed integral, we need only check that $[\Omega]$ takes integral values on cycles $C(\gamma, \delta)$ formed as follows. Suppose that $\gamma$ is a closed path in the base with holonomy $m_{\gamma}: M \rightarrow M$ that fixes the class $\delta \in H_{1}(M ; \mathbb{Z})$. Choose a loop $\ell_{\delta}$ in $M$ such that $\left\langle\ell_{\delta}\right\rangle=s(\delta)$, and define $C(\gamma, \delta)$ to be the union of the cylinder $C^{\prime}$ formed by the parallel translation of $\ell_{\delta}$ around $\gamma$ with a chain $C^{\prime \prime}$ in $M$ with boundary $\ell_{\delta}-m_{\gamma}\left(\ell_{\delta}\right)$. Now observe that since $\Omega=0$ on $C^{\prime}$ and $m_{\gamma} \in \operatorname{Ham}^{s \mathbb{Z}}$, equation (2.4) implies that

$$
\int_{C(\gamma, \delta)} \Omega=\int_{C^{\prime \prime}} \omega=-\widehat{F}_{s}^{\mathbb{Z}}\left(m_{\gamma}\right) \in \mathcal{P}_{\omega}^{\mathbb{Z}} \subset \mathbb{Z}
$$

This completes the proof.

Proposition 3.3. (i) Let $P \rightarrow B$ be a symplectic bundle over a finite simplicial complex $B$ such that $\pi_{1}(B)$ acts trivially on $H_{1}(M ; \mathbb{R})$. Then $P$ has a closed connection form iff the classifying map for $P \rightarrow B$ lifts to $B \mathrm{Ham}^{s}$. (ii) Let $\pi: P \rightarrow B$ be a symplectic bundle over a finite simplicial complex $B$ such that $\pi_{1}(B)$ acts trivially on $H_{1}(M ; \mathbb{Z})$. Suppose further that either $H_{2}(B ; \mathbb{Z})$ is free or $P \rightarrow B$ admits a section over its 3 -skeleton. Then $P$ has a closed and integral connection form iff the classifying map for $P \rightarrow B$ lifts to $B \mathrm{Ham}^{s \mathbb{Z}}$.

Proof. Corollary 3.1 shows that every $\mathrm{Ham}^{\mathrm{s}}$-bundle has a closed connection form. Conversely, suppose that $P \rightarrow B$ has a closed connection form. Then the restriction map $H^{2}(P ; \mathbb{R}) \rightarrow H^{2}(M ; \mathbb{R})$ contains $[\omega]$ in its image. Because $\mathbb{Q}$ is a field, the restriction map $H^{2}\left(P ; \mathcal{P}_{\omega}^{\mathbb{Q}}\right) \rightarrow H^{2}(M ; \mathcal{P} \stackrel{\mathbb{Q}}{\omega})$ also contains $[\omega]$ in its image. Choose a class $a \in H^{2}\left(P ; \mathcal{P}_{\omega}^{\mathbb{Q}}\right)$ that extends $[\omega]$. Thurston's construction (cf. [15, Theorem 6.3]) provides a closed extension $\Omega$ in class $a$. We claim that the holonomy of $\Omega$ round loops $\gamma$ in the base $B$ lies in $\mathrm{Ham}^{s}$. Granted this, one can use the local trivializations given by $\Omega$ to reduce the structural group to $\mathrm{Ham}^{s}$. 
To prove the claim, observe that because $\pi_{1}(B)$ acts trivially on $H_{1}(M ; \mathbb{R})$ one can use the connection defined by $\Omega$ to construct for each loop $\gamma$ in $B$ a 2-cycle $C\left(\gamma, \delta_{i}\right)$ as in Lemma 3.2 , where $\left[\delta_{i}\right]$ runs through a basis of $H_{1}(M ; \mathbb{Q})$. Then, the $\Omega$-holonomy $m_{\Omega}(\gamma): M \rightarrow M$ round the loop $\gamma$ in $B$ satisfies the identity

$$
\widehat{F}_{s}\left(m_{\Omega}(\gamma)\right)\left(\delta_{i}\right)=-\int_{C\left(\gamma, \delta_{i}\right)} \Omega \in \mathcal{P}_{\omega}^{\mathbb{Q}} .
$$

Hence, $m_{\Omega}(\gamma) \in \operatorname{Ham}^{s}$. This completes the proof of (i).

Now consider (ii). If $P \rightarrow B$ has an integral closed connection form, then the argument given above shows that its structural group reduces to $\operatorname{Ham}^{s \mathbb{Z}}$. Conversely, Lemma 3.2 shows that any bundle pulled back from $B \mathrm{Ham}^{s \mathbb{Z}}$ has a closed connection form $\Omega$ that takes integral values on cycles lying over $B_{1}$. In other words, $[\Omega]$ takes integral values on the elements in $\operatorname{ker} \pi_{*}: H_{2}(P ; \mathbb{Z}) \rightarrow H_{2}(B ; \mathbb{Z})$. Hence, the homomorphism $H_{2}(P ; \mathbb{Z}) \rightarrow \mathbb{R} / \mathbb{Z}$ induced by $[\Omega]$ may be written as a composite $f \circ \pi_{*}$, where $f: H_{2}(B ; \mathbb{Z}) \rightarrow$ $\mathbb{R} / \mathbb{Z}$. It suffices to check that $f$ lifts to a homomorphism $\beta: H_{2}(B ; \mathbb{Z}) \rightarrow \mathbb{R}$. Thus we need $f$ to vanish on the torsion elements of $H_{2}(B ; \mathbb{Z})$. This is obvious if $H_{2}(B ; \mathbb{Z})$ is free, while, if there is a section over the 3 -skeleton, $f$ vanishes on the torsion classes in $B$ because they lift to torsion classes in $P$.

We show in the next section that part (i) of this proposition extends to arbitrary bundles. However, the integral case is more subtle. Example 4.2 shows that when $[\omega]$ is integral, there might be manifolds $(M, \omega)$ for which there is no group that classifies symplectic $M$-bundles with integral closed connection form. Moreover, the following example shows that the obstruction to the existence of an integral extension of $[\omega]$ does involve the 3 -skeleton of $B$. Take the universal $S^{2}$-bundle $P \rightarrow B S O(3)$ and provide $S^{2}$ with a symplectic form $\omega$ in the class that generates $H^{2}\left(S^{2} ; \mathbb{Z}\right)$. Then the first Chern class $c:=c_{1}^{\text {Vert }}$ of the vertical tangent bundle extends $2[\omega]$. But $\omega$ has no integral extension; $c / 2$ is not integral (the restriction of $P$ over the 2-skeleton is the one-point blow up of $\left.\mathbb{C} P^{2}\right)$ and $[\omega]$ has a unique extension because $H^{2}(B \mathrm{SO}(3) ; \mathbb{Z})=0$.

3.2. The obstruction class. Let $\Lambda$ be a countable subgroup of $H^{1}(M ; \mathbb{R})$ that contains $\Gamma$ and is invariant under the action of $\pi_{0}$ (Symp). Denote by $\mathcal{A}_{\Lambda}$ the quasitopological Abelian group $H^{1}(M ; \mathbb{R}) / \Lambda$. As explained in Remark 2.6, $\mathcal{A}_{\Lambda}$ is homotopy equivalent to a $K(\pi, 1)$ with $\pi_{1}$ isomorphic to the free Abelian group $\Lambda$. Let $\widehat{F}_{\Lambda}:$ Symp $\rightarrow \mathcal{A}_{\Lambda}$ be a crossed homomorphism whose restriction to $\operatorname{Symp}_{0}$ factors through Flux. Denote its kernel by $\mathcal{H}_{\Lambda}$. The next lemmas hold trivially when $\Lambda=0$, for in this case the inclusion $\mathcal{H}_{\Lambda} \rightarrow$ Symp is a homotopy equivalence. 
Lemma 3.4. There is an obstruction class

$$
\mathcal{O}_{\Lambda}^{M} \in H^{2}(B \text { Symp; } \Lambda),
$$

such that the classifying map $\phi: B \rightarrow B$ Symp of a symplectic bundle lifts to $B \mathcal{H}_{\Lambda}$ iff $\phi^{*}\left(\mathcal{O}_{\Lambda}^{M}\right)=0$.

Proof. Suppose first that $\Lambda$ is a discrete subgroup of $H^{1}(M ; \mathbb{R})$ so that $\mathcal{A}_{\Lambda}$ is a topological (rather than quasitopological) space. Consider the fibration sequence

$$
\mathcal{H}_{\Lambda} \longrightarrow \operatorname{Symp} \stackrel{\widehat{F}_{\Lambda}}{\longrightarrow} \mathcal{A}_{\Lambda},
$$

that identifies $\mathcal{A}_{\Lambda}$ as the homogeneous space Symp $/ \mathcal{H}_{\Lambda}$. There is an associated homotopy fibration

$$
\mathcal{A}_{\Lambda} \rightarrow B \mathcal{H}_{\Lambda} \longrightarrow B \text { Symp. }
$$

Because $\mathcal{A}_{\Lambda}$ is a $K(\pi, 1)$, there is a single obstruction to the existence of a section of this fibration, namely a class $\mathcal{O}_{\Lambda}^{M} \in H^{2}\left(B \operatorname{Symp} ; \pi_{1}\left(\mathcal{A}_{\Lambda}\right)\right)=$ $H^{2}(B$ Symp; $\Lambda)$.

In the general case, we should replace $\mathcal{A}$ by the homotopy quotient Symp $/ / \mathcal{H}_{\Lambda}$ and consider the homotopy fibration

$$
\text { Symp } / / \mathcal{H}_{\Lambda} \rightarrow \operatorname{Symp}\left\|\operatorname{Symp} / / \mathcal{H}_{\Lambda} \rightarrow \operatorname{Symp}\right\| *,
$$

where $*$ denotes the one point space, and the notation $G \rrbracket X / / H$ denotes the realization of the topological category formed from the action of the group $G \times H$ on $X$, where $G$ acts by multiplication on the left and $H$ by multiplication on the right. As explained in [19], Symp $\$ Symp is contractible, and so Symp $\| \operatorname{Symp} / / \mathcal{H}_{\Lambda}=(\operatorname{Symp} \| \operatorname{Symp}) / / \mathcal{H}_{\Lambda}$ is homotopy equivalent to $B \mathcal{H}_{\Lambda}$. Hence the above fibration is a model for $\mathcal{A}_{\Lambda} \rightarrow B \mathcal{H}_{\Lambda} \rightarrow B$ Symp, and the argument proceeds as before.

We now suppose that $\Lambda=H^{1}\left(M ; \Lambda^{\prime}\right)$, where $\Lambda^{\prime}$ is some countable subgroup of $\mathbb{R}$ containing the integral periods $\mathcal{P}_{\omega}^{\mathbb{Z}}$ of $[\omega]$. Thus $[\omega] \in H^{2}\left(M ; \Lambda^{\prime}\right)$. In the next lemma

$$
d_{2}^{\Lambda}: H^{2}\left(M ; \Lambda^{\prime}\right) \rightarrow H^{2}\left(B \text { Symp; } H^{1}\left(M ; \Lambda^{\prime}\right)\right)
$$

denotes the second differential in the Leray-Serre spectral sequence for the cohomology of $M_{\text {Symp }} \rightarrow B$ Symp with coefficients $\Lambda^{\prime}$.

Lemma 3.5. $\mathcal{O}_{\Lambda}^{M}=d_{2}^{\Lambda}([\omega])$.

Proof. Give $B:=B$ Symp a CW decomposition with one vertex $*$ and fix an identification of the fiber $M_{*}$ over this vertex with $M$. We shall show that both $\mathcal{O}_{\Lambda}^{M}$ and $d_{2}^{\Lambda}([\omega])$ may be interpreted as the first obstruction to defining a closed connection form $\Omega$ over the 2-skeleton $B_{2}$ of $B$ whose monodromy round the loops in $B_{1}$ lies in $\mathcal{H}_{\Lambda}$. Note that because every loop in $B$ is 
homotopic to one in $B_{1}$ and because Ham $\subset \mathcal{H}_{\Lambda}$, any such connection does have monodromy in $\mathcal{H}_{\Lambda}$ and hence does define an $\mathcal{H}_{\Lambda}$-structure.

Choose a closed extension $\Omega^{\prime}$ of $\omega$ over the 1 -skeleton $B_{1}$ of $B$. Since $\mathcal{H}_{\Lambda}$ intersects every component of Symp, we may suppose that the holonomy of $\Omega^{\prime}$ is contained in $\mathcal{H}_{\Lambda}$. Let $\alpha: D \rightarrow B_{2}$ be a 2-cell attached via $\alpha: \partial D \rightarrow B_{1}$. Choose an identification $\Psi$ of the pullback symplectic bundle $\pi_{D}: \alpha^{*}\left(M_{\text {Symp }}\right) \rightarrow D$ with the product $D \times M \rightarrow D$ that extends the given identification $M_{*}=M$. Then $\Psi$ is well defined modulo diffeomorphisms of $D \times M$ of the form $(z, x) \mapsto\left(z, \psi_{z}(x)\right)$, where $\psi_{z} \in \operatorname{Symp}_{0}, \psi_{*}=$ id. Hence the induced identification of $H^{2}\left(\pi_{D}^{-1}(\partial D)\right)$ with $H^{2}\left(S^{1} \times M ; \Lambda\right)=H^{2}(M ; \Lambda) \oplus H^{1}\left(S^{1} ; \mathbb{Z}\right) \otimes H^{1}(M ; \Lambda)$ is independent of choices.

Consider the pullback $\Omega_{D}^{\prime}$ of $\Omega^{\prime}$ to $\partial D \times M$. Let $h_{t}, t \in[0,1]$ be the family of symplectomorphisms defining its characteristic flow, i.e., for each $x$, the paths $\left(t, h_{t}(x)\right)$ lie in the null space of $\Omega_{D}^{\prime}$. Then $\Omega_{D}^{\prime}$ represents the class $[\omega]+[d t] \times a_{D}$ in $H^{2}\left(S^{1} \times M ; \Lambda\right)$, where $a_{D}=\operatorname{Flux}\left(\left\{h_{t}\right\}\right)$. Since $h_{1} \in \mathcal{H}_{\Lambda}$ by construction, $a_{D} \in \Lambda:=H^{1}\left(M ; \Lambda^{\prime}\right)$. Note that $\Omega_{D}^{\prime}$ extends to a closed form over $\pi_{D}^{-1}(D)$ iff $a_{D}=0$. Hence the cocycle $D \mapsto a_{D}$ represents the obstruction class $\mathcal{O}_{\Lambda}^{M}$. It is also clear from the interpretation of $d_{2}$ via zigzags given in Bott-Tu [2, Theorem 14.14] that this cocycle also represents $d_{2}^{\Lambda}([\omega])$. The fact that we only consider forms $\Omega^{\prime}$ over $B_{1}$ with monodromy in $\mathcal{H}_{\Lambda}$ corresponds to the fact that we restrict the coefficients to $\Lambda^{\prime}$.

Corollary 3.6. Suppose that $\Lambda^{\prime}$ is divisible, i.e., is a module over $\mathbb{Q}$. Then the group $\mathcal{H}_{\Lambda}$ has the extension property.

Proof. Since $\Lambda^{\prime}$ is divisible, $d_{2}^{\Lambda}([\omega])=0$ iff $d_{2}([\omega])=0$ in the spectral sequence with coefficients $\mathbb{R}$. Since $d_{3}^{\Lambda}([\omega])$ also vanishes by Lemma 1.6, it follows that a symplectic bundle has a $\mathcal{H}_{\Lambda}$-structure iff it has a closed connection form.

In particular, this proves Corollary 1.11. We now prove the other statements in Section 1.2. When $\Lambda^{\prime}=\mathcal{P}_{\omega}$, the rational period group of $[\omega]$, we denote $\mathcal{O}_{\Lambda}^{M}=: \mathcal{O}^{M}$.

Proof of Theorem 1.10. Part (i) is an immediate consequence of Lemma 3.5. Now suppose there is an extension $\widetilde{F}: \operatorname{Symp} \rightarrow H^{1}(M ; \mathbb{R}) / \Gamma$ of Flux. The proof of Lemma 3.5 shows that the image of $\mathcal{O}_{\Gamma}^{M} \in H^{2}(B$ Symp; $\Gamma$ ) in $H^{2}\left(B\right.$ Symp; $\left.H^{1}\left(M ; \mathcal{P}_{\omega}\right)\right)$ is $d_{2}^{\omega}([\omega])=\mathcal{O}^{M}$. Thus $\mathcal{O}^{M}$ takes values in $\Gamma$. Hence, it remains to prove the converse, i.e., that Flux extends if $\mathcal{O}^{M}$ takes values in $\Gamma$.

As in the proof of Proposition 1.8 given in Section 1, it suffices to find a section

$$
\sigma: \pi_{0}(\mathrm{Symp}) \longrightarrow \text { Symp, } \quad \alpha \longmapsto \sigma_{\alpha} \in \operatorname{Symp}_{\alpha}
$$


such that

$$
\left(\sigma_{\alpha \beta}\right)^{-1} \sigma_{\alpha} \sigma_{\beta} \in \text { Ham, } \quad \alpha, \beta \in \pi_{0}(\text { Symp }) .
$$

To do this, consider the fibration sequence $\mathcal{A} \rightarrow B$ Ham $^{s} \stackrel{\pi}{\rightarrow} B$ Symp of Lemma 3.4. By assumption, the obstruction to the existence of a section $s: B$ Symp $\rightarrow B \mathrm{Ham}^{s}$ is an element of $H^{2}(B \mathrm{Symp} ; \Gamma)$, where $\Gamma$ is identified with its image in $H_{\mathbb{Q}}=\pi_{1}(\mathcal{A})$. This means that for any compatible $\mathrm{CW}$ structures put on $B \mathrm{Ham}^{s}$ and $B$ Symp, one can choose a map $s:(B \text { Symp })_{1} \rightarrow\left(B \operatorname{Ham}^{s}\right)_{1}$, (where $B_{1}$ denotes the 1-skeleton of $\left.B\right)$ so that $\pi \circ s \sim$ id and so that the corresponding obstruction cocycle takes values in $\Gamma$. Choose a CW structure on $B$ Symp with one vertex, and one 1-cell $I \times g_{\alpha}$ for each component $\alpha \in \pi_{0}$ (Symp). (This is possible because $\pi_{0}$ (Symp) is countable.) Then for each pair $\alpha, \beta$ in $\pi_{0}$ (Symp), there is a 2-cell $c_{\alpha, \beta}$ with boundary $\left(I \times g_{\alpha \beta}\right)^{-1}\left(I \times g_{\alpha}\right)\left(I \times g_{\beta}\right)$. (There are other 2-cells in $B$ Symp coming from the 1-skeleton of a CW decomposition for Symp, but these are irrelevant for the current argument.) We define a CW structure on $B \mathrm{Ham}^{s}$ in a similar way. Then the map $s$ takes each 1-cell $I \times g_{\alpha}$ in $B$ Symp to a loop in $\left(B \mathrm{Ham}^{s}\right)_{1}$. This loop is given by a word $w_{\alpha}$ in the elements of $\mathrm{Ham}^{s}$ that represents an element $h\left(w_{\alpha}\right)$ in $\operatorname{Ham}^{s} \cap \operatorname{Symp}_{\alpha}$.

The obstruction to extending $s$ over the 2-cell $c_{\alpha, \beta}$ is the homotopy class in $B \mathrm{Ham}^{s}$ of the loop corresponding to the word $\left(w_{\alpha \beta}\right)^{-1} w_{\alpha} w_{\beta}$. This can be identified with the homotopy class

$$
\left[h\left(w_{\alpha \beta}\right)^{-1} h\left(w_{\alpha}\right) h\left(w_{\beta}\right)\right] \in \pi_{0}\left(\operatorname{Ham}^{s} \cap \operatorname{Symp}_{0}\right) \cong H^{1}\left(M ; \mathcal{P}_{\omega}\right) / \Gamma
$$

of the element $h\left(w_{\alpha \beta}\right)^{-1} h\left(w_{\alpha}\right) h\left(w_{\beta}\right)$. To say the obstruction $\mathcal{O}^{M}\left(c_{\alpha, \beta}\right)$ takes values in $\Gamma$ means that this class lies in $\pi_{0}\left(\operatorname{Ham}^{s}\right)=$ Ham. Hence, it is always possible to extend $s$ over these 2-cells (though it may not extend over the other 2-cells in $B$ Symp). Further, if we define the section $\sigma: \pi_{0}($ Symp $) \rightarrow$ Symp by $\sigma(\alpha):=h\left(w_{\alpha}\right)$, then identity (3.1) holds. This completes the proof.

Remark 3.7. We sketch an alternative way to prove this result based on the ideas in Kędra-Kotschick-Morita [6]. Suppose that $\mathcal{O}^{M}$ takes values in $\Gamma$ but does not vanish on the 2 -skeleton $B_{2}$ of $B$ Symp. Then one can form a new bundle $P_{2}^{\prime} \rightarrow B_{2}$ with vanishing obstruction class by appropriately twisting the given bundle over each 2-cell $c$ in $B_{2}$ for which $\mathcal{O}^{M}(c) \neq 0$; for each such $c$ change the bundle by taking the connect sum with an appropriate bundle $Q \rightarrow S^{2}$. By construction, there is a closed connection form $\Omega^{\prime}$ on $P_{2}^{\prime}$. Since we did not change the bundle over $B_{1}$, the construction in $[6$, Theorem 6] gives a class $[F] \in H^{1}\left(\left(B \operatorname{Symp}^{\delta}\right)_{1} ; H^{1}(M ; \mathbb{R})\right)$. (As described in Section 4.2 , this is induced by the difference $[\widetilde{\omega}]-\iota^{*}\left[\Omega^{\prime}\right] \in H^{2}\left(\iota^{*}\left(P_{1}\right) ; \mathbb{R}\right)$, where $\iota: B$ Symp $^{\delta} \rightarrow B$ Symp.) This class $[F]$ is represented by an EilenbergMacLane cochain on the group $\operatorname{Symp}^{\delta}$ with values in $H^{1}(M ; \mathbb{R})$. However, 
because we changed the bundle over the 2-cells, it satisfies the cocycle condition only when projected to the quotient $H^{1}(M ; \mathbb{R}) / \Gamma$. Hence, it gives rise to a crossed homomorphism Symp $\rightarrow H^{1}(M ; \mathbb{R}) / \Gamma$ that extends Flux.

We next turn to the proof of Proposition 1.13 which we restate for the convenience of the reader. Since (ii) and (iii) are obviously equivalent, we omit (ii) here.

Lemma 3.8. The following conditions are equivalent.

(i) There is an extension $\widetilde{F}: \operatorname{Symp}(M, \omega) \rightarrow H^{1}(M ; \mathbb{R}) / \Gamma$ of Flux.

(ii) For every product of commutators $\left[u_{1}, u_{2}\right] \ldots\left[u_{2 p-1}, u_{2 p}\right], u_{i} \in$ Symp, that lies in $\mathrm{Symp}_{0}$, there are elements $g_{1}, \ldots, g_{2 p} \in \mathrm{Symp}_{0}$ such that

$$
\left[u_{1} g_{1}, u_{2} g_{2}\right] \ldots\left[u_{2 p-1} g_{2 p-1}, u_{2 p} g_{2 p}\right] \in \text { Ham }
$$

(iii) For every symplectic $M$-bundle $P \rightarrow \Sigma$, there is a bundle $Q \rightarrow S^{2}$ such that the fiberwise connect sum $P \# Q \rightarrow \Sigma \# S^{2}=\Sigma$ has a closed connection form.

Proof. Because $\mathcal{O}^{M} \in H^{2}\left(B\right.$ Symp; $\left.H^{1}\left(M ; \mathcal{P}_{\omega}\right)\right)$ where $\mathcal{P}_{\omega}$ is divisible, $\Omega$ lies in the image of $H^{2}(B$ Symp; $\Gamma)$ iff its pullback over every map $\phi: \Sigma \rightarrow$ $B$ Symp takes values in $\Gamma$. Since $\Gamma$ is the image of $\pi_{1}$ (Symp) under the Flux homomorphism, for every element $\gamma \in \Gamma$, there is a corresponding $M$-bundle $Q \rightarrow S^{2}$ which is classified by a map $\phi: S^{2} \rightarrow B$ Symp such that $\phi^{*}\left(\mathcal{O}^{M}\right)\left(\left[S^{2}\right]\right)=\gamma$. Further, if $\phi: \Sigma \rightarrow B$ Symp classifies the bundle $P \rightarrow \Sigma$ and $\psi: S^{2} \rightarrow B$ Symp classifies $Q \rightarrow S^{2}$, the fiber connect sum $P \# Q \rightarrow \Sigma$ is classified by $\phi \vee \psi: \Sigma \vee S^{2} \rightarrow B$ Symp. Therefore, the equivalence of (i) and (iii) follows immediately from Theorem 1.10(ii).

To see that (i) implies (ii) observe that if we write $\mathcal{H}:=\operatorname{ker} \widetilde{F}$, then the quotient group $\mathcal{H} /$ Ham is isomorphic to $\pi_{0}\left(\right.$ Symp) because $\mathcal{H} \cap \mathrm{Symp}_{0}=$ Ham. Hence, any representation in $\pi_{0}$ (Symp) can be lifted to the subgroup $\mathcal{H}$ / Ham of Symp / Ham. This immediately implies (ii).

It remains to show that (ii) implies (iii), which we do by direct construction. Consider any symplectic bundle $\pi: P \rightarrow \Sigma_{g}$. Decompose it into the union of a trivial bundle $M \times D \rightarrow D$ over a 2-disc with a symplectic bundle $P^{\prime} \rightarrow \Sigma_{g} \backslash D$. Choose a flat symplectic connection $\Omega^{\prime}$ on $P^{\prime}$ whose holonomy round the generators of $\pi_{1}\left(\Sigma_{g}\right)$ is given by elements $u_{i} \in$ Symp. Since the symplectic trivialization of $\pi$ over $D$ is determined up to a Hamiltonian isotopy, there is an identification of $\partial P^{\prime}$ with $M \times S^{1}$ that is well defined up to a Hamiltonian loop $g_{t} \in \operatorname{Ham}, t \in \mathbb{R} / \mathbb{Z}$. Thus the holonomy of $\Omega^{\prime}$ round $\partial P^{\prime}$ gives rise to a path $h_{t}$ from the identity to $f:=\left[u_{1}, u_{2}\right] \cdots\left[u_{2 g-1}, u_{2 g}\right]$ that is well defined modulo a Hamiltonian loop. Hence, $\operatorname{Flux}\left(\left\{h_{t}\right\}\right) \in H^{1}(M ; \mathbb{R})$ depends only on the choice of flat connection $\Omega^{\prime}$. By (ii) we may homotop 
the $u_{i}$ (or equivalently choose $\Omega^{\prime}$ ) so that $f \in$ Ham. Thus the obstruction $\phi^{*}\left(\mathcal{O}^{M}\right)([\Sigma])=\operatorname{Flux}\left(\left\{h_{t}\right\}\right)$ lies in $\Gamma$.

Proof of Proposition 1.3. Let $\mathcal{H}$ be a subgroup of Symp with identity component Ham and consider the image $\operatorname{Im}\left(\pi_{0}(\mathcal{H})\right)$ of $\pi_{0}(\mathcal{H})$ in $\pi_{0}(\operatorname{Symp})$. If $\mathcal{H}$ has the modified extension property, then we must show that the intersection with $\operatorname{Im}\left(\pi_{0}(\mathcal{H})\right)$ of every finitely generated subgroup $G$ of $\pi_{0}(\operatorname{Symp})$ has finite index in $G$. But otherwise there would be a map of a finite wedge $V$ of circles into $B\left(\pi_{0}(\mathrm{Symp})\right)$ such that no finite cover $\widetilde{V}$ of $V$ lifts into the image of $B \mathcal{H}$ in $B\left(\pi_{0}(\right.$ Symp $\left.)\right)$. Since any bundle over a 1-complex has a closed extension form, this contradicts our assumption on $\mathcal{H}$.

Conversely, assume that the cokernel of $\operatorname{Im}\left(\pi_{0}(\mathcal{H})\right)$ in $\pi_{0}(\mathrm{Symp})$ has the stated finiteness properties and let $\operatorname{Symp}_{\mathcal{H}}$ be the subgroup of Symp consisting of elements isotopic to $\mathcal{H}$. If $P \rightarrow B$ is classified by a map into $B \mathcal{H}$, then it has a closed connection form by Corollary 3.1. Therefore, we just need to see that if $\phi: B \rightarrow B$ Symp classifies a bundle with a closed extension form its pullback over some finite cover $\widetilde{B} \rightarrow B$ lifts to $B \mathcal{H}$.

Observe first that the composite map $\pi_{1}(B) \rightarrow \pi_{0}(\operatorname{Symp}) / \pi_{0}\left(\operatorname{Symp}_{\mathcal{H}}\right)$ has finite image by hypothesis. (Recall that we always assume $\pi_{1}(B)$ is finitely generated.) Therefore, we may replace $B$ by a finite cover such that the pullback bundle $\widetilde{P} \rightarrow \widetilde{B}$ is classified by a map $\widetilde{\phi}: \widetilde{B} \rightarrow B \operatorname{Symp}_{\mathcal{H}}$. Set $\Lambda \subset H^{1}(M ; \mathbb{R})$ equal to the (discrete) $\operatorname{group} \operatorname{Flux}\left(\mathcal{H} \cap \operatorname{Symp}_{0}\right)$ and then define a crossed homomorphism

$$
F: \operatorname{Symp}_{\mathcal{H}} \longrightarrow H^{1}(M ; \mathbb{R}) / \Lambda
$$

as in the proof of Proposition 1.8 given in Section 1.3. Because the bundle $\widetilde{P} \rightarrow \widetilde{B}$ has a closed connection form, the class $\widetilde{\phi}^{*}\left(d_{2}([\omega])\right.$ vanishes in $H^{2}\left(\widetilde{B} ; H^{1}(M ; \mathbb{R})\right)$. The proof of Lemma 3.5 shows that this class is the image of $\mathcal{O}_{\Lambda}^{M} \in H^{2}(\widetilde{B} ; \Lambda)$ under the map induced by the inclusion $\Lambda \rightarrow H^{1}(M ; \mathbb{R})$. By pulling back over a further cover if necessary, we may suppose that $H^{2}(\widetilde{B} ; \Lambda)$ has no torsion. (Since $\pi_{1}(B)$ may act nontrivially on the coefficients $\Lambda$, it is not enough to assume that $H_{1}(\widetilde{B} ; \mathbb{Z})$ is free.) Hence, this map is injective and $\mathcal{O}_{\Lambda}^{M}=0$. Thus $\widetilde{\phi}$ lifts to $B \mathcal{H}$ as required.

Proof of Proposition 1.4. The first claim is that Ham has the modified restricted extension property. This is a corrected statement of the conclusions that one can draw from the proof of Theorem 1.1 in [11]. The claim also follows by arguing as in the proof of Proposition 1.3 using Flux instead of $\widehat{F}_{s}$; the argument can be greatly simplified because the group $\mathrm{Symp}_{0}$ acts trivially on the coefficients. Here one should also note that if the cover $\widetilde{B} \rightarrow B$ is chosen so that $H_{1}(\widetilde{B} ; \mathbb{Z})$ has no torsion, then the boundary map $\delta: H^{1}\left(B ; H_{\mathbb{R}} / H_{\mathbb{Q}}\right) \rightarrow H^{2}\left(B ; H_{\mathbb{Q}}\right)$ vanishes. 
The second claim is that when $\Gamma \neq 0$, the group Ham does not have the extension property. To see this, choose a nonzero element $\beta \in H^{1}(M ; \mathbb{R}) \backslash \Gamma$ such that $2 \beta \in \Gamma$ and then choose $g \in \operatorname{Symp}_{0}$ with $\operatorname{Flux}(g)=\beta$. Consider the bundle $P \rightarrow \mathbb{R} P^{2}$ that is formed from the mapping torus bundle

$$
M_{g}:=M \times[0,1] /((x, 1) \sim(g x, 0)) \longrightarrow S^{1}
$$

by attaching $M \times D^{2}$ by the map $\left(x, e^{2 \pi i t}\right) \mapsto\left(g_{t}(x), 2 t\right)$, where $g_{t}$ is a path in Ham from the identity to $g_{1}:=g^{-2} \in$ Ham. The flat connection on $M_{g}$ pulls back to a connection with Hamiltonian monodromy round the boundary $M \times \partial D$ and so extends to a closed connection form over the rest of $P$ : cf. the proof of Lemma 3.5.

We claim that this bundle has no Hamiltonian structure. To see this, consider the classifying map $\phi: \mathbb{R} P^{2} \rightarrow B$ Symp $_{0}$. Just as in the discussion before Lemma 3.4, the homomorphism Flux : $\operatorname{Symp}_{0} \rightarrow H_{\mathbb{R}} / \Gamma$ defines an obstruction class $\mathcal{O}_{\Gamma}^{M} \in H^{2}\left(B \operatorname{Symp}_{0} ; \Gamma\right)$ such that $\phi^{*}\left(\mathcal{O}_{\Gamma}^{M}\right)$ vanishes iff the bundle $P \rightarrow \mathbb{R} P^{2}$ has a Hamiltonian structure. Since $B\left(H_{\mathbb{R}} / \Gamma\right)$ is a $K(\Gamma, 2)$, this class is the pullback to $B$ Symp $_{0}$ of the canonical generator of $H^{2}(K(\Gamma, 2) ; \Gamma)$. We claim that the composite map

$$
\mathbb{R} P^{2} \rightarrow B \mathrm{Symp}_{0} \rightarrow B\left(H_{\mathbb{R}} / \Gamma\right)=K(\Gamma, 2)
$$

is not null homotopic. Since $\mathbb{R} P^{2}$ is the 2 -skeleton of $\mathbb{R} P^{\infty}=K(\mathbb{Z} / 2 \mathbb{Z} ; 1)$ and $K(\Gamma, 2)$ is homotopy equivalent to a product of copies of $B S^{1}$, this assertion is equivalent to saying that under the map $B(\mathbb{Z} / 2 \mathbb{Z}) \rightarrow B S^{1}$ induced by the obvious inclusion $\{ \pm 1\} \rightarrow S^{1}$ the generator of $H^{2}\left(B S^{1} ; \mathbb{Z} / 2 \mathbb{Z}\right)$ pulls back to a nonzero element of $H^{2}\left(\mathbb{R} P^{2} ; \mathbb{Z} / 2 \mathbb{Z}\right)$. This is well known. For a direct proof, identify the 2 -skeleton of $B S^{1}=\mathbb{C} P^{\infty}$ with the quotient $S^{3} / S^{1}$ and observe that the $\mathbb{Z} / 2 \mathbb{Z}$-equivariant map

$$
S^{2} \longrightarrow S^{3}, \quad(r, s, t) \longmapsto\left(r+i s, \frac{1}{\sqrt{2}}(t+i t)\right) \in S^{3} \subset \mathbb{C}^{2},
$$

descends to a map $\mathbb{R} P^{2} \rightarrow S^{2}$ of $(\bmod 2)$ degree 1 .

3.3. Stability. We finally discuss the question of stability.

Proof of Proposition 1.15. Let $\mathcal{N}(\omega)$ be a path connected neighborhood of $\omega$ in the space of forms annihilating $V_{2}(P)$ such that $P \rightarrow B$ has an $\omega^{\prime}$-symplectic structure for all $\omega^{\prime} \in \mathcal{N}(\omega)$. Our aim is to shrink $\mathcal{N}(\omega)$ so that each such $\omega^{\prime}$ has a closed extension to $P$. We claim that for each map $\psi: \Sigma \rightarrow B$ of a Riemann surface into $B$, there is a homologous map $\psi^{\prime}: \Sigma^{\prime} \rightarrow B$ such that the pullback bundle over $\Sigma^{\prime}$ admits a closed extension of $\omega^{\prime}$, provided that $\omega^{\prime}$ is sufficiently close to $\omega$. Granted this, we may choose $\mathcal{N}(\omega)$ so that this holds for a finite set of $\psi_{i}$ that represent a set of generators for $H_{2}(M ; \mathbb{R})$ and all $\omega^{\prime} \in \mathcal{N}(\omega)$. It follows that the obstruction class $\mathcal{O}_{\omega^{\prime}}^{M}$ 
must vanish when pulled back to $B$, i.e., that $\left(M, \omega^{\prime}\right) \rightarrow P \rightarrow B$ has a closed connection form when $\omega^{\prime} \in \mathcal{N}(\omega)$.

To prove the claim, consider a map $\psi: \Sigma:=\Sigma_{p} \rightarrow B$. As in the proof of Lemma 3.8, we may assume that the pullback bundle $\psi^{*} P \rightarrow \Sigma$ has a flat $\omega$-symplectic connection over $\Sigma \backslash D^{2}$ whose holonomy $y$ around the boundary of the disc $D^{2}$ may be expressed as:

$$
y:=\left[u_{1}, u_{2}\right] \cdots\left[u_{2 p-1}, u_{2 p}\right] \in \operatorname{Ham}(M, \omega), \quad u_{i} \in \operatorname{Symp}(M, \omega) .
$$

Since $\operatorname{Ham}(M, \omega)$ is a perfect group, we may, by increasing the genus of $\Sigma$ and choosing the flat connection on the extra handles to have Hamiltonian holonomy, assume that $y=\mathrm{id}$. By hypothesis on the deformation $\omega^{\prime}$, we can choose:

- a path $\omega_{t}$ from $\omega_{0}:=\omega$ to $\omega_{1}:=\omega^{\prime}$ in $\mathcal{N}([\omega])$, and

- $C^{1}$-small paths $g_{i t} \in \operatorname{Diff}_{0}(M)$ such that $u_{i} g_{i t} \in \operatorname{Symp}\left(M, \omega_{t}\right)$ for all $i$ and $t \in[0,1]$. (These may be constructed using the Moser method.)

Since $y=\mathrm{id}$, the smooth path

$$
y_{t}=\left[u_{1} g_{1 t}, u_{2} g_{2 t}\right] \cdots\left[u_{2 p-1} g_{(2 p-1) t}, u_{2 p} g_{(2 p) t}\right]
$$

is $C^{1}$-small and lies in $\operatorname{Symp}_{0}\left(M, \omega_{t}\right)$ for all $t$. If we could arrange that $y_{t} \in \operatorname{Ham}\left(M, \omega_{t}\right)$ for each $t$, then the connection could be extended to a Hamiltonian connection over the disc for all $t$ and the proof would be complete.

We do this in two stages. First we modify $\psi: \Sigma \rightarrow B$ to a map $\psi^{\prime}: \Sigma^{\prime} \rightarrow B$ so that $y_{t} \in \operatorname{Ham}^{s}\left(M, \omega_{t}\right) \cap \operatorname{Symp}_{0}\left(M, \omega_{t}\right)$ for all $t$. To this end, consider the subspace $V^{1}$ of $H^{1}(M ; \mathbb{R})$ generated by the elements $u_{i}^{*} \alpha-\alpha$, where $i=1, \ldots, 2 p$, and $\alpha$ runs through the elements of $H^{1}(M ; \mathbb{R})$. If the elements $a, b \in \operatorname{Symp}(M, \omega)$ are each homotopic to some $u_{i}, i=1, \ldots, 2 p$ then

$$
\begin{aligned}
\widehat{F}^{s}([a, b]) & =\widehat{F}^{s}\left(b^{-1}\right)+\left(b^{-1}\right)^{*} \widehat{F}^{s}\left(a^{-1}\right)+\left(a^{-1} b^{-1}\right)^{*} \widehat{F}^{s}(b)+\left(b a^{-1} b^{-1}\right)^{*} \widehat{F}^{s}(a) \\
& =-b^{*} \widehat{F}^{s}(b)+\left(a^{-1} b^{-1}\right)^{*} \widehat{F}^{s}(b)-\left(b^{-1}\right)^{*} a^{*} \widehat{F}^{s}(a)+\left(b a^{-1} b^{-1}\right)^{*} \widehat{F}^{s}(a),
\end{aligned}
$$

which is easily seen to lie in $V^{1} /\left(V^{1} \cap H^{1}\left(M ; \mathcal{P}_{\omega}\right)\right)$. Hence,

$$
\widehat{F}_{\omega_{t}}^{s}\left(y_{t}\right) \in V^{1} /\left(V^{1} \cap H^{1}\left(M ; \mathcal{P}_{\omega_{t}}\right)\right), \quad t \in[0,1] .
$$

By compactness we can therefore find a finite collection of smooth families $\left(v_{j t}, \alpha_{j t}\right), j=1, \ldots, m$, such that

$$
\sum_{j=1}^{m} v_{j}^{*} \alpha_{j t}-\alpha_{j t} \in \widehat{F}_{\omega_{t}}^{s}\left(y_{t}\right)+H^{1}\left(M ; \mathcal{P}_{\omega_{t}}\right), \quad t \in[0,1],
$$

where each $v_{j t} \in \operatorname{Symp}\left(M, \omega_{t}\right)$ is a product of the elements $\left(u_{i} g_{i t}\right)^{ \pm 1}, i=$ $1, \ldots, 2 p$, and $\alpha_{j t}$ is a path in $H^{1}(M ; \mathbb{R})$ with initial point $\alpha_{j 0}=0$. 
For each pair $\left(v_{j t}, \alpha_{j t}\right)$, choose a path $\widetilde{h}_{j t}$ in $\widetilde{\operatorname{Symp}}_{0}\left(M, \omega_{t}\right)$ starting at id such that $\operatorname{Flux}_{\omega_{t}} \widetilde{h}_{j t}=\alpha_{j t}$. Then,

$$
\operatorname{Flux}_{\omega_{t}}\left[v_{j t}^{-1}, \widetilde{h}_{j t}^{-1}\right]=\alpha_{j t}-v_{j t}^{*} \alpha_{j t}=: \beta_{j t} .
$$

Therefore, there is a fibration $M \rightarrow Q_{j} \rightarrow \mathbb{T}^{2}$ that admits an $\omega_{t}$-symplectic structure for each $t$ and a flat connection over $\mathbb{T}^{2} \backslash D^{2}$ whose boundary holonomy has flux $\beta_{j t}$. As an $\omega_{t}$-symplectic bundle, $Q_{j}$ is pulled back from a bundle over $S^{1}$ with holonomy $v_{j t}$. Our choice of $v_{j t}$ implies this bundle is a pullback of $P \rightarrow B$ by some map $\psi_{j}: S^{1} \rightarrow B$ that we can assume to be independent of $t$ (since the holonomy $\beta_{j t}$ depends only on the homotopy class of $v_{j t}$ ). However, the connection varies smoothly with $t$. Therefore, we can change the flux $\widehat{F}_{\omega_{t}}^{s}\left(y_{t}\right)$ of the boundary $\omega_{t}$-holonomy of the chosen flat connection on $f^{*} P \rightarrow\left(\Sigma \backslash D^{2}\right)$ to $\widehat{F}_{\omega_{t}}^{s}\left(y_{t}\right)+\beta_{j t}$ by replacing $\psi: \Sigma \rightarrow B$ by the homologous map

$$
\psi \# \psi_{j}: \Sigma \# \mathbb{T}^{2} \rightarrow B
$$

Repeating this process for $j=1, \ldots, m$ allows us to perform the required modification.

Therefore, we have now arranged that $y_{t} \in \operatorname{Ham}^{s}\left(M, \omega_{t}\right) \cap \operatorname{Symp}_{0}\left(M, \omega_{t}\right)$ for all $t$. The following continuity argument shows that in fact $y_{t} \in$ $\operatorname{Ham}\left(M, \omega_{t}\right)$ for all $t$, which finishes the proof.

Observe that for each $[\ell] \in H_{1}(M)$ and $t \in[0,1]$, the number

$$
\Phi(t)[\ell]:=\int_{[0, t] \times S^{1}} \phi_{t, \ell}^{*} \omega_{t} \in \mathbb{R}
$$

where $\phi_{t, \ell}(r, s):=y_{r}(\ell(s))$, projects to $\widehat{F}_{\omega_{t}}^{s}\left(y_{t}\right)([\ell]) \in \mathbb{R} / \mathcal{P}_{\omega_{t}}$. Since, by assumption, $y_{t} \in \operatorname{Ham}^{s}\left(M, \omega_{t}\right)$, we find that $\Phi(t)[\ell] \in \mathcal{P}_{\omega_{t}}$ for all $t$. But $\Phi(t)$ varies continuously with $t$ and $\Phi(0)=0$. Hence, the fact that $V_{2}\left(\omega_{t}\right) \supseteq V_{2}(\omega)$ implies that $\Phi(t)=0$ for all $t$. It remains to check that $\Phi(t)$ projects to $\operatorname{Flux}_{\omega_{t}}\left(y_{t}\right) \in H^{1}(M ; \mathbb{R}) / \Gamma_{\omega_{t}}$ for all $t \in[0,1]$. But because the $y_{t}$ are $C^{1}$-small, for each fixed $t \in[0,1]$, the path $\left\{y_{r}\right\}_{r \in[0, t]}$ may be canonically homotoped to a path $\left\{y_{r t}^{\prime}\right\}_{r \in[0, t]}$ in $\operatorname{Ham}\left(M, \omega_{t}\right)$ by a Moser process that fixes its endpoints. Flux $\omega_{t}\left(y_{t}\right)$ is given by integrating $\omega_{t}$ over the corresponding chain $\phi_{t, \ell}^{\prime}:[0, t] \times S^{1} \rightarrow M$. Since this is homotopic to $\phi_{t, \ell}:[0, t] \times S^{1} \rightarrow M$ mod boundary, we find that $\Phi(t)=$ Flux $_{\omega_{t}}\left(y_{t}\right) \bmod \Gamma_{\omega_{t}}$, as required.

\section{Further considerations}

We begin by collecting together various observations about the groups $\operatorname{Ham}^{s \mathbb{Z}}$ in the case when $\mathcal{P}_{\omega}^{\mathbb{Z}}=\mathbb{Z}$. We then explain some situations in which $\widehat{F}_{s}$ lifts to a crossed homomorphism with values in $H^{1}(M ; \mathbb{R}) / \Gamma$. This is followed by a short discussion of $c$-Hamiltonian bundles and covering groups. 
4.1. The integral case. We shall assume throughout this section that $\mathcal{P}_{\omega}^{\mathbb{Z}}=\mathbb{Z}$. Many (but not all) of our results have some analog in the general case.

We begin by considering the integral analog of Lemma 2.5. If $H_{1}(M ; \mathbb{Z})$ is torsion free, then Lemma 2.2 applies and the whole of this lemma extends. But if this group has torsion, then it is possible that (ii) does not hold.

Lemma 4.1. Suppose that $\mathcal{P}_{\omega}^{\mathbb{Z}}=\mathbb{Z}$ and set Tor $:=\operatorname{Tor}\left(H_{1}(M ; \mathbb{Z})\right)$. Then: (i) $\widehat{F}_{s}^{\mathbb{Z}}$ induces a crossed homomorphism $C_{s}: \pi_{0}(\mathrm{Symp}) \rightarrow \operatorname{Hom}(\mathrm{Tor}, \mathbb{R} / \mathbb{Z})$, whose kernel equals the image of $\pi_{0}\left(\mathrm{Ham}^{s \mathbb{Z}}\right)$ in $\pi_{0}(\mathrm{Symp})$.

(ii) The image $\left[C_{s}\right]$ of $C_{s}$ in $H_{\mathrm{EM}}^{1}($ Tor $; \mathbb{R} / \mathbb{Z})$ is independent of the choice of $s$. In particular, if Symp acts trivially on Tor, then the kernel of $C_{s}$ is independent of the choice of splitting s.

(iii) There is a splitting such that $C_{s}=0$ iff $\left[C_{s}\right]=0$.

Proof. We saw in Lemma 2.5 that if $[\ell]$ has order $N$, there are precisely $N$ distinct elements of order $N$ in the coset $\pi_{\mathbb{Z}}^{-1}([\ell])$, namely $\langle\ell\rangle-\left\langle\gamma_{(i+\mu) / N}\right\rangle$ for $i=0, \ldots, N-1$, where $\mu$ is the area of a chain $W$ that bounds $N \ell$. Since $\mathrm{Symp}_{0}$ is a connected group, it must act trivially on these elements. Therefore, for each $g \in$ Symp, the restriction of $\widehat{F}_{s}^{\mathbb{Z}}(g)$ to the torsion elements in $H_{1}(M ; \mathbb{Z})$ depends only on the image of $g$ in $\pi_{0}$ (Symp). This shows that $C_{s}$ exists. Its kernel obviously contains the image of $\mathrm{Ham}^{s \mathbb{Z}}$. To complete the proof of (i) we must show that if $\widehat{F}_{s}^{\mathbb{Z}}(g)$ vanishes on Tor, then $g$ may be isotoped to an element in $\operatorname{Ham}^{s \mathbb{Z}}$. But this holds by the proof of Lemma 2.2. (Note that we may assume that $\operatorname{dim} M>2$ here since otherwise Tor $=0$.)

Statement (ii) holds by the argument of Proposition 2.3: given a splitting $s$ of $\pi_{\mathbb{Z}}$ over Tor, any other splitting $s^{\prime}$ : Tor $\rightarrow \pi_{\mathbb{Z}}^{-1}$ (Tor) has the form $s+\left\langle\gamma_{\beta([\ell])}\right\rangle$, where $\beta \in \operatorname{Hom}($ Tor; $\mathbb{R} / \mathbb{Z}$ ). (iii) is an immediate consequence of (ii).

Example 4.2. (i) We again assume that $\mathcal{P}_{\omega}^{\mathbb{Z}}=\mathbb{Z}$. Suppose that for some $g \in$ Symp, there is a loop $\ell$ such that $[\ell]$ has order $N>1$ in Tor and there is a 2-chain $W$ with boundary $g(\ell)-\ell$ and area $i / N$, where $0<i<N$. Then $C_{s}(g) \neq 0$ for all splittings $s$, and there is no splitting such that $g$ is isotopic to an element in $\mathrm{Ham}^{s \mathbb{Z}}$. Note that the corresponding mapping torus bundle

$$
M_{g}:=M \times[0,1] /((x, 1) \sim(g x, 0)) \longrightarrow S^{1}
$$

has a closed connection form but not one that is integral. ${ }^{8}$ Equivalently, $g$ does not fix any integral lift $\tau \in H^{2}(M ; \mathbb{Z})$ of $[\omega]$.

\footnotetext{
${ }^{8}$ This example is very similar to that in the proof of Proposition 1.4. But now $[\ell] \in$ $H_{1}(M ; \mathbb{Z})$ is a torsion element, and $g$ is not isotopic to the identity, though it acts trivially on $[\ell]$.
} 
(ii) The following yet more intriguing situation cannot be ruled out in any obvious way. Suppose that Tor $=\mathbb{Z} / 2 \oplus \mathbb{Z} / 2$ is generated by the elements $[\ell]$ and $\left[\ell^{\prime}\right]$ which are interchanged by two symplectomorphisms $h_{1}, h_{2}$. Suppose further that

$$
\left(h_{1}\right)_{*}\langle\ell\rangle \neq\left(h_{2}\right)_{*}\langle\ell\rangle, \quad\left(h_{1}^{2}\right)_{*}\langle\ell\rangle=\left(h_{2}^{2}\right)_{*}\langle\ell\rangle=\langle\ell\rangle .
$$

Then $g:=h_{1} h_{2}$ fixes $[\ell]$ but acts nontrivially on $\langle\ell\rangle$ and so has the properties assumed in (i) above. Now consider the splittings $s_{i}$ defined by

$$
s_{i}[\ell]=\langle\ell\rangle, \quad s_{i}\left[\ell^{\prime}\right]=\left\langle h_{i}(\ell)\right\rangle, \quad i=1,2 .
$$

Then $h_{i} \in \operatorname{Ham}^{s_{i} \mathbb{Z}}$ by construction. The corresponding mapping tori $M_{h_{i}}$

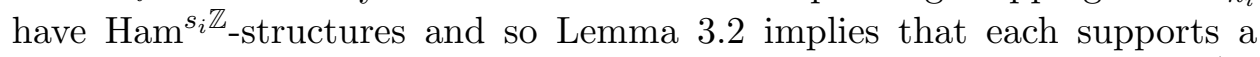
closed integral connection form. But their fiber connect sum $P \rightarrow V:=S^{1} \vee$ $S^{1}$ does not, since one of its pullbacks is the bundle $M_{g} \rightarrow S^{1}$ considered in (i). Indeed, the torsion in $H_{1}(M ; \mathbb{Z})$ creates new terms in $H_{2}(P ; \mathbb{Z})$ on which any closed connection form is nonintegral. But if every $M$-bundle with closed integral connection form is pulled back from some universal bundle $\mathcal{P} \rightarrow \mathcal{B}$ with this property, then $P \rightarrow V$ would also be such a pullback, and hence would also have a closed integral connection form. Thus, if $\operatorname{Symp}(M, \omega)$ contains elements $h_{1}, h_{2}$ as above, there is no universal $M$-bundle $\mathcal{P} \rightarrow \mathcal{B}$ with closed integral connection form.

A similar argument applies whenever there are two splittings $s_{i}, i=1,2$, such that the images of $\mathrm{Ham}^{s_{i} \mathbb{Z}}$ in $\pi_{0}$ (Symp) are different. It follows from Lemma 4.4 that this happens iff there are integral lifts $\tau_{i}, i=1,2$, of $[\omega]$ that are stabilized by different subgroups of $\pi_{0}$ (Symp). To get around this difficulty, one must reformulate the classification problem: see Gal-Kędra [3].

We next explain a very natural way to think of a splitting $s^{\mathbb{Z}}$ of $\pi_{\mathbb{Z}}$ when $\mathcal{P}_{\omega}^{\mathbb{Z}}=\mathbb{Z}$. Denote by $\tau \in H^{2}(M ; \mathbb{Z})$ some integer lift of $[\omega]$ and by $\rho: L_{\tau} \rightarrow M$ the corresponding prequantum complex line bundle. Choose a connection 1-form $\alpha$ on $L:=L_{\tau}$ with curvature $d \alpha=-\rho^{*}(\omega)$. Then $\alpha$ determines a splitting

$$
s:=s_{\alpha}
$$

as follows. By Stokes' theorem, the $\alpha$-holonomy round a nullhomologous loop $\ell$ in $M$ is multiplication by

$$
\exp \left(2 \pi i \int_{W} \omega\right), \quad \text { where } \partial W=\ell .
$$

(To see this, observe that any such $W$ can be cut open until it is a disc and so can be lifted to a 2 -disc $\widetilde{W}$ in $L$ whose boundary projects to the union of $\ell$ with some arcs that are covered twice, once in each direction.) Therefore, the holonomy of $\alpha$ defines a homomorphism $m_{\alpha}$ from the group $Z_{1}(M)$ of integral 1-cycles in $M$ to $\mathbb{R} / \mathbb{Z}$ that factors through $S H_{1}(M, \omega ; \mathbb{Z})$. Now set $s_{\alpha}[\ell]$ to be the unique element in $\pi_{\mathbb{Z}}^{-1}[\ell]$ in the kernel of $m_{\alpha}$. In other words, 
we choose $s_{\alpha}[\ell]$ so that the $\alpha$-holonomy round this loop vanishes. Two connection forms $\alpha, \alpha^{\prime}$ on $L_{\tau}$ differ by the pullback of a closed 1-form $\beta$ on $M$. Hence, if $[\ell]$ is a torsion class the element $s_{\alpha}[\ell]$ is independent of the choice of $\alpha$. Therefore, each integer lift $\tau$ of $[\omega]$ determines a family of splittings $s:=s_{\alpha}$ that give rise to the same element $C_{s} \in \operatorname{Hom}($ Tor, $\mathbb{R} / \mathbb{Z})$.

Definition 4.3. We say that a splitting $s:=s^{\mathbb{Z}}$ is $\tau$-canonical if it has the form $s_{\alpha}$ for some connection form $\alpha$ on the prequantum bundle $\rho: L_{\tau} \rightarrow M$.

Lemma 4.4. (i) Each splitting $s$ is $\tau$-canonical for a unique bundle $L_{\tau} \rightarrow M$.

(ii) Two connections forms $\alpha, \alpha^{\prime}$ on $L \rightarrow M$ give rise to the same splitting iff $\alpha-\alpha^{\prime}$ is the pullback of an exact 1-form on $M$.

(iii) Any two $\tau$-canonical splittings $s, s^{\prime}$ of $\pi_{\mathbb{Z}}$ are homotopic. Moreover, the corresponding groups $\operatorname{Ham}^{s \mathbb{Z}}$ and $\operatorname{Ham}^{s^{\prime} \mathbb{Z}}$ are conjugate by an element of Symp $_{0}$.

(iv) If $s$ is $\tau$-canonical, the image of $\pi_{0}\left(\mathrm{Ham}^{\text {s }}\right)$ in $\pi_{0}$ (Symp) is the stabilizer of $\tau$ in $\pi_{0}$ (Symp).

Proof. Statement (ii) is immediate from the construction. To prove (iii), note that two connection forms $\alpha, \alpha^{\prime}$ on $L_{\tau}$ differ by the pullback of a closed 1 -form $\beta$ on $M$. Hence the corresponding splittings $s_{\alpha}$ and $s_{\alpha^{\prime}}$ can be joined by a path of splittings that are constant on the torsion loops. The proof of Lemma 2.2 shows that this path can be lifted to an isotopy in $\mathrm{Symp}_{0}$. Therefore, $\mathrm{Ham}^{s \mathbb{Z}}$ and $\mathrm{Ham}^{s^{\prime} \mathbb{Z}}$ are conjugate as in Lemma 2.5.

To prove (i), note that the set of integer lifts $\tau$ of $[\omega]$ is a coset of the torsion subgroup Tor $H^{2}(M ; \mathbb{Z})$, while the set of splittings of $\pi_{\mathbb{Z}}$ is a coset of $\operatorname{Hom}\left(H_{1}(M ; \mathbb{Z}) ; \mathbb{R} / \mathbb{Z}\right)=H^{1}(M ; \mathbb{R} / \mathbb{Z})$. By (iii) we have set up a correspondence $\tau \mapsto s_{\alpha}$ between the set of integer lifts and the components of the space of splittings. Since these are finite sets with the same number of elements, we simply have to check that this correspondence is injective. In other words, we need to see that the isomorphism class of $L$ is determined by the set of loops that are homologically torsion and have trivial $\alpha$-holonomy (where $\alpha$ is any connection 1-form). But this is an elementary fact about complex line bundles. In fact, given bundles $L, L^{\prime}$ with connections $\alpha, \alpha^{\prime}$ that have the same curvature and have trivial holonomy round a set of loops generating $\operatorname{Tor}\left(H_{1}(M ; \mathbb{Z})\right)$, one can adjust $\alpha^{\prime}$ so that the monodromies agree on a full set of generators for $H_{1}(M ; \mathbb{Z})$ and then construct an isomorphism between the two bundles by parallel translation.

Finally note that by Lemma 4.1 the image of $\pi_{0}\left(\mathrm{Ham}^{s \mathbb{Z}}\right)$ in $\pi_{0}(\mathrm{Symp})$ is the kernel of $C_{s}$. The proof of (i) shows that if $s$ is $\tau$-canonical, then $g \in \operatorname{ker} C_{s}$ iff $g^{*}(\tau)=\tau$. This proves (iv).

We end this section by showing that when $s$ is $\tau$-canonical, the group $\mathrm{Ham}^{s \mathbb{Z}}$ has a natural geometric interpretation in terms of the bundle 
$L_{\tau} \rightarrow M$. As in Gal-Kȩdra [3], consider the group $\mathcal{G}:=\mathcal{G}_{\tau}$ of all $S^{1}$-equivariant automorphisms of the prequantum line bundle $L:=L_{\tau}$ that cover a symplectomorphism of $M$. Then there is a fibration sequence of groups and group homomorphisms

$$
\operatorname{Map}\left(M, S^{1}\right) \longrightarrow \mathcal{G}_{\tau} \stackrel{\rho}{\longrightarrow} \operatorname{Symp}_{\tau},
$$

where the elements of $\operatorname{Map}\left(M, S^{1}\right)$ act by rotations on the fibers and $\operatorname{Symp}_{\tau}$ consists of all elements in Symp that fix the given lift $\tau \in H_{2}(M ; \mathbb{Z})$ of $[\omega]$. Thus $\operatorname{Symp}_{\tau}$ is a union of components of Symp. (Note that every $g \in \operatorname{Symp}_{\tau}$ does lift to an element in $\mathcal{G}_{\tau}$. Indeed, since $g^{*}(\tau)=\tau$, there is a bundle isomorphism $\psi: L \rightarrow g^{*}(L)$. But this induces an isomorphism $\psi(x): L_{x} \rightarrow\left(g^{*} L\right)_{x}=L_{g x}$ for all $x$ and so is a lift of $g$.) Gal-Kȩdra point out that the line bundle $L_{\tau} \rightarrow M$ extends to a line bundle over the universal $M$-bundle over $B \mathcal{G}_{\tau}$. Hence, any symplectic $M$-bundle whose structural group lifts to $\mathcal{G}_{\tau}$ has an integral connection form.

Fix a unitary connection $\alpha$ on $L:=L_{\tau}$ and consider the subgroup $\mathcal{G}_{\alpha} \subset \mathcal{G}_{\tau}$ of all $S^{1}$-equivariant automorphisms of the prequantum line bundle $L$ that preserve $\alpha$. If $s$ is the splitting defined by $\alpha$, then it follows immediately from the definitions that $\mathcal{G}_{\alpha}$ projects onto the subgroup $\operatorname{Ham}^{s \mathbb{Z}}$ : cf. Kostant [8, Theorem 1.13.1]. Hence, there is a commutative diagram

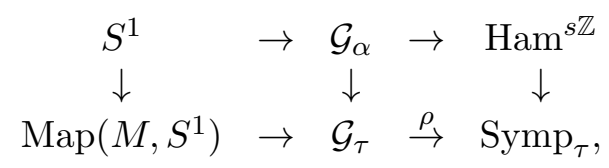

in which the top row is a central extension and the kernel $S^{1}$ of this extension is included in the bottom row as the constant maps.

Now consider the quotient group $\overline{\mathcal{G}}_{\tau}:=\mathcal{G}_{\tau} / \mathcal{N}$ where $\mathcal{N} \subset \operatorname{Map}\left(M, S^{1}\right)$ consists of all null homotopic maps $M \rightarrow S^{1}$. The fibration over $\operatorname{Symp}_{\tau}$ descends to give an extension

$$
H^{1}(M ; \mathbb{Z}) \rightarrow \overline{\mathcal{G}}_{\tau} \rightarrow \operatorname{Symp}_{\tau}
$$

We give a formula for its defining 2-cocycle in Remark 4.15 below.

Proposition 4.5. Suppose that $\mathcal{P}_{\omega}^{\mathbb{Z}}=\mathbb{Z}$. Given any $\tau$-canonical splitting $s$, the inclusion $\mathrm{Ham}^{s \mathbb{Z}} \rightarrow \mathrm{Symp}_{\tau}$ lifts to a continuous group homomorphism

$$
\iota: \operatorname{Ham}^{s \mathbb{Z}} \longrightarrow \overline{\mathcal{G}}_{\tau}
$$

that is a homotopy equivalence.

Proof. Since the induced map $\mathcal{G}_{\alpha} \rightarrow \overline{\mathcal{G}}_{\tau}$ factors through the quotient $\mathcal{G}_{\alpha} / S^{1} \cong \operatorname{Ham}^{s \mathbb{Z}}$, we have a commutative diagram

$$
\begin{aligned}
\operatorname{Ham}^{s \mathbb{Z}} & \hookrightarrow \operatorname{Symp}_{\tau} \stackrel{\widehat{F}_{\xi}}{\rightarrow} B A \\
A \rightarrow \overline{\mathcal{G}}_{\tau} & \rightarrow \operatorname{Symp}_{\tau}
\end{aligned}
$$


where $A:=H^{1}(M ; \mathbb{Z})$. Since $\iota$ induces an isomorphism on $\pi_{0}$ by construction, it suffices to check that the boundary map $\partial: \pi_{1}\left(\operatorname{Symp}_{\tau}\right) \rightarrow \pi_{0}(A)=$ $H^{1}(M ; \mathbb{Z})$ in the long exact sequence for the bottom row is given by the usual flux Flux: $\pi_{1}\left(\operatorname{Symp}_{0}\right) \rightarrow \Gamma \subset H^{1}(M ; \mathbb{Z})$. This is well known, but we include the proof for completeness.

The space $\mathcal{P}_{*}$ Symp $_{0}$ of based paths $\left(h_{t}\right)$ in Symp $_{0}$ acts on $L$ by taking $\left(x, r e^{2 \pi i \theta}\right)$ to its image under $\alpha$-parallel translation along the path $h_{t}(x)$. If $\left(h_{t}\right)$ and $\left(h_{t}^{\prime}\right)$ are two paths with endpoint $h$, then

$$
\left(h_{t}\right) \cdot\left(x, r e^{2 \pi i \theta}\right)=\lambda_{f}\left(\left(h_{t}^{\prime}\right) \cdot\left(x, r e^{2 \pi i \theta}\right)\right)=\left(h(x), r e^{2 \pi i(\theta+f(x))}\right),
$$

where $f(x)$ is the holonomy of $\alpha$ around the loop based at $h(x)$ that is formed by first going back along $h_{t}^{\prime}(x)$ and then forward along $h_{t}(x)$. When $\left(h_{t}\right)$ and $\left(h_{t}^{\prime}\right)$ are homotopic, the function $f: M \rightarrow S^{1}=\mathbb{R} / \mathbb{Z}$ is obviously null homotopic. Therefore, this gives a homomorphism

$$
\widetilde{\jmath}: \widetilde{\operatorname{Symp}} \longrightarrow \overline{\mathcal{G}}_{\tau} \text {. }
$$

Consider the loop $\phi=\left\{h_{t} \circ\left(h_{t}^{\prime}\right)^{-1}\right\}$ and observe that $f: M \rightarrow S^{1}$ is homotopic to the function $f_{\phi}$ defined by

$$
f_{\phi}(x)=\alpha \text {-holonomy round } \phi_{t}(x), \quad x \in M \text {. }
$$

Therefore, $f^{*}[d s]=f_{\phi}^{*}[d s]$, and so it suffices to prove that for any loop $\ell$ in $M$

$$
\int_{\ell} f_{\phi}^{*}(d s)=\operatorname{Flux}(\phi)[\ell] .
$$

But if $\widetilde{f}_{\ell}:[0,1] \rightarrow \mathbb{R}$ is any lift of the function $S^{1} \rightarrow S^{1}: s \mapsto f_{\phi}(\ell(s))$, equation (4.1) implies that

$$
\tilde{f}_{\ell}(1)-\tilde{f}_{\ell}(0)=\int_{[0,1] \times S^{1}} \Psi^{*} \omega=\operatorname{Flux}(\phi)[\ell],
$$

where $\Psi(t, s)=\phi_{t} \ell(s)$. The result follows.

Gal-Kȩdra give a different proof of the above result in [3, Cor. 5.14].

4.2. Lifting $\widehat{F}_{s}$. We now investigate situations in which the flux homomorphism extends to a continuous crossed homomorphism $\widetilde{F}$ with values in $H^{1}(M ; \mathbb{R}) / \Gamma$. One obvious situation when this happens is when $\Gamma=H^{1}\left(M ; \mathcal{P}_{\omega}^{\mathbb{Z}}\right)$ and $H_{1}(M ; \mathbb{Z})$ has no torsion since then we can take $\widetilde{F}=\widehat{F}_{s}^{\mathbb{Z}}$. For example, we can take $M$ to be the product of the standard $2 n$-torus with a simply connected manifold. The other cases in which we know that $\widetilde{F}$ exists have $\Gamma=0$, so that in fact $\widehat{F}_{s}$ lifts to $H^{1}(M ; \mathbb{R})$.

As observed by Kotschick-Morita [9], such a lift exists in the monotone case. In this case $\omega$ always has a closed extension - by a multiple of the first Chern class of the vertical tangent bundle - and so the flux group vanishes by Proposition 1.7. We now explain their argument. 
Kotschick and Morita consider the symplectomorphism group $\operatorname{Symp}^{\delta}$ with the discrete topology and observe that the universal $M$-bundle $M_{\mathrm{Symp}^{\delta}} \rightarrow$ $B$ Symp $^{\delta}$ has a natural flat connection (or foliation) that is transverse to the fibers. Therefore, the fiberwise symplectic form $\omega$ has a closed extension $\widetilde{\omega}$ given by the associated connection form. Suppose now that $(M, \omega)$ is monotone, i.e., that the symplectic class $[\omega] \in H^{2}(M)$ is a nonzero multiple $\lambda c_{1}(T M)$ of the first Chern class of the tangent bundle $T M$. Then $[\omega]$ has another extension $\lambda v$ where $v:=c_{1}^{\text {Vert }}$ denotes the first Chern class of the vertical tangent bundle. By construction the class $[\widetilde{\omega}]-\lambda v \in H^{2}\left(M_{\mathrm{Symp}^{\delta}}\right)$ vanishes when restricted to the fiber. Therefore, it projects to a class

$$
\left[F_{\mathrm{KM}}\right] \in E_{\infty}^{1,1}=E_{2}^{1,1}=H^{1}\left(B \operatorname{Symp}^{\delta} ; H^{1}(M ; \mathbb{R})\right)=H_{\mathrm{EM}}^{1}\left(\operatorname{Symp}^{\delta} ; H^{1}(M ; \mathbb{R})\right),
$$

where $E_{\infty}^{1,1}$ is the $(1,1)$ term in the Leray-Serre spectral sequence. Each class $[F] \in H^{1}\left(B \operatorname{Symp}^{\delta} ; \mathcal{A}\right)$ may be represented by a crossed homomorphism $F: \operatorname{Symp}^{\delta} \rightarrow \mathcal{A}$ whose restriction to the subgroup $\operatorname{Symp}_{H}^{\delta}$ that acts trivially on $\mathcal{A}$ is unique. Therefore, it makes sense to talk about the restriction of $F_{\mathrm{KM}}$ to the identity component $\mathrm{Symp}_{0}^{\delta}$. Kotschick and Morita show that this is just the usual flux homomorphism. ${ }^{9}$

We now show that the Kotschick-Morita class $\left[F_{\mathrm{KM}}\right]$ also lifts the class $\left[\widehat{F}_{s}\right]$. We do this by giving an explicit formula for a representative $\widetilde{F}_{s}$ of $\left[F_{\mathrm{KM}}\right]$. The task here is to use the monotonicity of $(M, \omega)$ to define a correction term for $\widehat{F}_{s}$ so that it takes values in $H^{1}(M ; \mathbb{R})$ rather than in $H^{1}\left(M ; \mathbb{R} / \mathcal{P}_{\omega}^{\mathbb{Z}}\right)$. If $M$ is a Riemann surface, this is quite easy: see Remark 4.7(i). However, one has to work a bit harder to derive the general formula. We shall use the notation of Section 4.1.

Theorem 4.6. Suppose that $(M, \omega)$ is monotone, i.e., that the symplectic class $[\omega] \in H^{2}(M)$ is a nonzero multiple $\lambda c_{1}$ of the first Chern class $c_{1}:=c_{1}(T M)$. Choose a splitting $s$ that is $\tau$-compatible where $\tau=\left[c_{1}\right] \in$ $H^{2}(M ; \mathbb{Z})$. Then $\widehat{F}_{s}: \operatorname{Symp}(M, \omega) \rightarrow \mathcal{A}$ lifts to a crossed homomorphism

$$
\widetilde{F}_{s}: \operatorname{Symp}(M, \omega) \rightarrow H^{1}(M ; \mathbb{R}) .
$$

Moreover, this lift represents $\left[F_{\mathrm{KM}}\right]$ in $H^{1}\left(B \operatorname{Symp}^{\delta} ; H^{1}(M ; \mathbb{R})\right)$.

Proof. Fix a basepoint $x_{0}$ in $M$ and denote by $\operatorname{Symp}_{*}\left(\operatorname{resp} . \mathrm{Ham}_{*}\right)$ the subgroup of Symp (resp. Ham) that fixes $x_{0}$. We first define $\widetilde{F}_{s}$ on the subgroup $\mathrm{Symp}_{*}$ by looking at the action of $\mathrm{Symp}_{*}$ on a certain line bundle over the universal cover of $M$. We then show that $\widetilde{F}_{s}$ lifts $\widehat{F}_{s}$ and that $\widetilde{F}_{s}$ vanishes on $\operatorname{Ham}_{*}$. It is then easy to extend $\widetilde{F}_{s}$ to a crossed homomorphism on Symp that lifts $\widehat{F}_{s}$ : see Step 4 . The proof that $\widetilde{F}_{s}$ represents the class

\footnotetext{
${ }^{9}$ As pointed out by Kȩdra-Kotschick-Morita [6], this argument works whenever $[\omega]$ extends to $H^{2}\left(M_{\text {Symp }}\right)$, which leads to an alternative proof of Corollary 1.12 (see also Remark 3.7).
} 
$F_{\mathrm{KM}}$ is based on giving an adequate description for the vertical first Chern class $v$.

Step 1: Definition of $\widetilde{F}_{s}$ on $\operatorname{Symp}_{*}$. By rescaling $[\omega]$, we may suppose that $\mathcal{P}_{\omega}^{\mathbb{Z}}=\mathbb{Z}$. Then $c_{1}(T M)=N[\omega]$, where $|N|$ is the minimal Chern number. Denote by $\widetilde{M} \rightarrow M$ the cover of $M$ associated to the homomorphism $\pi_{1}(M) \rightarrow H_{1}(M ; \mathbb{Z})$, let $L \rightarrow M$ be the complex line bundle with first Chern class $c_{1}(T M)$ and denote by $\widetilde{L} \rightarrow \widetilde{M}$ its pullback to $\widetilde{M}$. Denote by $\widetilde{x}_{0}=\left(x_{0},\left[\gamma_{0}\right]\right)$ the base point in $\widetilde{M}$ corresponding to $x_{0}$, where $\gamma_{0}$ denotes the constant path at $x_{0}$.

Fix a (unitary) connection $\alpha$ on $L$, and let $\widetilde{\alpha}$ be its pullback to $\widetilde{L}$. (Thus $d \widetilde{\alpha}$ is the pullback of $-N \omega$.) Denote by $\mathcal{G}_{\widetilde{\tau}}$ the group of $S^{1}$-equivariant diffeomorphisms of $\widetilde{\tau}$ that cover symplectomorphisms of $M$.

For each $g \in$ Symp $_{*}$, define $\widetilde{g}: \widetilde{L} \rightarrow \widetilde{L}$ as follows: the points of $\widetilde{M}$ are pairs $\left(x,\left[\gamma_{x}\right]\right)$, where $\left[\gamma_{x}\right]$ is an equivalence class of paths from $x_{0}$ to $x$ and we set

$$
\widetilde{g}\left(x,\left[\gamma_{x}\right], \theta\right):=\left(g x,\left[g \gamma_{x}\right], \theta^{\prime}\right),
$$

where $\theta^{\prime} \in \widetilde{L}_{\left(g x,\left[g \gamma_{x}\right]\right)}$ is the image of $\theta \in \widetilde{L}_{\left(x,\left[\gamma_{x}\right]\right)}$ under $\widetilde{\alpha}$-parallel translation, first back along $\gamma_{x}$ to $x_{0}$ and then forwards along $g\left(\gamma_{x}\right)$. This is independent of the choice of representative $\gamma_{x}$ for $\left[\gamma_{x}\right]$ because $g \in$ Symp $_{*}$. (Use equation (4.1) and the fact that $g$ preserves $\omega$.) Further the map $g \mapsto \widetilde{g}$ is a group homomorphism.

By construction $L$ is isomorphic to $\Lambda^{n}\left(T^{*}(M)\right)$, where $T^{*} M$ is given a complex structure compatible with $\omega$. Hence, each $g \in$ Symp lifts to a bundle automorphism $g_{L}$ of $L$ that can be chosen to preserve the Hermitian structure of $L$. Therefore the 1 -form $\widetilde{g}^{*}(\widetilde{\alpha})-\widetilde{\alpha}$ is the pullback of the 1 -form $\left(g_{L}\right)^{*}(\alpha)-\alpha$ on $M$ and hence is exact. For $g \in \operatorname{Symp}_{*}$, we define $f_{g}: \widetilde{M} \rightarrow \mathbb{R}$ to be the unique function such that $f_{g}\left(x_{0}\right)=0$ and

$$
\widetilde{g}^{*}(\widetilde{\alpha})=\widetilde{\alpha}-d f_{g}
$$

Then set

$\widetilde{F}_{s}(g)([\ell]):=\frac{1}{N}\left(f_{g}(\widetilde{\ell}(1))-f_{g}(\widetilde{\ell}(0))\right) \in \mathbb{R}, \quad g \in \operatorname{Symp}_{*}, \quad[\ell] \in H_{1}(M ; \mathbb{Z}) /$ Tor,

where $\ell$ is a based loop in $M$ representing [ $\ell]$ and the path $\widetilde{\ell}$ is its lift to $\widetilde{M}$ with initial point $\widetilde{x}_{0}$. In other words,

$$
\widetilde{F}_{s}(g)([\ell]):=\frac{1}{N}\left(m_{\widetilde{g}^{*} \widetilde{\alpha}}(\widetilde{\ell})-m_{\widetilde{\alpha}}(\widetilde{\ell})\right),
$$

where $m_{\widetilde{\beta}}(\widetilde{\ell})$ denotes the holonomy of the connection $\widetilde{\beta}$ along the path $\tilde{\ell}$. (This is only defined $\bmod \mathbb{Z}$, but the difference between two connections gives an element in $\mathbb{R}$.) It is easy to check that $\widetilde{F}_{s}(g)([\ell])$ does not depend on the chosen representative $\ell$ for $[\ell]$. This completes Step 1. 
Step 2: $\widetilde{F}_{s}$ reduces $\bmod \mathbb{Z}$ to $\widehat{F}_{s}$. It is immediate from the definitions that $\widetilde{F}_{s}: \operatorname{Symp}_{*} \rightarrow \mathbb{R}$ is a crossed homomorphism. We claim that its $\bmod \mathbb{Z}$ reduction coincides with $\widehat{F}_{s}$. Suppose first that $g[\ell]=[\ell] \in H_{1}(M ; \mathbb{Z})$. Then the lifted paths $\widetilde{\ell}$ and $\widetilde{g}(\widetilde{\ell})$ have the same endpoint and form the boundary of a 2-chain $\widetilde{C}$ in $\widetilde{M}$ that lifts a chain $C$ in $M$ with boundary $C=g(\ell)-\ell$. Hence we can calculate the $\bmod \mathbb{Z}$-reduction of $\widetilde{F}_{s}(g)([\ell])$ as follows:

$$
\begin{aligned}
\widetilde{F}_{s}(\widetilde{g})([\ell]) & =\frac{1}{N}\left(m_{\widetilde{g}^{*} \widetilde{\alpha}}(\widetilde{\ell})-m_{\widetilde{\alpha}}(\widetilde{\ell})\right) \\
& =\frac{1}{N}\left(m_{\widetilde{\alpha}}(\widetilde{g}(\widetilde{\ell}))-m_{\widetilde{\alpha}}(\widetilde{\ell})\right) \\
& =\frac{1}{N} \int_{\partial \widetilde{C}}-\widetilde{\alpha}=\int_{C} \omega \\
& =\widehat{F}_{s}(g)[\ell] \in \mathbb{R} / \mathbb{Z} .
\end{aligned}
$$

In general, $\widehat{F}_{s}(g)[\ell]$ is defined to be the area of a chain with boundary $g(\ell)-\ell^{\prime}$, where $\ell, \ell^{\prime}$ are chosen to have zero $\alpha$-holonomy (i.e., so that they project to elements in $\operatorname{Im} s$ ) and $\ell^{\prime}$ is homologous to $g(\ell)$. But now $\widetilde{g}(\widetilde{\ell})$ and $\widetilde{\ell^{\prime}}$ have the same endpoint and so

$$
\begin{aligned}
\widetilde{F}_{s}(\widetilde{g})([\ell]) & =\frac{1}{N}\left(m_{\widetilde{g}^{*} \widetilde{\alpha}}(\widetilde{\ell})-m_{\widetilde{\alpha}}\left(\widetilde{\ell}^{\prime}\right)\right), \\
& =\frac{1}{N}\left(m_{\widetilde{\alpha}}(\widetilde{g}(\widetilde{\ell}))-m_{\widetilde{\alpha}}\left(\widetilde{\ell}^{\prime}\right)\right), \\
& =\widehat{F}_{s}(g)[\ell] \in \mathbb{R} / \mathbb{Z},
\end{aligned}
$$

as before. Thus $\widetilde{F}_{s}$ lifts $\widehat{F}_{s}$ on $\operatorname{Symp}_{*}$.

Step 3: $\widetilde{F}_{s}$ vanishes on $\mathrm{Ham}_{*}$. Note first that although Ham is a perfect group, its subgroup $\mathrm{Ham}_{*}$ is not - it supports the Calabi type homomorphism $h \mapsto \int_{0}^{1} \int_{M} H_{t} \omega^{n}$, where $H_{t}$ generates $h$ and is normalized by the condition that $H_{t}\left(x_{0}\right)=0$. Hence this step does require proof.

To this end, let $h \in \mathrm{Ham}_{*}$ and choose any path $h_{t}$ from the identity to $h:=h_{1}$ such that the $\alpha$-holonomy round the loop $h_{t}\left(x_{0}\right)$ is trivial. Then $h_{t}$ lifts to a path in $\widetilde{M}$ and thence to a path $\hat{h}_{t}: \widetilde{L} \rightarrow \widetilde{L}$ given by taking the $\widetilde{\alpha}$-holonomy along $h_{t}(x)$. Let $s \mapsto \ell(s)$ be a based loop in $M$ with lift $\widetilde{\ell}$. Consider the map

$$
Y: I^{2} \longrightarrow \widetilde{M}, \quad(s, t) \longmapsto \hat{h}_{t}(\widetilde{\ell}(s)),
$$

and trivialize the bundle $Y^{*} \widetilde{L}$ by parallel translation along the horizontal line $t=0$ and the verticals $s=$ const. Then in this trivialization the map

$$
\hat{h}_{1}: \widetilde{L}_{Y([0,1] \times\{0\})} \rightarrow \widetilde{L}_{Y([0,1] \times\{1\})}
$$


has the form $\left(s, 0, r e^{2 \pi i \theta}\right) \rightarrow\left(s, 1, r e^{2 \pi i \theta}\right) \in Y^{*}(\widetilde{L})$ while the corresponding map defined by $\widetilde{h}:=\widetilde{\iota}(h)$ has the form

$$
\widetilde{h}:\left(s, 0, r e^{2 \pi i \theta}\right) \longmapsto\left(s, 1, r e^{2 \pi i(\theta-f(s))}\right),
$$

where $f(s)$ is the area of the rectangle $Y([0, s] \times[0,1])$. (Here we have used the fact that the $\alpha$-holonomy round the loop $h_{t}\left(x_{0}\right)$ is trivial so that our trivialization gives the obvious identification of the fiber $L_{x_{0}}$ over $(0,0)$ with that (also $\left.L_{x_{0}}\right)$ over $(0,1)$.) Further, because $s \mapsto Y\left(s, 0, r e^{2 \pi i \theta}\right)$ is $\widetilde{\alpha}$-parallel, the path $s \mapsto \hat{h}\left(Y\left(s, 0, r e^{2 \pi i \theta}\right)\right)$ is also $\widetilde{\alpha}$-parallel by definition of $\hat{h}$. Therefore, in this trivialization, the restriction of $\widetilde{h}^{*} \widetilde{\alpha}$ to $\widetilde{\ell}$ is $\widetilde{\alpha}-d f(s)$. Hence $\widetilde{F}_{s}(h)[\ell]=f(1)$. But because $h_{t}$ is a Hamiltonian path with no flux through $\ell$, we must have $f(1)=0$.

Step 4: End of the proof of the first statement. Given $g \in$ Symp, we now set

$$
\widetilde{F}_{s}(g):=\widetilde{F}_{s}(h g),
$$

where $h \in$ Ham is any element such that $h g \in \operatorname{Symp}_{*}$. This is independent of the choice of $h$ because $\widetilde{F}_{s}=0$ on $\operatorname{Ham}_{*}$. Moreover, $\widetilde{F}_{s}:$ Symp $\rightarrow H^{1}(M ; \mathbb{R})$ is a crossed homomorphism. It lifts $\widehat{F}_{s}$, since this also vanishes on Ham. This completes the proof of the first statement in the proposition.

Step 5: $\widetilde{F}_{s}$ represents the class $\left[F_{\mathrm{KM}}\right]$ in $H_{\mathrm{EM}}^{1}\left(\operatorname{Symp}^{\delta} ; \mathbb{R}\right)$. This is easy to see on the subgroup Ham since both crossed homomorphisms vanish there.

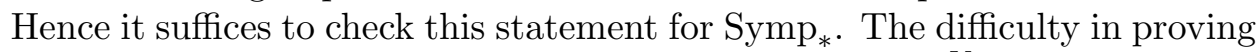
this is to find a suitable way to calculate the class $v:=c_{1}^{\text {Vert }}$.

We first consider the subgroup $S_{* H}:=\operatorname{Symp}_{* H}$ of $\operatorname{Symp}_{*}$ that acts trivially on $H_{1}(M ; \mathbb{Z})$. The $E_{1,1}^{2}$-term of the integral homology spectral sequence for the pullback of $M_{\text {Symp }^{\delta}} \rightarrow B$ Symp $^{\delta}$ to $B S_{* H}^{\delta}$ is isomorphic to the product $H_{1}\left(B S_{* H}^{\delta}\right) \otimes H_{1}(M ; \mathbb{Z})$. It is generated by cycles

$$
C_{g, \ell}:=Z_{g, \ell} \cup W_{g, \ell}
$$

where $W_{g, \ell}$ is a 2-chain in the fiber $M_{*}$ over the base point with boundary $g(\ell)-\ell$, and $Z_{g, \ell}$ is a cylinder lying over the loop in the base corresponding to $g \in \operatorname{Symp}^{\delta}$ with boundary $\ell-g(\ell)$. Since the class $[\widetilde{\omega}]$ vanishes on $Z_{g, \ell}$,

$$
\int_{C_{g, \ell}} \widetilde{\omega}=\int_{W_{g, \ell}} \omega
$$

(Note that the $\bmod \mathbb{Z}$ reduction of this class is $\widehat{F}_{s}(g)[\ell]$ as one would hope.) Since $S_{* H}$ does not act on $L$ but does act on the pullback $\widetilde{L}$, to understand the vertical Chern class $v=c_{1}^{\text {Vert }}$, we should think of $M$ as the quotient of $\widetilde{M}$ by the group $\mathrm{G}:=H_{1}(M ; \mathbb{Z})$ and consider the corresponding G-equivariant pullback line bundle $\widetilde{L}_{S^{\delta}} \rightarrow \widetilde{M}_{S^{\delta}}$. (Here we denote $S^{\delta}:=S_{* H}^{\delta}$.) Then $C_{g, \ell}$ lifts to a cycle $\widetilde{C}_{g, \ell}:=\widetilde{Z}_{g, \ell} \cup \widetilde{W}_{g, \ell}$ in $\widetilde{M}_{S^{\delta}}$. There is a trivialization of $\widetilde{L}_{S^{\delta}}$ 
over $\widetilde{Z}_{g, \ell}$ that restricts to $\widetilde{\alpha}$ over $\widetilde{\ell}$ and to $(\widetilde{g})_{*}(\widetilde{\alpha})$ over $\widetilde{g}(\widetilde{\ell})$. Extend this to any connection $\widetilde{\beta}$ of $\widetilde{L}$ over $\widetilde{W}_{g, \ell}$. Then $v\left(C_{g, \ell}\right)$ is given by integrating the curvature of this connection over $\widetilde{C}_{g, \ell}$. Since this connection is flat over $\widetilde{Z}_{g, \ell}$, the relevant part of the integral is over $\widetilde{W}_{g, \ell}$. Then because $-N \widetilde{\omega}$ pulls back to $d \widetilde{\alpha}$

$$
\begin{aligned}
\left\langle\widetilde{\omega}-\frac{1}{N} v, C_{g, \ell}\right\rangle & =\frac{1}{N} \int_{\widetilde{W}_{g, \ell}}(-d \widetilde{\alpha}+d \widetilde{\beta}) \\
& =\frac{1}{N}\left(\int_{\widetilde{g}(\widetilde{\ell})} \widetilde{\beta}-\widetilde{\alpha}-\int_{\widetilde{\ell}} \widetilde{\beta}-\widetilde{\alpha}\right) \\
& =\frac{1}{N} \int_{\widetilde{g}(\widetilde{\ell})} \widetilde{\beta}-\widetilde{\alpha}=\frac{1}{N} \int_{\widetilde{\ell}}(\widetilde{g})^{*}(\widetilde{\beta}-\widetilde{\alpha}) \\
& =\frac{1}{N} \int_{\widetilde{\ell}} \widetilde{\alpha}-(\widetilde{g})^{*} \widetilde{\alpha}=\widetilde{F}_{s}(g)[\ell] .
\end{aligned}
$$

To extend this argument to the full group $S:=\mathrm{Symp}_{*}$, we consider the 2-chains

$$
C_{g, \ell}^{\prime}=Z_{g, \ell} \cup W_{g, \ell}^{\prime},
$$

where $\ell$ is now a loop with trivial $\alpha$-holonomy, $Z_{g, \ell}$ is as before and $W_{g, \ell}^{\prime}$ is any 2-chain in $M_{*}$ with boundary $g(\ell)-\ell^{\prime}$, where $\ell^{\prime}$ is homologous to $g(\ell)$ and also has zero $\alpha$-holonomy. Thus $\partial C_{g, \ell}^{\prime}=\ell-\ell^{\prime}$, where both loops have zero $\alpha$-holonomy.

Any element in $E_{2}^{1,1}=H^{1}\left(B S^{\delta} ; H^{1}(M ; \mathbb{R})\right)$ is determined by its values on the integral 2-cycles $Z$ that are sums of chains of the form $C_{g, \ell}^{\prime}$ with chains in the fiber $M_{*}$ whose boundary consists of sums of loops with trivial $\alpha$-holonomy. Again we lift each such cycle to a cycle $\widetilde{Z}$ in $\widetilde{M}_{S^{\delta}}$ and evaluate $v$ on $\widetilde{Z}$ by integrating the curvature of a suitable connection form for the pullback of $\widetilde{L}$ to $\widetilde{Z}$. As before, we suppose that this connection equals $\widetilde{\alpha}$ on the "free" boundary arcs $\widetilde{\ell}, \widetilde{\ell^{\prime}}$. Since these have trivial $\widetilde{\alpha}$-holonomy by construction, the pieces of $\widetilde{Z}$ formed from chains in $M_{*}$ do not contribute to $[\omega]-\frac{1}{N} v$ while the contribution of $C_{g, \ell}^{\prime}$ is

$$
\left\langle\widetilde{\omega}-\frac{1}{N} v, C_{g, \ell}^{\prime}\right\rangle=\widetilde{F}_{s}(g)[\ell]
$$

as before. This completes the proof.

Remark 4.7. (i) If $(M, \omega)$ is a Riemann surface, then there is another simpler description of $\widetilde{F}_{s}$. In the notation of Section $2, \widetilde{F}_{s}(h)(\lambda)=\int_{C} \omega-$ $\frac{1}{N} c_{1}\left(\left.T M\right|_{C}\right)$, where $C$ is any integral 2-chain whose boundary represents $h_{*}(s \lambda)-s\left(h_{*} \lambda\right)$ and $c_{1}\left(\left.T M\right|_{C}\right)$ is the relative Chern number of the restriction of $T M$ to $C$ with respect to the obvious trivializations of $T M$ along the (embedded) boundary of $C$. 
(ii) The above argument used in an essential way the fact that every symplectomorphism lifts to an automorphism of $L=\Lambda^{n}\left(T^{*} M\right)$. The construction of $\widetilde{F}_{s}$ works whenever there is a line bundle $L^{\prime}$ over the universal $M$-bundle $M_{\text {Symp }} \rightarrow B$ Symp such that $c_{1}\left(L^{\prime}\right)$ restricts on the fiber to an integral lift $\tau$ of $[\omega]$, i.e., whenever there is a lift such that $\operatorname{Symp}_{\tau}=$ Symp. The second part of the argument, showing that $\widetilde{F}_{s}$ represents the corresponding element in the $E_{2}^{1,1}$-term of the spectral sequence also goes through. Hence, under these circumstances, our construction gives an explicit formula for the crossed homomorphism whose existence is established by Kędra-KotschickMorita in [6, Theorem 6].

4.3. The atoroidal case. We now consider the situation when $\omega$ vanishes on tori and/or spheres. Recall from Section 1 that the group Symp ${ }_{\pi}$ consists of all symplectomorphisms that are isotopic to an element in $\mathrm{Symp}_{*}$ that acts trivially on $\pi_{1}\left(M, x_{0}\right)$, where $x_{0}$ is the base point in $M$. This is equivalent to saying that the elements in $\operatorname{Symp}_{\pi} \cap \operatorname{Symp}_{*}$ act on $\pi_{1}\left(M, x_{0}\right)$ by inner automorphisms. We denote by

$$
\text { Symp }_{* \pi}
$$

the subgroup of $\operatorname{Symp}_{\pi} \cap \mathrm{Symp}_{*}$ that acts trivially on $\pi_{1}\left(M, x_{0}\right)$. The (disconnected) group $\mathrm{Ham}_{*}$ is a subgroup of $\mathrm{Symp}_{* \pi}$ because the evaluation map $\pi_{1}(\mathrm{Ham}) \rightarrow \pi_{1}\left(M, x_{0}\right)$ is trivial: see [14] for example. Note also that although $\Gamma$ may not vanish when $\omega=0$ on $\pi_{2}(M)$, the Flux homomorphism vanishes on loops in $\operatorname{Symp}_{* \pi} \cap \mathrm{Symp}_{0}$ since its value is then given by evaluating $[\omega]$ on 2-spheres. Therefore, in this case Flux is well defined as a homomorphism $\operatorname{Symp}_{* \pi} \cap \operatorname{Symp}_{0} \rightarrow H^{1}(M ; \mathbb{R})$.

Lemma 4.8. If $\omega=0$ on spheres, then Flux extends to a homomorphism $F_{\pi}: \operatorname{Symp}_{* \pi} \rightarrow H^{1}(M ; \mathbb{R})$.

Proof. Denote by $S \pi_{1}(M)$ the group formed by equivalence classes $\langle\gamma\rangle$ of based loops in $\left(M, x_{0}\right)$ where two loops $\gamma_{0}, \gamma_{1}$ are equivalent iff they may be joined by a based homotopy $\gamma_{t}$ of zero symplectic area, i.e.,

$$
\int_{C} \psi^{*} \omega=0, \psi: C=S^{1} \times[0,1] \longrightarrow M,(s, t) \longmapsto \gamma_{t}(s) .
$$

Because $\omega$ vanishes on spheres, the symplectic area of a based homotopy between two homotopic loops $\gamma_{0}, \gamma_{1}$ is independent of the choice of homotopy. As in Lemma 2.1, it follows readily that there is an exact sequence of groups

$$
0 \rightarrow \mathbb{R} \rightarrow S \pi_{1}(M) \stackrel{\pi}{\rightarrow} \pi_{1}(M) \rightarrow\{1\} .
$$

We now define a map $s: \pi_{1}(M) \rightarrow S \pi_{1}(M)$ such that $\pi \circ s=$ id. Since $\omega$ vanishes on spheres, it lifts to an exact form $\widetilde{\omega}$ on the universal cover $\widetilde{M}$ of $M$. Choose a base point $\widetilde{x}_{0}$ of $\widetilde{M}$ that projects to $x_{0}$ and choose a 1 -form $\beta$ on $\widetilde{M}$ such that $d \beta=\widetilde{\omega}$. Then define $s:=s_{\beta}$ by setting $s_{\beta}([\gamma])=\langle\bar{\gamma}\rangle$, 
where the based loop $\bar{\gamma}$ represents $[\gamma]$ and is such that $\beta$ integrates to zero over the unique lift of $\bar{\gamma}$ to a path in $\widetilde{M}$ starting at $\widetilde{x}_{0}$. Note that $s:=s_{\beta}$ need not be a group homomorphism because $\beta$ is not invariant under deck transformations.

For each $g \in \operatorname{Symp}_{* \pi}$, define a function

$$
F_{\pi}(g): \pi_{1}(M) \longrightarrow \mathbb{R}
$$

by setting $F_{\pi}(g)([\gamma])$ equal to the symplectic area of a based homotopy joining $\overline{g(\gamma)}=\bar{\gamma}$ to $g \bar{\gamma}$. We claim that the map $F_{\pi}(g): \pi_{1}(M) \rightarrow \mathbb{R}$ is a homomorphism for each $g \in \operatorname{Symp}_{* \pi}$ and so defines an element $F_{\pi}(g)$ in $H^{1}(M ; \mathbb{R})$. To see this, define $A(\gamma, \delta)$ to be the $\omega$-area of any homotopy from $\bar{\gamma} * \bar{\delta}$ to $\overline{\gamma * \delta}$, where $\gamma, \delta \in \pi_{1}(M)$ and $\bar{\gamma} * \bar{\delta}$ denotes the loop that first goes round $\bar{\gamma}$ and then $\bar{\delta}$. Now $F_{\pi}(g)([\gamma * \delta])$ is the area of any homotopy from $\overline{g(\gamma * \delta)}$ to $g(\overline{\gamma * \delta})$. Consider the three part homotopy that goes first from $\overline{g(\gamma * \delta)}$ to $\overline{g \gamma} * \overline{g \delta}$, then to $g \bar{\gamma} * g \bar{\delta}$ and finally to $g(\overline{\gamma * \delta})$. The first of these homotopies has area $-A(g \gamma, g \delta)=-A(\gamma, \delta)$ since $g$ acts trivially on $\pi_{1}$. Therefore, this cancels out the area of the third homotopy. The middle homotopy can be chosen to be the juxtaposition of the homotopies used to define $F_{\pi}(g)[\gamma]$ and $F_{\pi}(g)[\delta]$ and so has area equal to their sum. Thus $F_{\pi}(g)([\gamma * \delta])=F_{\pi}(g)([\gamma])+F_{\pi}(g)([\delta])$.

One now shows that $F_{\pi}: \operatorname{Symp}_{* \pi} \rightarrow H^{1}(M ; \mathbb{R})$ is a homomorphism as in Proposition 2.3.

Corollary 4.9. If $\omega$ vanishes on tori, then $F_{\pi}$ extends to a homomorphism $\operatorname{Symp}_{\pi} \rightarrow H^{1}(M ; \mathbb{R})$.

Proof. Since each $g \in \operatorname{Symp}_{\pi}$ may be joined to a point $g h_{1} \in \operatorname{Symp}_{* \pi}$ by a Hamiltonian path $h_{t} \in$ Ham, we extend $F_{\pi}$ by setting

$$
F_{\pi}(g):=F_{\pi}(g h), \quad g \in \mathrm{Symp}_{\pi}, \quad g h \in \mathrm{Symp}_{* \pi}, \quad h \in \mathrm{Ham} .
$$

This is independent of the choice of $h_{t} \in$ Ham because $\omega=0$ on tori.

The above proof was written using geometric language to imitate the definition of $\widehat{F}_{s}$. However, when $g \in \operatorname{Symp}_{* \pi}$, it is perhaps more illuminating to write $F_{\pi}(g) \in \operatorname{Map}\left(\pi_{1}(M), \mathbb{R}\right)$ in the form

$$
F_{\pi}(g)(\gamma)=\kappa_{g}(\hat{\gamma}), \quad \gamma \in \pi_{1}\left(M, x_{0}\right), \quad g \in \operatorname{Symp}_{* \pi}
$$

where $\kappa_{g}: \widetilde{M} \rightarrow \mathbb{R}$ is the unique function such that $\widetilde{g}^{*} \beta=\beta+d \kappa_{g}$ and $\kappa_{g}\left(\widetilde{x}_{0}\right)=0$. Here $\widetilde{g}$ denotes the lift of $g$ to the universal cover $\widetilde{M}$ that fixes the base point $\widetilde{x}_{0}$, and $\hat{\gamma} \in \pi^{-1}\left(x_{0}\right) \subset \widetilde{M}$ is the endpoint of the lift of any representative of $\gamma \in \pi_{1}(M)$. Hence

$$
\kappa_{g h}=\kappa_{h}+\widetilde{h}^{*} \kappa_{h}, \quad F_{\pi}(g h)=F_{\pi}(h)+h^{*} F_{\pi}(g) .
$$

The above identities hold for all $g \in \mathrm{Symp}_{*}$. However, when $g \in \mathrm{Symp}_{* \pi}$, we saw above that the map $F(g)$ is a group homomorphism $\pi_{1}(M) \rightarrow \mathbb{R}$ 
for each $g$. In general this is not true. To explain the algebra, denote by $\psi_{\gamma}: \widetilde{M} \rightarrow \widetilde{M}$ the deck transformation corresponding to $\gamma \in \pi_{1}(M)$ and define the function $f_{\gamma}: \widetilde{M} \rightarrow \mathbb{R}$ by

$$
\left(\psi_{\gamma}\right)^{*} \beta=\beta+d f_{\gamma}, \quad f_{\gamma}\left(\widetilde{x}_{0}\right)=0 .
$$

Then

$$
f_{\gamma \delta}-f_{\delta}-\left(\psi_{\delta}\right)^{*} f_{\gamma}=f_{\gamma}(\hat{\delta}),
$$

where we think of the right hand side as a constant function on $M$. Therefore,

$$
A(\gamma, \delta)=\int_{\psi_{\gamma} \bar{\delta}} \beta=\int_{\bar{\delta}}\left(\psi_{\gamma}\right)^{*}(\beta)=\int_{\bar{\delta}} d f_{\gamma}=f_{\gamma}(\hat{\delta}) .
$$

Hence $A: \pi_{1} \times \pi_{1} \rightarrow \mathbb{R}$ satisfies the cocycle condition

$$
A(\delta, \varepsilon)-A(\gamma \delta, \varepsilon)+A(\gamma, \delta \varepsilon)-A(\gamma, \delta)=0 .
$$

(When checking this, it is useful to think of $A(\gamma, \delta)=\left(\psi_{\varepsilon}\right)^{*} A(\gamma, \delta)$ as a constant function on $\widetilde{M}$ and to use (4.6).) Then the identity $\widetilde{g} \circ \psi_{\gamma}=\psi_{g \gamma} \circ \widetilde{g}$ implies that

$$
f_{g \gamma} \circ \widetilde{g}-f_{\gamma}=\kappa_{g} \circ \psi_{\gamma}-\kappa_{g}-\kappa_{g}(\hat{\gamma})
$$

It follows that

$$
F_{\pi}(g)(\gamma \delta)-F_{\pi}(g)(\gamma)-F_{\pi}(g)(\delta)=f_{g \gamma}(\hat{g} \delta)-f_{\gamma}(\hat{\delta})=g^{*} A(\gamma, \delta)-A(\gamma, \delta) .
$$

Therefore, if we modify (4.5) by setting

$$
F_{\pi}(g, \gamma)(\delta):=\kappa_{g}(\hat{\gamma})+g^{*} A(\gamma, \delta)-A(\gamma, \delta),
$$

we find that

$$
F_{\pi}(g, 1)(\gamma \delta)=F_{\pi}(g, 1)(\gamma)+F_{\pi}(g, \gamma)(\delta) .
$$

This discussion applies when $\omega$ vanishes on $\pi_{2}(M)$. If $\omega$ also vanishes on tori, ${ }^{10}$ then $\Gamma=0$ and $F_{\pi}(g)=\operatorname{Flux}(g)$ for $g \in \operatorname{Symp}_{* 0}$, so that we can extend $F_{\pi}$ to the whole group Symp by equation (4.4). Hence we have shown:

Proposition 4.10. Suppose that $\omega$ vanishes on tori. Then Flux extends to a crossed homomorphism

$$
F_{\pi}: \operatorname{Symp} \rightarrow \operatorname{Map}\left(\pi_{1}(M) ; \mathbb{R}\right),
$$

such that $F_{\pi}(g) \in \operatorname{Hom}\left(\pi_{1}(M) ; \mathbb{R}\right)$ for $g \in \operatorname{Symp}_{\pi}$.

\footnotetext{
${ }^{10}$ If $\omega$ does not vanish on tori, we still have $F(g)=\operatorname{Flux}(g) \bmod \Gamma$, provided that the evaluation map $\pi_{1}$ Symp $_{0} \rightarrow \pi_{1}(M)$ is surjective. So in this case, we also get an extension of Flux.
} 
Because of the rather complicated algebraic structure of the map $F_{\pi}(g)$, when $g$ acts nontrivially on $\pi_{1}$, the kernel of $F_{\pi}$ will not in general intersect every component of Symp. Note also that if $\omega$ lifts to an exact form on the Abelian cover of $M$ given by the homomorphism $\pi_{1}(M) \rightarrow H_{1}(M ; \mathbb{Z}) /$ Tor, one can play the same game there, defining $F_{H}$ and $A$ by equations (4.5) and (4.7). Thus:

Corollary 4.11. Suppose that $[\omega] \in\left(H^{1}(M ; \mathbb{R})\right)^{2} \subset H^{2}(M ; \mathbb{R})$ and that $\omega=0$ on tori. Then Flux extends to a crossed homomorphism

$$
F_{H}: \operatorname{Symp}_{H} \rightarrow H^{1}(M ; \mathbb{R}),
$$

where $\operatorname{Symp}_{H}$ is the subgroup of Symp that acts trivially on $H_{1}(M ; \mathbb{R})$.

4.4. c-Hamiltonian bundles and covering groups. Suppose now that $(M, a)$ is a $c$-symplectic manifold, that is, a closed oriented $2 n$-dimensional manifold equipped with a class $a \in H^{2}(M ; \mathbb{R})$ such that $a^{n}>0$. Then the analogue of the symplectic group Symp is the group Diff ${ }^{a}$ of all diffeomorphisms whose action on $H^{2}(M ; \mathbb{R})$ preserves $a$. Thus its identity component is the full group $\operatorname{Diff}_{0}$. Since this is a simple group, there is no subgroup corresponding to the Hamiltonian group. However, as noted in Kȩdra-McDuff [7], there is a covering group, which may be defined as follows.

Consider the $a$-Flux homomorphism Flux ${ }^{a}: \pi_{1}\left(\operatorname{Diff}_{0}\right) \rightarrow H^{1}(M ; \mathbb{R})$, whose value on a loop $\lambda$ in $\operatorname{Diff}_{0}(M)$ is the cohomology class $\operatorname{Flux}^{a}(\lambda)$, where, for a 1-cycle $\gamma$ in $M, \operatorname{Flux}^{a}(\lambda)(\gamma)$ is given by evaluating $a$ on the 2-cycle defined by the map $\mathbb{T}^{2} \rightarrow M,(s, t) \mapsto \lambda(t)(\gamma(s))$. Set ${ }^{11}$

$$
\Gamma_{a}:=\operatorname{Im}\left(\operatorname{Flux}_{a}\right) \subset H^{1}\left(M ; \mathcal{P}_{a}^{\mathbb{Z}}\right) .
$$

We define the $c$-Hamiltonian group HDiffa to be the corresponding covering space of $\operatorname{Diff}_{0}:=\operatorname{Diff}_{0}(M)$. Thus there is a group extension:

$$
\Gamma_{a} \rightarrow \operatorname{HDiff}_{0}^{a} \rightarrow \operatorname{Diff}_{0} .
$$

A similar construction in the symplectic case gives a group extension

$$
\Gamma \rightarrow \mathcal{H}_{0} \rightarrow \text { Symp }_{0}
$$

such that the inclusion Ham $\hookrightarrow$ Symp $_{0}$ has a natural lift to a homotopy equivalence $\iota:$ Ham $\rightarrow \mathcal{H}_{0}$. To see this, note that if $h \in \mathrm{Ham}$, the element in $\mathcal{H}_{0}$ represented by the pair $(h, \gamma)$, where $\gamma$ is any path in $\mathcal{H}$ from the identity to $h$, is independent of the choice of $\gamma$ because the difference between two such paths lies in the kernel of Flux ${ }^{[\omega]}$.

Here are some analogs for Diff ${ }^{a}$ of the questions considered earlier.

Question 4.12. Suppose that $M \rightarrow P \rightarrow B$ is a bundle with structural group Diff ${ }^{a}$. When does the class $a \in H^{2}(M)$ extend to a class $\widetilde{a} \in H^{2}(P)$ ?

\footnotetext{
${ }^{11}$ By McDuff [13, Theorem 6], $\Gamma_{a}$ need not be discrete.
} 
Question 4.13. When is there a group extension

$$
\Gamma_{a} \longrightarrow \operatorname{HDiff}^{a} \longrightarrow \operatorname{Diff}^{a}
$$

that restricts over Diff $_{0}$ to the extension $\Gamma_{a} \rightarrow \operatorname{HDiff}_{0}^{a} \rightarrow \operatorname{Diff}_{0}$ ?

Neither question has an obvious answer. It is also not clear what relation they have to each other. For example, consider the case when $\Gamma_{a}:=\operatorname{Im}\left(\right.$ Flux $\left.^{a}\right)=0$. Then the cover is trivial and so always extends, but a may not always extend. We shall see below that Question 4.13 is, at least to some extent, analogous to Question 1.5 which asks when Flux extends. However, in the present situation, there is so far no analog of Theorem 1.10 which shows the close relation between the existence of an extension of Flux and the obstruction cocycle $\mathcal{O}^{M}$.

The cocycle $\varepsilon_{0} \in H_{\mathrm{EM}}^{2}\left(\operatorname{Diff}_{0}^{a} ; \Gamma_{a}\right)$ that determines the extension $\Gamma_{a} \rightarrow$ HDiff $_{0}^{a} \rightarrow$ Diff $_{0}$ is defined as follows. For each $g \in$ Diff $_{0}$, choose a path $\gamma_{g}$ from the identity element to $g$ and then define $\varepsilon_{0}(g, h)$ to be the value of Flux $^{a}$ on the loop formed by going along $\gamma_{h}$ to $h$, then along $\gamma_{g} h$ to $g h$ and then back along $\gamma_{g h}$. In the symplectic case, $\varepsilon_{0}(g, h)$ can be defined as the sum:

$$
\varepsilon_{0}(g, h)=\operatorname{Flux}\left(\gamma_{h}\right)+\operatorname{Flux}\left(\gamma_{g}\right)-\operatorname{Flux}\left(\gamma_{g h}\right) .
$$

The next result shows that $\varepsilon_{0}$ extends to a cocycle $\varepsilon$ on Symp when Flux extends.

Proposition 4.14. Suppose that Flux extends to a crossed homomorphism $\widetilde{F}:$ Symp $\rightarrow H_{\mathbb{R}} / \Gamma$, and set $\mathcal{H}_{\widetilde{F}}:=\operatorname{ker} \widetilde{F}$. For each $g \in$ Symp, pick an element $x_{g} \in \mathcal{H}_{\widetilde{F}}$ that is isotopic to $g$ and choose a path $\gamma_{g}$ in Symp $_{0}$ from the identity to $g x_{g}^{-1}$. Then:

(i) The formula

$$
\varepsilon(g, h)=\operatorname{Flux}\left(\gamma_{h}\right)+h^{*} \operatorname{Flux}\left(\gamma_{g}\right)-\operatorname{Flux}\left(\gamma_{g h}\right),
$$

defines an element $[\varepsilon] \in H_{\mathrm{EM}}^{2}(\mathrm{Symp} ; \Gamma)$ that is independent of choices.

(ii) The inclusion $\mathcal{H}_{\widetilde{F}} \rightarrow$ Symp lifts to a homotopy equivalence between $\mathcal{H}_{\widetilde{F}}$ and the covering group $\widetilde{\mathcal{G}}$ of Symp defined by $\varepsilon$.

Proof. To check (i), first observe that $x_{g}$ is determined modulo an element in Ham and so the element $\operatorname{Flux}\left(\gamma_{h}\right)$ is independent of choices modulo an element $c_{h} \in \Gamma$ that depends on the choice of path $\gamma_{h}$. The three paths $\gamma_{h}, \gamma_{g} h x_{h}^{-1},-\gamma_{g h}$ no longer make up a triangle; to close them up into a loop one needs to add a path from $g x_{g}^{-1} h x_{h}^{-1}$ to $g h x_{g h}^{-1}$. But there are elements $k, k^{\prime} \in$ Ham such that

$$
g x_{g}^{-1} h x_{h}^{-1}=g h x_{g}^{-1} k x_{h}^{-1}=g h x_{g h}^{-1} k^{\prime}
$$


and so we can choose this path to lie in Ham. It follows that formula (4.8) does define an element $\varepsilon(g, h) \in \Gamma$. The cocycle condition

$$
\delta \varepsilon(g, h, k)=\varepsilon(h, k)-\varepsilon(g, h k)+\varepsilon(g h, k)-k^{*} \varepsilon(g, h)
$$

follows by an easy calculation. Moreover, different choices of the paths $\gamma_{h}$ change $\varepsilon$ by a coboundary. This proves (i).

To prove (ii), observe that the extension $\widetilde{\mathcal{G}}$ defined by $\varepsilon$ has elements $(g, a) \in \operatorname{Symp} \times \Gamma$ where

$$
(g, a)(h, b)=\left(g h, h^{*} a+b+\varepsilon(g, h)\right) .
$$

Now consider the map $\tilde{f}: \widetilde{\mathcal{G}} \rightarrow H_{\mathbb{R}}$ given by

$$
\tilde{f}(g, a)=\widetilde{\operatorname{Flux}}\left(\gamma_{g}\right)-a \in H^{1}(M ; \mathbb{R}),
$$

where $\widetilde{\operatorname{Flux}}\left(\gamma_{g}\right)$ is the flux along the path $\gamma_{g}$ in the universal cover $\widetilde{\text { Symp. }}$. It follows immediately from the definitions that this is a crossed homomorphism with kernel $\widetilde{\mathcal{H}}$, say. Thus there is a commutative diagram with exact rows and columns

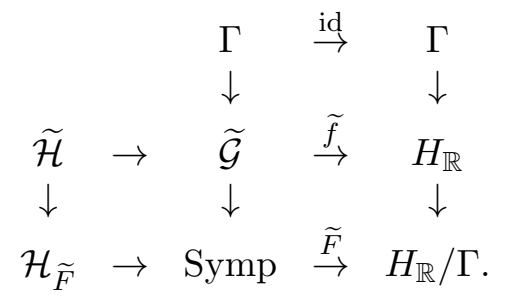

Because $H_{\mathbb{R}}$ is contractible, the 5 -lemma implies that the map $\widetilde{\mathcal{G}} \simeq \widetilde{\mathcal{H}} \rightarrow \mathcal{H}_{\mathcal{F}}$ is a homotopy equivalence.

Remark 4.15. (i) Suppose that $\mathcal{P}_{\omega}^{\mathbb{Z}}=\mathbb{Z}$, and repeat the above argument using the crossed homomorphism $\widehat{F}_{s}^{\mathbb{Z}}$, where $s$ is $\tau$-compatible. By Proposition 4.5, $\mathrm{Ham}^{s \mathbb{Z}}$ maps onto $\pi_{0}\left(\mathrm{Symp}_{\tau}\right)$. Hence $\mathrm{Ham}^{s \mathbb{Z}}$ is homotopy equivalent to the covering group of $\operatorname{Symp}_{\tau}$ defined by the cocycle $\varepsilon_{s} \in H_{c \mathrm{EM}}^{2}\left(\mathrm{Symp}_{\tau} ; H_{\mathbb{Z}}\right)$ of (4.8). It follows from Proposition 4.5 that this covering group may be identified with $\overline{\mathcal{G}}_{\tau}$.

(ii) If $F: G \rightarrow \mathcal{A} / \Lambda$ is a continuous crossed homomorphism, then there is an associated (possibly discontinuous) extension cocycle $\varepsilon_{F} \in H_{E M}^{2}(G ; \Lambda)$ ) defined by $\varepsilon_{F}(g, h)=h^{*} f(g)+f(h)-f(g h)$, where $f: G \rightarrow \mathcal{A}$ is any lift of $F$. It is not hard to check that the corresponding extension of $G$ coincides with the covering group $\widetilde{G}$ constructed from $F$ in Remark 1.21(iii).

Observe finally that we can relax Question 4.13 by asking for an extension of Diff ${ }^{a}$ by the group $H^{1}\left(M ; \mathcal{P}_{a}^{\mathbb{Z}}\right)$ rather than by $\Gamma$. If $a$ is a primitive integral class (i.e., $\mathcal{P}_{a}^{\mathbb{Z}}=\mathbb{Z}$ ), there are natural candidates for such an extension just as in the symplectic case. Moreover, the existence of these groups should have some bearing on Question 4.12. Since these questions are very similar 
to those already discussed in connection with the group $\mathrm{Ham}^{s \mathbb{Z}}$, we shall not pursue them further here.

\section{References}

[1] A. Banyaga, Sur la structure du groupe des difféomorphismes qui préservent une forme symplectique. Communicationes Mathematicae Helveticae 53 (1978), 174-227.

[2] R. Bott and L. Tu, Differential forms in algebraic topology. Graduate Texts in Mathematics, 82, Springer-Verlag, Berlin, 1982.

[3] S. Gal and J. Kȩdra, Symplectic configurations, SG/0504100, to appear in International Math. Reaearch Notes.

[4] M. Gotay, R. Lashof, J. Sniatycki and A. Weinstein, Closed forms on symplectic fiber bundles. Commentarii Mathematicae Helveticae 58 (1983), 617-621.

[5] P. Iglesias, Y. Karshon and M. Zadke, Orbifolds as Diffeologies, DG/0501093.

[6] J. Kędra, D. Kotschick and S. Morita, Crossed Flux homomorphisms and vanishing theorems for Flux groups, AT/0503230, to appear in Geometric and Functional Analysis.

[7] J. Kȩdra and D. McDuff, Homotopy properties of Hamiltonian group actions, Geometry and Topology 9 (2005), 121-162.

[8] B. Kostant, Quantization and Unitary Representations, Springer Lecture Notes, 170, 1970, 87-208.

[9] D. Kotschick and S. Morita, Signatures of foliated surface bundles and the symplectomorphism groups of surfaces, Topology, 44 (2005), 131-149.

[10] D. Kotschick and S. Morita, Characteristic classes of foliated symplectic surface bundles with area-preserving holonomy, SG/0409214, to appear in Journ. Diff. Geom.

[11] F. Lalonde and D. McDuff, Symplectic Structures on Fiber Bundles, Topology 42 (2003), 309-347 [Erratum 44 (2005), 1301-1303] SG/0010275.

[12] F. Lalonde, D. McDuff and L. Polterovich, Topological Rigidity of Hamiltonian Loops and Quantum Homology, Invent. Math. 135 (1999), 369-385.

[13] D. McDuff, Symplectic Diffeomorphisms and the Flux Homomorphism, Invent. Math. 77 (1984), 353-366.

[14] D. McDuff, A survey of topological properties of groups of symplectomorphisms, in Topology, Geometry and Quantum Field Theory, U.L. Tillmann, (ed.) (Proceedings of 2002 Symposium in honor of G.B. Segal), Cambridge University Press, 2004, $173-193$.

[15] D. McDuff and D. Salamon, Introduction to Symplectic Topology, 2nd edition, OUP, Oxford, UK, 1998.

[16] D. McDuff and D. Salamon, J-holomorphic Curves and Symplectic Topology, Colloquium Publications, American Math Society, 2004.

[17] K. Ono, Floer-Novikov cohomology and the flux conjecture, 2004.

[18] L. Polterovich, Growth of maps, distortion in groups and symplectic geometry, Invent Math. 150 (2002), 655-686.

[19] G. Segal, Classifying spaces related to foliations, Topology 17 (1978), 367-382.

[20] P. Seidel, Lectures on four-dimensional Dehn twists, SG/0309012.

[21] A. Tsemo, Gerbes, 2-gerbes and symplectic fibrations, DG/0504274. 
Department of Mathematics

STONY Brook UnIVERSITY

STONY BROOK, NY 11794-3651

E-mail address: dusa@math.sunysb.edu

Received 01/10/2005, revised 05/01/2005 and 11/08/2005. The author thanks Swiatoslav Gal, Jarek Kȩdra, Jack Milnor, Leonid Polterovich and Zhigang Han for useful discussions. She also thanks Gal and Kȩdra for showing her early drafts of their paper [3] and making various helpful comments about earlier versions of this paper. In particular, they helped streamline the proof of Lemma 3.4 and correct some details in Proposition 3.3. Finally, she wishes to thank the referees for helping to improve the presentation of the results discussed here. Partly supported by NSF grant DMS-0305939. 